DANIEL GRAÇA FATORI DE SÁ

\title{
Custo econômico e social do transtorno obsessivo- compulsivo e outros transtornos mentais na infância
}

Tese apresentada à Faculdade de Medicina da Universidade de

São Paulo para obtenção do título de Doutor em Ciências

Programa de Psiquiatria

Orientador: Prof. Dr. Euripedes Constantino Miguel Filho

(Versão corrigida. Resolução CoPGr 6018/11, de 1 de novembro de 2011. A versão original está disponível na Biblioteca da FMUSP)

São Paulo 


\section{Dados Internacionais de Catalogação na Publicação (CIP)}

Preparada pela Biblioteca da

Faculdade de Medicina da Universidade de São Paulo

Creprodução autorizada pelo autor

\section{Sá, Daniel Graça Fatori de}

Custo econômico e social do transtorno obsessivo-compulsivo e outros transtornos mentais na infância / Daniel Graça Fatori de Sá. -- São Paulo, 2016.

Tese(doutorado)--Faculdade de Medicina da Universidade de São Paulo.

Programa de Psiquiatria.

Orientador: Eurípedes Constantino Miguel Filho

Descritores: 1.Custos e análise de custo 2.Transtornos mentais diagnosticados na infância 3.Transtorno obsessivo-compulsivo 4.Epidemiologia 5.Estudos transversais

USP/FM/DBD-127/16 
Às crianças e adolescentes de todas as regiões do Brasil vivendo em condições adversas. 


\section{Agradecimentos}

Ao Prof. Dr. Euripedes Constantino Miguel, orientador desta tese, que desde o início do processo me deu plena confiança e suporte para realizar o trabalho da melhor forma possível.

À Dra. Ana Soledade Graeff-Martins, que me deu oportunidade de iniciar minha jornada no campo da pesquisa e clínica da saúde mental infantil lá em 2006. Desde então, tem servido de modelo permanente de ética, seriedade e compromisso com a pesquisa científica.

Aos membros do Instituto Nacional de Psiquiatria do Desenvolvimento (INPD) pela oportunidade e honra de trabalhar com os dados do estudo High Risk Cohort Study (HRC).

Aos professores do Departamento de Medicina Preventiva, Profa. Dra. Maria Fernanda Tourinho Peres, Profa. Dra. Patrícia Coelho de Soárez, Profa. Dra. Hillegonda Maria Dutilh Novaes, Prof. Dr. Fernando Mussa Abujamra Aith, Profa. Dra. Ana Luiza D Avila Viana, Prof. Dr. Mário César Scheffer, por oferecem disciplinas compromissadas com o conhecimento e metodologia científica.

Aos professores membros da banca examinadora de qualificação, Profa. Dra. Patrícia Coelho de Soárez, Prof. Dr. Guilherme Polanczyk, Profa. Dra. Roseli Gedanke Shavitt, por terem contribuído em pontos fundamentais no desenvolvimento e aprimoramento da tese aqui apresentada. 
Aos professores membros da banca examinadora da defesa da presente tese, Prof. Dr. Naercio Aquino Menezes Filho, Profa. Dra. Luciane Nascimento Cruz, Profa. Dra. Patrícia Coelho de Soárez, Prof. Dr. Guilherme Polanczyk, pela leitura cuidadosa da tese e por proporcionarem discussões importantes para o aprimoramento do trabalho.

Aos professores, Dr. James Leckman e Dr. Robert Rosenheck, pela oportunidade de visitar a Yale University e poder discutir dados da presente tese e projetos futuros.

Ao Prof. Dr. Alex Itria por oferecer o suporte necessário para adentrar o denso campo da avaliação econômica em saúde.

Ao Dr. Wagner Ribeiro, pela amizade e parceria ao longo dos anos, pelas discussões constantes sobre o fazer científico, por demonstrar que ciência pode e deve ser muito mais que a produção de artigos.

À Renata Silva, pelo suporte, companheirismo e carinho, assim pela cuidadosa revisão do texto da tese.

Ao Santander, pela Bolsa Mobilidade Internacional (2014) por financiar meu breve intercâmbio na Yale University.

À Fundação de Amparo a Pesquisa do Estado de São Paulo (FAPESP) por ter proporcionado minha bolsa de doutorado que contribuiu a autonomia necessária para realizar minha formação acadêmica. À FAPESP e ao Conselho Nacional de 
Desenvolvimento Científico e Tecnológico (CNPq) pelo financiamento do INPD e HRC.

Às famílias do município de São Paulo e Porto Alegre que participaram do estudo epidemiológico que compõe a base dessa tese, por terem contribuído pacientemente na coleta de dados. 


\section{Normalização adotada}

Esta dissertação ou tese está de acordo com as seguintes normas, em vigor no momento desta publicação:

Referências: adaptado de International Committee of Medical Journals Editors (Vancouver).

Universidade de São Paulo. Faculdade de Medicina. Divisão de Biblioteca e Documentação. Guia de apresentação de dissertações, teses e monografias. Elaborado por Anneliese Carneiro da Cunha, Maria Julia de A. L. Freddi, Maria F. Crestana, Marinalva de Souza Aragão, Suely Campos Cardoso, Valéria Vilhena. 3a ed. São Paulo: Divisão de Biblioteca e Documentação; 2011.

Abreviaturas dos títulos dos periódicos de acordo com List of Journals Indexed in Index Medicus. 


\section{SUMÁRIO}

LISTA DE SIGLAS E ABREVIATURAS

LISTA DE TABELAS

LISTA DE FIGURAS

RESUMO

ABSTRACT

1. INTRODUÇÃO

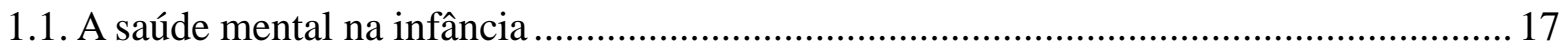

1.2. O transtorno obsessivo-compulsivo na infância........................................................ 21

1.3. O custo dos transtornos mentais: avaliação econômica em saúde mental....................... 24

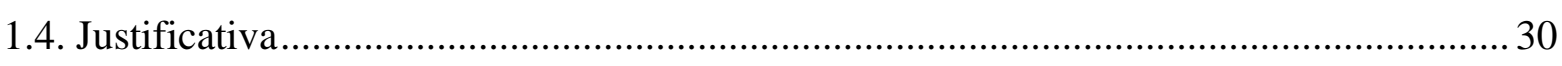

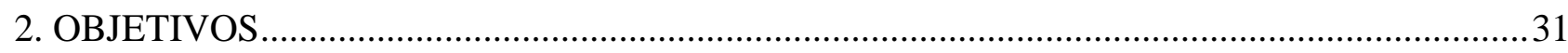

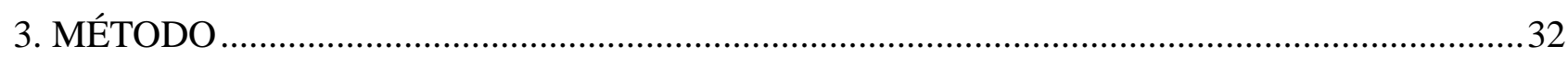

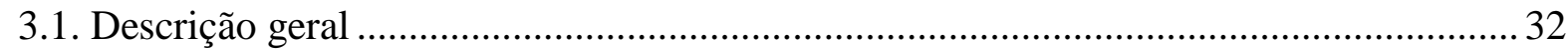

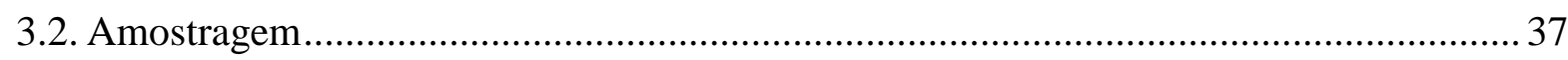

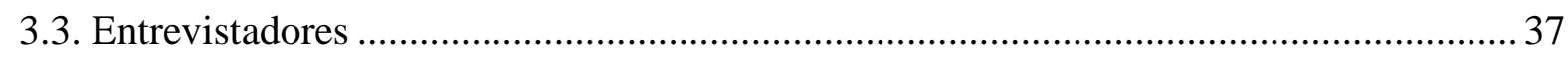

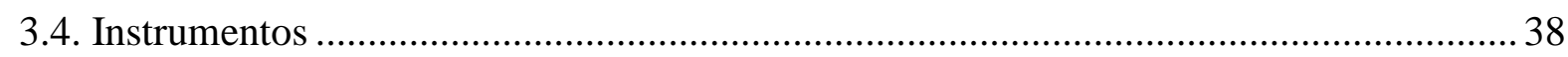

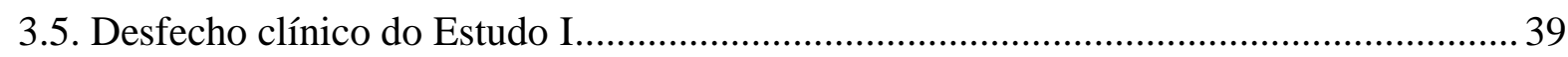

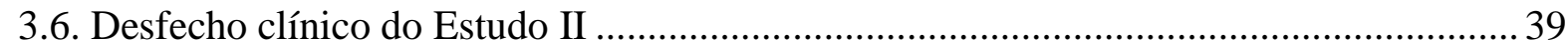

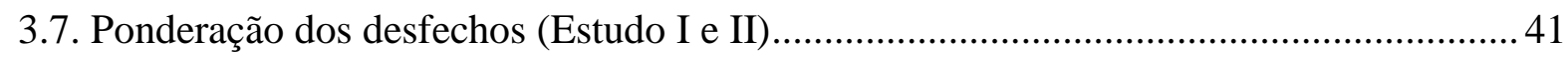

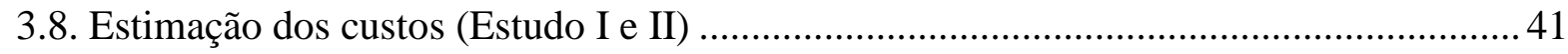

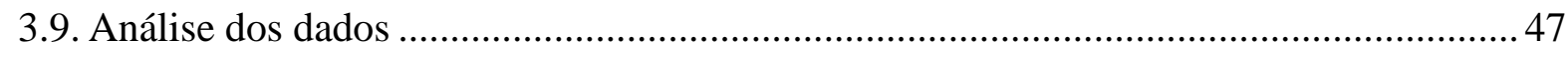

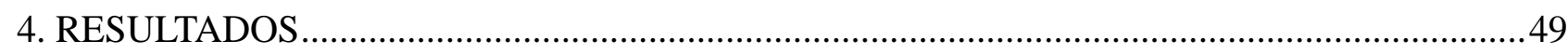

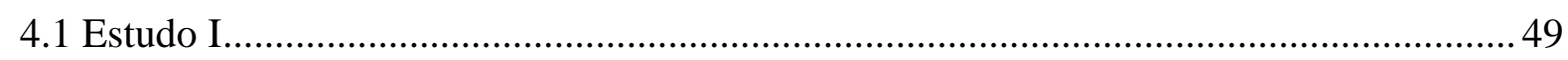

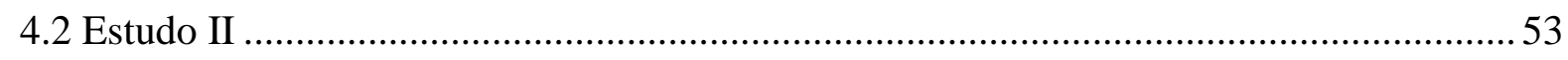

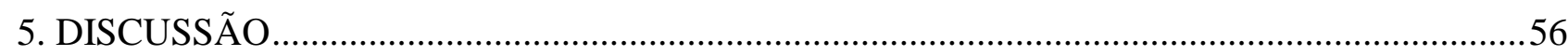

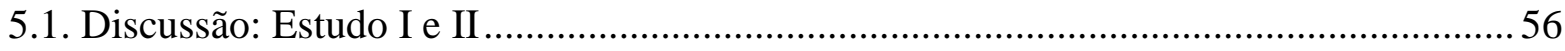

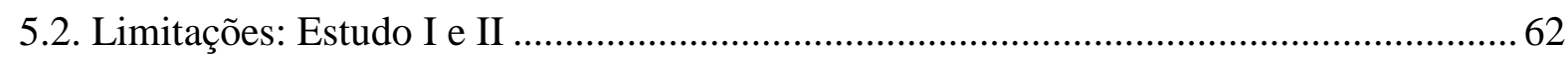

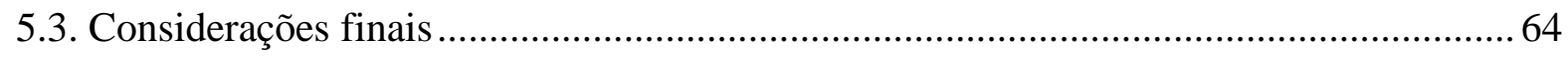

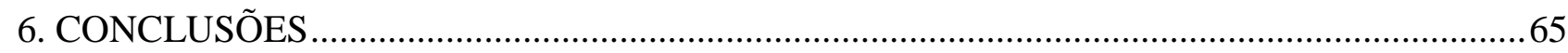

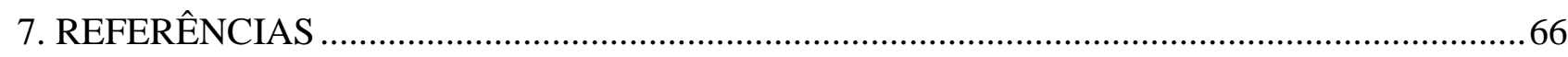

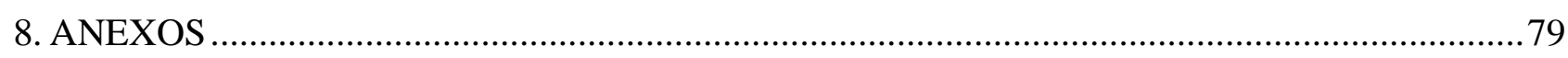




\section{LISTA DE SIGLAS E ABREVIATURAS}

ANVISA Agência Nacional de Vigilância Sanitária

CAGED Cadastro Geral de Empregados e Desempregados

CASA [Fundação] Centro de Atendimento Socioeducativo ao Adolescente

CBHPM Classificação Brasileira Hierarquizada de Procedimentos Médicos

CFP Conselho Federal de Psicologia

CID Classificação Internacional de Doenças

CMED Câmara de Regulação do Mercado de Medicamentos

CNPq Conselho Nacional de Desenvolvimento Científico e Tecnológico

DATASUS Dados do Sistema Único de Saúde

DAWBA Development and Well Being Assessment

DSM Manual Diagnóstico e Estatístico de Transtornos Mentais

FAPESP Fundação de Amparo a Pesquisa do Estado de São Paulo

FASE Fundação de Atendimento Socioeducativo

FHS Family History Screen

FLI Family Liability Index

FNDE Fundo Nacional de Desenvolvimento da Educação

FUNDEB Fundo de Manutenção e Desenvolvimento da Educação Básica e de Valorização dos Profissionais da Educação

HRC High Risk Cohort Stuy

ICMS Imposto Sobre Circulação de Mercadorias e Prestação de Serviços

INPD Instituto nacional de psiquiatria do desenvolvimento

IPCA Índice nacional de preços ao consumidor

PMC Preço máximo ao consumidor 
SOE Sem outra especificação

TDAH Transtorno de déficit de atenção e hiperatividade

TOC Transtorno obsessivo-compulsivo

YLD Years of life lost to disability 


\section{LISTA DE TABELAS}

Tabela 1: Unidades de custo do setor da saúde (em reais, valores de 2010) 46

Tabela 2: Unidades de custo do setor de serviços sociais e escolar (em reais, valores de 2010) .47

Tabela 3: Média de custos ao longo da vida (US\$ PPP) por criança por categoria de transtorno mental $(\mathrm{N}=2.512)$ .51

Tabela 4: Custo nacional ao longo da vida dos transtornos mentais subclínicos e clínicos na infância. .52

Tabela 5: Média de custos ao longo da vida (US\$ PPP) por criança e categoria de TOC $(\mathrm{N}=2512)$ .54

Tabela 6: Custo nacional ao longo da vida dos TOC subclínico e clínico. .55 


\section{LISTA DE FIGURAS}

Figura 1: Fluxograma da seleção amostral e etapas do HRC

Figura 2: Distribuição da estimativa do custo nacional dos transtornos mentais subclínicos e clínicos na infância. .52

Figura 3: Distribuição da estimativa do custo nacional do TOC subclínico e clínico na infância. .55 


\section{RESUMO}

Sá, DGF. Custo econômico e social do transtorno obsessivo-compulsivo e outros transtornos mentais na infância [tese]. São Paulo: Faculdade de Medicina, Universidade de São Paulo: 2016.

Os transtornos mentais na infância são prevalentes e causam prejuízo para o indivíduo, família e sociedade. Informações acerca do custo dos transtornos mentais na infância são úteis para o planejamento do sistema de saúde, para auxiliar tomadas de decisão de gestores acerca de investimentos na área e para determinação de prioridades no orçamento público. No entanto, não há dados sobre o custo dos transtornos mentais na infância no Brasil. Já dados sobre o custo dos transtornos mentais na infância em nível subclínico são inexistentes na literatura nacional e internacional. Os objetivos centrais da presente tese de doutorado, dividida em Estudo I e Estudo II, foram: estimar a média do custo do transtorno mental na infância em níveis subclínico e clínico, e estimar o custo total destes para o Brasil. O Estudo I teve como desfecho clínico qualquer diagnóstico de transtorno mental na infância; o Estudo II, o transtorno obsessivocompulsivo na infância (TOC), ambos em nível subclínico e clínico. A presente pesquisa é uma avaliação econômica de custo de doença de transtornos mentais na infância com utilização de método bottom-up retrospectivo. Foi baseada em dados de prevalência de um estudo populacional transversal de 2.512 estudantes de escolas públicas de Porto Alegre e São Paulo, selecionados por meio de duas técnicas: a) seleção aleatória de crianças e b) seleção de crianças com alto risco para desenvolvimento de transtornos mentais (baseado no histórico psiquiátrico familiar). Para avaliação de transtornos mentais na infância, foi utilizado o instrumento 
Development and Well Being Assesment (DAWBA). Os custos de doença foram estimados a partir dos seguintes componentes: tratamentos em saúde mental (uso de medicamentos, psicoterapia, hospitalização), uso de serviços sociais (assistência social, conselho tutelar, medidas socioeducativas) e problemas escolares (suspensão, abandono e repetência escolar). A amostra final foi de 2.512 crianças, de 6-14 anos de idade. Resultados do Estudo I: o transtorno mental infantil em nível subclínico e clínico teve média de custo total ao longo da vida de $\$ 1.750,86$ e $\$ 3.141,21$, respectivamente (todos os valores em PPP, purchasing power parity). O custo nacional estimado do transtorno mental subclínico foi de $\$ 9,92$ bilhões, enquanto do transtorno mental clínico foi de \$11,65 bilhões (baseado nos dados de prevalência do presente estudo). Resultados do Estudo II: o TOC subclínico e clínico apresentaram médias de custo total ao longo da vida de $\$ 1.651,81$ e $\$ 3.293,38$, respectivamente. O custo nacional do TOC subclínico foi de $\$ 6,71$ bilhões, enquanto do TOC clínico foi de $\$ 2,02$ bilhões (baseado nos dados de prevalência do presente estudo). Os dados apresentados nesta tese de doutorado fornecem evidências de que transtornos mentais subclínicos e clínicos na infância têm grande impacto econômico na sociedade. O conhecimento acerca do grande impacto econômico dos transtornos mentais na infância pode informar gestores e políticos sobre a magnitude do problema, de forma que seja possível planejar um sistema efetivo de cuidados com programas de tratamento e prevenção. Recomenda-se que gestores públicos aumentem os recursos para os setores da saúde e educação no Brasil, para promover prevenção e assistência em saúde mental da infância.

Descritores: 1. Custos e Análise de Custo, 2. Transtornos Mentais Diagnosticados na Infância, 3. Transtorno obsessivo-compulsivo, 4. Epidemiologia, 5. Estudos Transversais 


\section{ABSTRACT}

Fatori, D. Custo Econômico e Social do Transtorno Obsessivo-Compulsivo e Outros Transtornos Mentais na Infância [thesis]. São Paulo: Faculdade de Medicina, Universidade de São Paulo: 2016.

Child mental disorders are prevalent and impairing, negatively impacting families and society. Information on child mental disorders costs is important to plan the health system and to show policy makers how plan and prioritize budgets. However, there are no child mental disorders cost studies in Brazil. The main objectives of the present thesis were to estimate the mean costs of subthreshold and clinical mental disorders in children living in Brazil and to estimate its national costs. Outcome of Study I was any child mental disorder, outcome of Study II was child obsessive-compulsive disorder (OCD), both subthreshold and clinical. The present study it is cost-of-illness study of child mental disorders using a retrospective bottom-up methods, based on prevalence data from a cross-sectional study of children registered at public schools in Porto Alegre and Sao Paulo. A total of 8,012 families were interviewed, providing information about 9,937 children. From this pool, two subgroups were further investigated using randomselection $(n=958)$ and high-risk group selection procedure $(n=1,514)$, resulting in a total sample of 2,512 subjects 6-14 years old. Mental disorder assessment was made using the Development and Well-Being Assessment (DAWBA). The cost of child mental disorders was estimated from the following components: use of mental health services, social services and school problems. Costs were estimated for each child and the economic impact at the national level was calculated. Study I results: subthreshold and clinical disorder showed lifetime mean total cost of $\$ 1,750.86$ and $\$ 3,141.21$, 
respectively. The national lifetime cost estimate of clinical mental disorders in Brazil was $\$ 11.65$ billion, whereas for subthreshold mental disorder it was $\$ 19.92$ billion (all values in PPP, purchasing power parity). Study II: subthreshold and clinical OCD showed lifetime mean total cost of $\$ 1,651.81$ and $\$ 3,293.38$, respectively. The national lifetime cost estimate of clinical OCD in Brazil was $\$ 2.03$ billion, whereas for subthreshold OCD it was $\$ 6.71$ billion. The present study provides evidence about the economic impact of child mental disorders. This knowledge can inform about the magnitude of the problem, so that policy makers can make adjustments to better address these problems with cost-effective programs of treatment and prevention. It is recommended that health and education budgets in Brazil should increase to enhance prevention and treatment of children with childhood mental disorders.

Dscriptors: 1. Costs and cost analysis, 2. Mental disorders diagnosed in childhood, 3. Obsessive compulsive-disorder, 4. Epidemiology, 5. Cross-sectional studies 


\section{INTRODUÇÃO}

\subsection{A saúde mental na infância}

A saúde mental é prioridade na agenda global nos últimos anos (1). O paradigma contemporâneo de saúde tem como peça fundamental e indissociável a saúde mental (2). Esta importância se dá em razão do alto grau de limitação funcional e sofrimento causados pelos transtornos mentais. Sabe-se que $22,7 \%$ dos anos vividos com incapacidade pela população mundial atualmente (years of life lost to disability; YLD) são decorrentes de transtornos mentais. Desta carga, 9,6\% diz respeito aos transtornos depressivos, enquanto 3,5\% aos transtornos de ansiedade. Em 20 anos, período de 19902010, esta carga aumentou 37\% (3). Atualmente, estima-se que 13,4\% da população mundial de crianças e adolescentes tenha algum transtorno mental (4). Sabe-se também que a trajetória crônica é uma característica marcante dos transtornos mentais nessa faixa etária (5).

Estudos populacionais demonstram que frequentemente os transtornos mentais iniciam na infância e adolescência (6). Algumas crianças e adolescentes apresentam um conjunto de sintomas psicopatológicos, mas não preenchem os critérios necessários para diagnosticar um transtorno mental segundo os manuais diagnósticos. Apesar disso, o transtorno mental subclínico na infância e adolescência está associado com prejuízo funcional $(7,8)$, repetição escolar (9) e maior risco de desenvolver transtorno mental clínico no futuro (10-13). Casos identificados como subclínico frequentemente não recebem nenhum tratamento especializado $(14,15)$. 
A prevalência dos transtornos mentais subclínicos na infância e adolescência varia de 0,8\% a 23,1\% (16-20). Tamanho grau de variabilidade entre estudos se dá em razão da heterogeneidade de critérios de classificação do desfecho em questão. Definições acerca do número de sintomas necessários e prejuízo funcional são os principais critérios que costumam divergir entre os estudos. Atualmente, não existe um consenso acerca de uma definição de transtorno mental subclínico. Esta falta de consenso dificulta a pesquisa científica e prejudica a identificação de potenciais casos na comunidade e em serviços de saúde.

As versões mais recentes do Manual Diagnóstico e Estatístico de Transtornos Mentais (DSM) $(21,22)$ não disponibilizam uma categoria específica de transtorno mental subclínico, mas oferecem a possibilidade de classificação "Sem outra especificação" (SOE) ou "Não especificada(o)" para casos em que o paciente não preenche os critérios definidos, mas ainda assim apresenta conjunto relevante de sintomas e algum grau de prejuízo funcional, dessa forma, abarcando os casos subclínicos. Utilizando-se deste desfecho, um estudo longitudinal americano avaliou sujeitos dos nove aos 21 anos de idade e demonstrou uma prevalência cumulativa (ao longo da vida) de $82,5 \%$ de transtorno mental segundo critérios específicos e SOE (23).

A natureza categórica e o uso de limiares específicos da classificação diagnóstica do DSM trouxeram grandes avanços científicos e clínicos (24), contudo, as crescentes evidências acerca dos transtornos mentais subclínicos revelam uma falha dos manuais diagnósticos em não fornecer ferramentais suficientes para identificar casos que estão justamente abaixo dos limiares especificados (25), dificultando a detecção destes casos na comunidade e serviços de saúde. O uso das categorias SOE não oferece uma solução 
adequada e universal para esta questão, tanto para pesquisa, quanto para a clínica, justamente por não configurarem entidades nosológicas homogêneas. Outras modalidades de classificação poderiam oferecer potenciais soluções para este problema, como o uso do diagnóstico dimensional (puro ou híbrido) (26) ou o uso de um método ideográfico para maior precisão diagnóstica $(27,28)$.

Para além da complexa questão sobre os métodos de classificação diagnóstica, ressaltase a importância da compreensão contemporânea dos transtornos mentais como um contínuo entre saúde e doença, entre desenvolvimento típico e atípico. Pesquisas têm constantemente demonstrado que os transtornos mentais se iniciam precocemente $(5,6,29)$, frequentemente com manifestações subclínicas e/ou não-específicas (30) e prejuízo funcional já nos estágios iniciais (16,17,31).

Além dos prejuízos individuais na qualidade de vida da criança (32), e de efeitos negativos no funcionamento familiar (33), os transtornos mentais na infância têm associação com problemas de aprendizado (34), repetência e evasão escolar (35-37). Estes problemas, por sua vez, podem acarretar baixa escolaridade, resultando em baixos salários na idade adulta. Assim, observa-se que transtornos mentais, assim como outras doenças, geram problemas que perpassam o âmbito individual e familiar, determinando altos custos para a sociedade, governo e economia do país como um todo.

Estima-se que os custos dos transtornos mentais para a sociedade possam chegar até 226 bilhões de dólares anualmente por país, variando de acordo com a categoria diagnóstica (38). Estes ainda causam grande perda de produtividade mundialmente (39) e são responsáveis por uma redução de 0,3\%-0,8\% nos ganhos financeiros dos países (40). 
O levantamento dos custos econômicos dos transtornos mentais é imprescindível para se se compreender a magnitude do problema e, dessa forma, delimitar ações estratégicas para minimizar os impactos. Nessa perspectiva, o campo da avaliação econômica em saúde dispõe de ferramentas indispensáveis para que profissionais e gestores possam guiar suas condutas, aplicando de maneira eficaz os exíguos fundos disponíveis (41).

A pesquisa sobre o impacto econômico dos transtornos mentais na infância e adolescência tem crescido muito nos últimos anos. Uma ampla variedade de estudos tem sido publicada, focando em diversos diagnósticos ${ }^{1}$, como autismo $(42,43)$, transtorno de déficit de atenção e hiperatividade $(44,45)$, transtorno de conduta $(46-48)$, transtorno bipolar $(49,50)$, transtornos depressivos $(51-53)$, transtornos de ansiedade (54) e transtorno alimentar (55).

Estes estudos foram conduzidos em países de alta renda, principalmente Europa e Estados Unidos, sendo o Reino Unido o país com maior tradição nesta área específica do conhecimento. Logo, é evidente a falta de estudos em países de baixa e média renda, como o Brasil. Considerando que $46,6 \%$ da população de crianças e adolescentes do mundo vivem nos países menos desenvolvidos (56) é de suma importância que pesquisas sejam conduzidas para conhecer as realidades destes países. Além disso, é marcante a ausência de dados sobre outros transtornos mentais de alta relevância, em razão de gravidade e cronicidade, como o transtorno obsessivo-compulsivo (TOC).

\footnotetext{
${ }^{1}$ Busca realizada no PubMed com os seguintes termos: "Costs and Cost Analysis"[Mesh] AND "Mental Disorders"[Mesh] AND "infant"[MeSH Terms] OR "child"[MeSH Terms] OR "adolescent"[MeSH Terms].
} 
Com a finalidade de iniciar uma linha de pesquisa em avaliação de custos dos transtornos mentais no contexto do Instituto Nacional de Psiquiatria do Desenvolvimento (INPD, http://inpd.org.br/) e do Instituto de Psiquiatria (IPq) da Faculdade de Medicina da Universidade de São Paulo (FMUSP), decidiu-se analisar dois desfechos: ter qualquer transtorno mental e o TOC (ambos em nível clínico e subclínico). A presente tese teve como finalidade mais ampla, isto é, para além dos objetivos aqui propostos, desenvolver um framework para avaliação do custo dos transtornos mentais na infância. O estudo específico do TOC torna-se relevante justamente pela ausência de dados mesmo em países de alta renda, assim como por algumas de suas características: alta prevalência, início precoce, padrão de cronicidade e pela facilidade em se definir um contínuo psicopatológico entre os sintomas subclínicos e clínicos. Há uma literatura já consagrada tratando dessa temática especificamente em TOC, o que facilita o estabelecimento da definição do diagnóstico subclínico e comparação de dados.

\subsection{O transtorno obsessivo-compulsivo na infância}

O transtorno obsessivo-compulsivo é um transtorno mental caracterizado por comportamentos compulsivos e/ou pensamentos obsessivos (22). O prejuízo funcional e sofrimento causados pelo TOC são frequentemente graves e incapacitantes (57). A prevalência estimada do TOC na população de adultos é de 1,6\% (58), enquanto na população de crianças e adolescentes é de 2,7\% (59), taxas estas consideradas altas quando se têm em perspectiva os graves efeitos deletérios deste transtorno mental. 
O TOC na infância e adolescência tem características particulares se comparado à população adulta. A idade média de surgimento do quadro clínico é de 7,5-12,5 anos de idade (60), com predominância do sexo masculino quando o início é precoce (61). A qualidade de vida de crianças e adolescentes com este transtorno é ruim se comparada a controles saudáveis (62). Mais da metade dos pacientes com TOC na infância tem persistência dos sintomas na idade adulta, sendo que fatores como resposta inicial pobre ao tratamento, início precoce dos sintomas (63), severidade dos sintomas, comorbidades e sintomas proeminentes de colecionismo são características associadas à cronicidade $(64,65)$. Sabe-se ainda que pacientes com sintomas de inicio precoce apresentam maior chance de ter comorbidades com outros transtornos mentais $(66,67)$. Um estudo americano demonstrou que 97,5\% das crianças e adolescentes com TOC em uma amostra clínica tinham pelo menos uma comorbidade (61). O perfil cognitivo de pacientes com TOC na infância é um importante preditor do prognóstico e resposta ao tratamento, como déficits nos domínios: viso-espacial, habilidade motora fina e funções executivas $(68,69)$.

O TOC na infância e adolescência oferece um desafio clínico em relação ao processo de avaliação diagnóstica. A continuidade entre o desenvolvimento típico e o surgimento de sintomas obsessivo-compulsivos (SOC) configuram uma linha tênue. Por exemplo, hábitos restritos ou rituais, pode ser apenas parte do desenvolvimento típico. $\mathrm{Na}$ avaliação clínica um marcador importante para se considerar é o prejuízo funcional em termos de tempo gasto com os SOC e/ou sofrimento causado (70). Outro fator relevante na avaliação clínica tem relação com a natureza usualmente secreta dos sintomas. Pensamentos obsessivos ou rituais mentais podem gerar sensação de vergonha, assim, crianças têm uma tendência de não expressar verbalmente estas manifestações. Um 
estudo epidemiológico conduzido nos Estados Unidos demonstrou que avaliação por meio de entrevista parental resultou na identificação de apenas $0,3 \%$ dos casos de TOC na amostra e, adicionando informações da entrevista com criança/adolescente, a identificação de casos subiu para 2,7\% (59).

O tratamento do TOC na infância é embasado por ensaios clínicos randomizados apontando a eficácia da clomipramina e inibidores seletivos da recaptação de serotonina (ISRS; sertralina, fluoxetina, paroxetina e fluvoxamina) e terapia cognitivocomportamental (TCC) (71-73). A combinação de farmacoterapia e TCC também tem eficácia comprovada (73). Mais especificamente, no que tange a psicoterapia, a TCC é primeira linha no tratamento de TOC, demonstrando resultados eficazes há cinco décadas (74). Além da TCC individual, grupos de pesquisa têm demonstrado bons resultados à curto e longo prazo com TCC em grupo para o tratamento de TOC na infância $(75,76)$. Outros estudos também encontraram resultados positivos na aplicação de TCC em grupo no tratamento do TOC na infância (77-79).

Sabe-se que algumas crianças apresentam sintomas obsessivo-compulsivos, mas não preenchem critérios diagnósticos para TOC. Este fenômeno é comumente denominado TOC subclínico (em inglês, subthreshold ou subclinical). O conceito de TOC subclínico está alinhado à teoria da psicopatologia dimensional, na qual se entende que há um contínuo entre saúde-doença (26). Estudos mostram que crianças com TOC subclínico sofrem prejuízo funcional e possuem maior risco de desenvolver TOC clínico e outros transtornos mentais (11). Apesar de evidências demonstrarem que casos com TOC subclínico exibem prejuízo ou sofrimento, poucos obtém algum atendimento especializado. Um estudo brasileiro demonstrou que apenas $7 \%$ dos adolescentes com 
TOC subclínico procurou tratamento (15). Um outro estudo, na Espanha, revelou que $60 \%$ das crianças com TOC subclínico tiveram algum contato com serviço de saúde mental (14). A prevalência do TOC subclínico em crianças e adolescentes é maior que a do TOC clínico, e é estimada entre 5,5\% (14) e 18,3\% (15). Contudo, vale ressaltar que, como não há um consenso acerca da definição de TOC subclínico, os dados de prevalência variam amplamente.

A despeito dos graves efeitos deletérios do TOC no desenvolvimento de crianças, conforme mencionado previamente, não há dado demonstrando a magnitude do impacto econômico do TOC na infância. Para tal, faz-se necessário um tipo de estudo denominado estudo de custo de doença (cost-of-illness).

\subsection{O custo dos transtornos mentais: avaliação econômica em saúde mental}

O estudo de custo de doença visa identificar e medir a carga econômica imposta por uma determinada doença na sociedade. Tal informação tem o potencial de estimar o quanto uma doença custa para cada setor da sociedade, apontando possíveis prioridades para maiores investimentos ou controle de gastos $(80,81)$ e, também, estimar o quanto poderia ser economizado caso a doença em questão fosse erradicada ou minimizada. Em suma, dados acerca do custo de transtornos mentais são úteis para o planejamento do sistema de saúde e redes adjacentes.

Esta modalidade de pesquisa é uma das primeiras formas de avaliação econômica na área da saúde. Porém, até o final da década de 1960 a metodologia empregada nos estudos era heterogênea e pouco detalhada, resultando em dados pouco confiáveis e 
com possibilidade limitada de comparação. Os alicerces metodológicos mais rigorosos surgiram em 1967 com um estudo que identificou os custos das doenças descritas na Classificação Internacional de Doenças (CID) da época (82). Nos anos subsequentes, novos artigos foram publicados, impulsionando esta área ao aprimorar os métodos utilizados (83-85). O seminal estudo de Rice de 1967 apontou que os transtornos mentais representavam $15 \%$ do total de custos (diretos e indiretos) entre todas as categorias diagnósticas estudadas.

Os custos na área da saúde são comumente classificados em diretos e indiretos. Primeiramente, custos diretos se referem à utilização de recursos na área da saúde (ex. exames, medicamentos etc), internações hospitalares, utilização de recurso em outras áreas ligadas à saúde, transporte até o local de tratamento, entre outros (86). É possível ainda dividir estes por custos diretos médicos (ou da saúde) e diretos não-médicos.

Os custos indiretos se referem ao impacto na produtividade geral na vida do sujeito devido à morbidade (ex. incapacidade) e mortalidade (ex. perda de potencial produtivo) (87). Este conceito é derivado da teoria do capital humano e é frequentemente utilizado na literatura científica de avaliação econômica. Dentro desta ótica o indivíduo é considerado um fluxo de produção que tem seu valor na contribuição ao mercado, assim, o valor da vida é determinado pelo fluxo de ganhos futuros descontados. Nessa perspectiva a morbidade e a mortalidade prejudicam o trabalho (um recurso econômico importante) por causar perda de produção ou morte prematura. $\mathrm{O}$ custo indireto usualmente figura entre os maiores custos das doenças principalmente na área da saúde mental (Dorothy P. Rice, 1994; D. P. Rice, 1967). Por fim, alguns autores também descrevem um terceiro tipo de custos, denominado custo intangível. Este é definido 
como um aspecto da doença que não é passível de ser facilmente quantificado (dor psicológica, desconforto etc), logo, geralmente não é custeado (89).

No que tange o uso de dados epidemiológicos em estudos de custo de doença, existem duas possibilidades: prevalência e incidência. No método de prevalência, estima-se o custo da doença num período pré-determinado, usualmente de um ano. Assim, os custos pertencem apenas ao ano em que surgiram. Este método é interessante para demonstrar custos de uma doença que é subestimada pela sociedade e tomadores de decisão e para planejar políticas de contenção de custos $(80,90)$. No caso específico da saúde mental, que tem sua carga frequentemente subestimada pela sociedade e gestores, este método pode ser ideal.

Já o método de incidência visa identificar os custos a partir do surgimento da doença, dessa forma, analisa-se o fluxo de custos ao longo de um período de tempo determinado. Este método visa abarcar os diversos estágios e diferenças que podem ocorrer durante a trajetória temporal de uma doença. Este método é recomendado para planejar medidas preventivas e analisar manejo da doença do surgimento até cura ou morte (90). Tem como desvantagem a grande complexidade e custo de execução de um estudo longitudinal que possa fornecer os dados necessários.

A valoração dos custos ou custeio da doença estudada pode ser derivada de dois métodos: bottom-up e top-down. O método top-down (também chamado de grosscosting) foi preconizado por Dorothy Rice (82). Utiliza-se de dados secundários de gastos do sistema de saúde com as doenças de interesse (ex. consulta médica, internação). Assim, configura-se uma estimativa baseada na população, gerando médias 
de custos aproximadas. Os dados levantados são derivados de fontes secundárias e algumas limitações precisam ser consideradas, como possível alocação errônea de custos, exclusão de custos que não são do sistema de saúde (ex. transporte) e exclusão do custo de diagnósticos secundários (91).

O método bottom-up oferece um enfoque baseado no indivíduo, com dados coletados de fontes primárias por meio de instrumentos. É também chamado de micro-costing. O custo do uso de recursos em saúde e perda de produtividade é derivado de dados de pacientes com a doença de interesse. Define-se a priori as informações associadas ao desfecho clínico e, então, estima-se as unidades de custos. A valoração ocorre por meio da soma de componentes únicos de cada serviço utilizado, dessa forma, há uma aproximação maior do custo real. Este método tem como vantagem analisar de maneira detalhada diversos tipos de custos que usualmente não estariam disponíveis em fontes secundárias, sendo considerado o método preferencial em estudos de avaliação econômica (91). Ressalta-se que os dois métodos de valoração, bottom-up e top-down, podem ser usados num mesmo estudo de avaliação econômica, de acordo com a necessidade e disponibilidade de dados sobre uma determinada variável (90). Em estudos de custo de doença, o levantamento de dados via bottom-up é considerado o método mais acurado e confiável atualmente (89). Contudo, no contexto mais amplo da avaliação econômica em saúde, os métodos bottom-up e top-down têm sido discutidos e comparados e a escolha do método deve ser baseada na pergunta de pesquisa e necessidades e recursos do pesquisador $(92,93)$, assim, ambos os métodos possuem prós e contras que precisam ser levados em conta no planejamento de estudos. 
A coleta dos dados de custo pode ser realizada de maneira retrospectiva ou prospectiva. No caso da coleta retrospectiva os custos aconteceram antes do início do estudo. Possui vantagem por ser mais barato e rápido de se conduzir um estudo sob esta perspectiva. É indicado para doenças que possuem longa duração. No caso de coleta de dados primários há a limitação relativa ao viés de memória. É importante também o uso de instrumentos padronizados para minimizar viés de alocação de custos, no qual um determinado custo é erroneamente designado como decorrente do desfecho clínico de interesse $(89,90)$.

O método prospectivo levanta custos que ainda não aconteceram no momento de início do estudo. Nesta perspectiva é possível levantar dados mais acurados e detalhados acerca dos custos da doença. É possível o uso de diários para pacientes, o que aumenta confiabilidade dos dados. É indicado também para uma melhor compreensão de custos relativos a perda de produtividade, já que há maior possibilidade de levantar dados como ausência, atraso e licença médica, entre outros. A coleta de dados prospectiva não é usualmente indicada para doenças com longa duração devido ao aumento da complexidade e dificuldade de seguir os pacientes no tempo (89).

Estudos de custo de doença podem abordar os custos totais ou incrementais. A abordagem do custo total da doença se refere a uma estimativa do total dispendido em assistência à saúde das pessoas com a doença. Esta abordagem específica usa o método de soma de todos os custos médicos, que: identifica pessoas com o diagnóstico e adiciona todos os custos de cuidados em saúde independente do fato de serem ou não relacionados ao diagnóstico em questão. É a forma mais simples e é indicada para os desfechos clínicos sem ou com poucos custos não relacionados ao diagnóstico. É, ainda, 
possível usar o método de soma de diagnóstico específico, no qual se identificam pessoas com o diagnóstico primário em questão e somente custos diretamente relacionados à doença são computados. Há o risco de se subestimar custos caso ocorra uma falha em incluir indivíduos e custos "spillover" (custos indiretamente relacionados, mas que possuem relação com a doença primária) (90).

A abordagem dos custos incrementais se utiliza de uma estimativa do aumento de custos atribuído exclusivamente à presença do diagnóstico. Para tal, compara-se um grupo com a doença estudada, com um grupo controle pareado por características clínicas e/ou sociodemográficas. Por meio do método por controle pareado se calcula a média de todos os custos médicos entre as pessoas com o diagnóstico e subtrai a média dos mesmos tipos de custos entre os controles. Já o método por regressão se utiliza de um modelo estatístico de regressão para estimar os custos, podendo usar um grupo controle ou não (90).

No que tange o escopo dos custos a serem analisados, avaliações econômicas em saúde mental de crianças se diferenciam das realizadas na população adulta em razão da multiplicidade de contatos, serviços e tipos de tratamentos, característicos do atendimento a esta população $(94,95)$. Os principais fatores analisados em estudos que focaram crianças e adolescentes são: utilização de educação especial, reforço escolar, frequência escolar, aproveitamento escolar, repetência, expulsão, evasão escolar, benefícios governamentais recebidos pela família, uso de serviço social, estadia em abrigo, contato com o conselho tutelar, juizado ou promotoria, envolvimento com álcool, tabaco e outras drogas, acidentes, comportamento suicida, dias de trabalho perdidos pelos pais, desemprego dos pais, habitação (43-46,81,96-98). 


\subsection{Justificativa}

Nos países desenvolvidos há uma ampla literatura sobre o custo dos transtornos mentais, tanto na infância (44), como na idade adulta (99). No entanto, não há dados semelhantes na área da saúde mental da infância no Brasil. Considerando que a taxa de prevalência estimada de transtornos mentais em crianças e adolescentes no país é de 12,7-13,1\% (100,101), torna-se necessário compreender o custo econômico de desfechos clínicos tão prevalentes, justamente para determinar sua magnitude e para que haja um melhor planejamento de políticas públicas, organização de serviços e planejamento de gastos financeiros dos diversos setores relacionados à saúde mental. Além disso, até o momento, não há dados sobre o custo dos transtornos mentais subclínicos na infância na literatura internacional. Assim, o presente trabalho objetiva realizar a primeira investigação científica rigorosa sobre este tema no país e a primeira avaliação de custo dos transtornos mentais subclínicos na infância no mundo. 


\section{OBJETIVOS}

\subsection{Objetivos do Estudo I}

a) Estimar a média do custo de ter qualquer diagnóstico de transtorno mental na infância em nível subclínico e clínico nas cidades de São Paulo e Porto Alegre;

b) Estimar o custo nacional dos transtornos mentais subclínicos e clínicos na infância.

\subsection{Objetivos do Estudo II}

a) Estimar a média do custo de ter transtorno obsessivo-compulsivo (TOC) na infância em nível subclínico e clínico nas cidades de São Paulo e Porto Alegre;

b) Estimar a custo nacional do TOC subclínico e clínico na infância. 


\section{MÉTODO}

\subsection{Descrição geral}

A presente pesquisa é uma avaliação econômica de custo de doença dos transtornos mentais na infância ao longo da vida com utilização de método bottom-up retrospectivo, baseado em dados de prevalência de um estudo populacional transversal. A perspectiva da avaliação econômica foi da sociedade.

Este estudo faz parte do projeto "Análise Econômica de Intervenções para Prevenção e Tratamento de Transtornos em Saúde Mental de Crianças e Adolescentes”, um dos projetos do "Instituto Nacional de Ciência e Tecnologia de Psiquiatria do Desenvolvimento" (INPD; Processo CNPq: 08/57896-8; http://inpd.org.br/), financiado pelo Conselho Nacional de Desenvolvimento Científico e Tecnológico (CNPq) e pela Fundação de Amparo a Pesquisa do Estado de São Paulo (FAPESP). O INPD é um conjunto de iniciativas e projetos de pesquisa que busca introduzir ferramentas e métodos para promover o desenvolvimento saudável de crianças e adolescente por meio de uma rede que inclui pesquisadores de diversas universidades do Brasil e do mundo. Como pressuposto teórico, o INPD tem a 'Psiquiatria do Desenvolvimento': uma área interdisciplinar que visa compreender o desenvolvimento típico e atípico, a origem das suas alterações e a sua expressão como transtorno mental, com intuito de estabelecer novas estratégias de prevenção e tratamento para estes problemas.

O estudo "Análise Econômica de Intervenções para Prevenção e Tratamento de Transtornos em Saúde Mental de Crianças e Adolescentes" do INPD tem como objetivo 
a realização de avaliações econômicas - desde estudos de custo de doença até estudos de custo-efetividade - com base nos demais projetos contidos no INPD. Mais especificamente, a presente tese de doutorado se baseia no estudo epidemiológico "High Risk Cohort Study" (HRC, coorte de alto risco para transtornos psiquiátricos).

O HRC é um estudo epidemiológico que tem como objetivo geral identificar e caracterizar fenótipos e endofenótipos numa amostra de crianças com alto risco para desenvolver transtornos mentais. Uma ampla gama de fatores foi investigada, como: perfil sociodemográfico da família, fatores ambientais, uso de serviços de saúde, perfil neuropsicológico, exame fonoaudiológico e exame de ressonância magnética funcional.

O HRC teve aprovação da Comissão de Ética em Pesquisa da Faculdade de Medicina da Universidade de São Paulo. As entrevistas foram realizadas após o consentimento por escrito das mães/responsáveis, mantendo-se o sigilo das informações.

A seguir será descrita a primeira parte do estudo (wave 1), que foi divida em seis etapas, cujos dados foram utilizados nesta pesquisa. A figura 1, abaixo, apresenta o fluxograma dos participantes do estudo.

Etapa 1, rastreamento de famílias no dia da matrícula escolar: um total de 57 escolas (35 em São Paulo, 22 em Porto Alegre) foi incluído nesta etapa. Foram escolhidas escolas públicas com mais de mil alunos e que fossem localizadas em locais próximos dos centros de pesquisas devido a razões logísticas, principalmente no que tange a coleta de dados de neuroimagem. Os critérios de elegibilidade nessa etapa da pesquisa foram: (1) matrícula por responsável que fosse parente biológico e que fosse cuidador primário 
(para dar informações suficientes sobre o comportamento da criança); (2) criança com idade de 6-12 anos no momento da matrícula. No dia da matrícula todos os pais com crianças na faixa etária de interesse foram convidados a participar do estudo e aqueles que aceitaram participar responderam uma versão modificada do Family History Screen (FHS) (102) em entrevista no local ou posteriormente via telefone (19\% de entrevistas por telefone em Porto Alegre; em SP, todas por telefone). Ao fim dessa etapa, 8.012 famílias foram entrevistadas, provendo informações sobre 9.937 crianças. A mãe biológica foi a respondedora em $87,6 \%$ das entrevistas.

Etapa 2, seleção da amostra aleatória e de alto risco: utilizando-se os resultados do FHS, foi criado um índice de carga familiar representando a porcentagem de membros da família que positivaram para cada um dos transtornos mentais associados, ajustado por grau de parentesco (para contribuição genética no caso de meio-irmão). Os transtornos mentais de interesse que compuseram o índice: déficit de atenção e hiperatividade, ansiedade, obsessivo-compulsivo, sintomas psicóticos e transtornos de aprendizagem. As variáveis foram codificadas com zero (ausência) e 1 (presença). Aquelas crianças que positivaram para sintomas de qualquer um dos cinco transtornos mentais de interesse foram ranqueadas de acordo com a porcentagem de familiares que apresentavam sintomas do mesmo transtorno mental. Este índice foi desenvolvido baseado em trabalho previamente publicado (103).

$$
\text { Family Liability Index }(F L I)^{2}=\frac{M B+P B+\sum I B\left(\sum M I * 0,5\right)}{2+n I B+(n M I * 0,5)}
$$

\footnotetext{
${ }^{2}$ MB: mãe biológica; PB: pai biológico; IB: irmão biológico; MI: meio-irmão.
} 
Dentre as 9.937 crianças elegíveis, 1.500 foram selecionadas aleatoriamente para uma nova avaliação (sem substituição). Dentre as crianças restantes, aquelas que tinham sintomas relacionados aos cinco transtornos mentais de interesse foram listadas de acordo com o número de familiares com sintomas da mesma categoria diagnóstica (alto FLI). Assim, crianças desta subamostra de alto risco foram avaliadas até preencher o número fixo e pré-estabelecido de 2.512 (com uso de substituição; baseado em limite orçamentário do projeto). Por fim, a amostra final de 2.512 foi composta de 958 crianças selecionadas aleatoriamente e 1.554 pela estratégia de alto risco. Apenas uma criança por família foi selecionada; em famílias com mais de uma criança elegível, foi feita escolha aleatória da criança índice.

Etapa 3. entrevista domiciliar com pais conduzidas por entrevistador leigo: esta etapa consistiu na avaliação de fatores de risco para transtornos mentais, avaliação diagnóstica de transtorno mental na criança e nos pais e questões acerca de histórico de tratamento e uso de serviços de saúde e sociais por parte da criança $(\mathrm{N}=2.512)$. As variáveis utilizadas na análise do presente estudo foram coletadas nessa etapa do HRC.

Etapas 4, 5 e 6, avaliação neuropsicológica, fonoaudiológica, genética e de neuroimagem: demais etapas do estudo não estão relacionadas com o presente trabalho. Mais informações sobre a metodologia do HRC e estudos derivados da amostra podem ser encontradas em artigos publicados em periódicos internacionais $(31,104-113)$. 


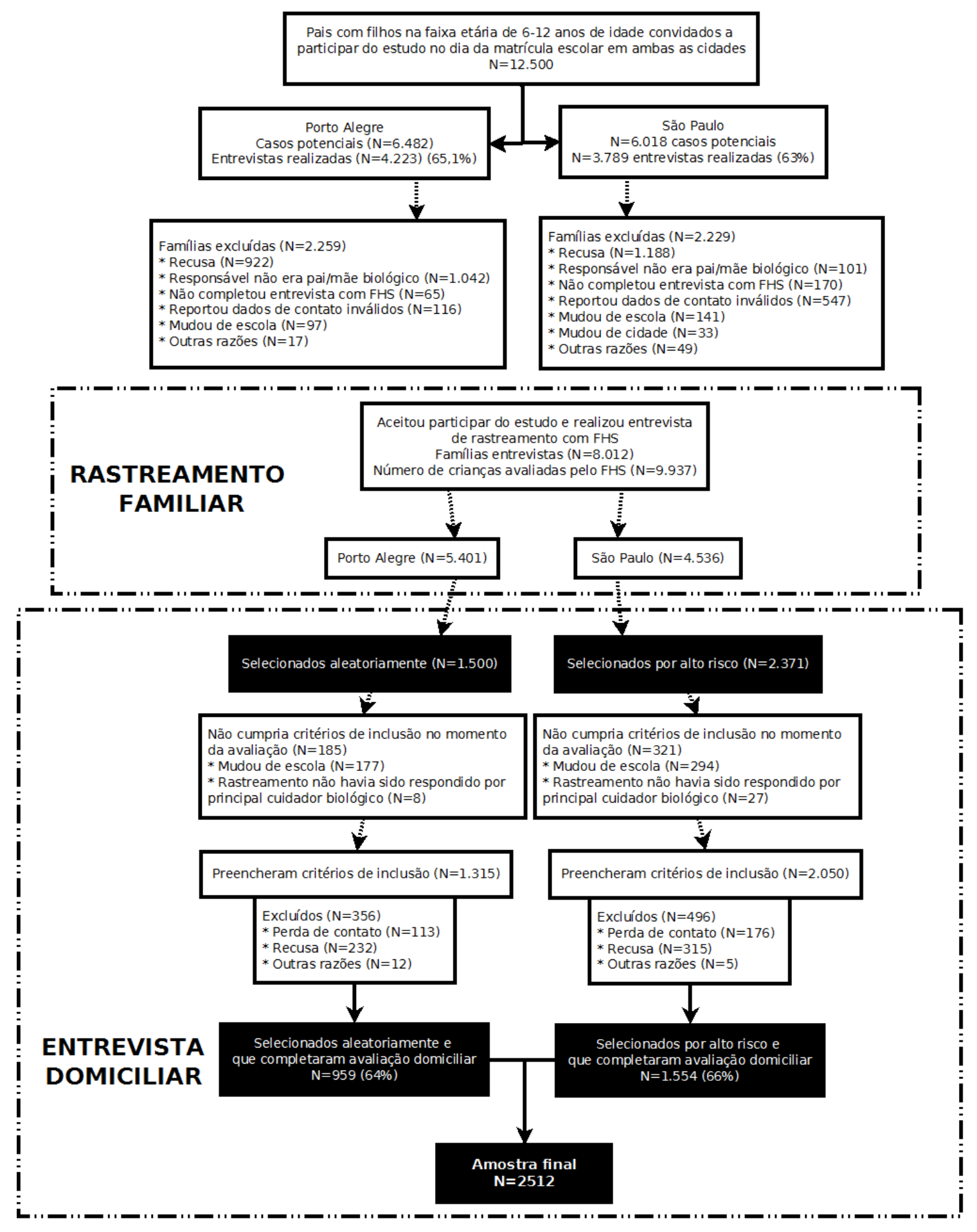

Figura 1. Fluxograma da seleção amostral e etapas do HRC. 


\subsection{Amostragem}

Como descrito acima, o processo de amostragem ocorreu por meio de uma triagem inicial, na qual 9.937 responsáveis por crianças de 6 a 12 anos foram entrevistados para levantamento de dados iniciais, no dia da matrícula de escolas públicas nas cidades de Porto Alegre e São Paulo. As escolas selecionadas situavam-se nas regiões do Hospital de Clínicas de Porto Alegre (da Universidade Federal do Rio Grande do Sul), do Hospital São Paulo (da Universidade Federal de São Paulo) e do Hospital das Clínicas da Faculdade de Medicina da Universidade de São Paulo. Nessas escolas foi feito um levantamento e 9.937 crianças na faixa etária desejada foram identificadas. Assim, os responsáveis foram entrevistados com intuito de se realizar uma triagem inicial acerca de sintomas psiquiátricos e históricos familiar de transtorno mental. Logo, a partir destes dados da triagem inicial, dois procedimentos de amostragem foram realizados: (1) seleção aleatória de crianças matriculadas nas escolas das cidades; (2) seleção de crianças com alto risco para desenvolvimento de transtornos mentais (histórico psiquiátrico familiar). Foram criadas listas de substituição para todas as crianças do segundo grupo, assim, em caso de recusa ou impossibilidade de localização, ocorria substituição do caso. No total, 1.343 substituições ocorreram para se chegar ao $\mathrm{N}$ final almejado. Por fim, a amostral final totalizou 2.512 crianças (958 por seleção aleatória e 1.554 em razão do alto risco para desenvolvimento de transtornos mentais).

\subsection{Entrevistadores}

Para a entrevista parental, um treinamento dos entrevistadores foi conduzido por psiquiatras clínicos experientes da equipe do estudo com duração de dois dias. O 
treinamento cobriu diversos tópicos: explicação acerca do estudo, principais aspectos dos transtornos mentais e fatores de risco, como lidar com situações difíceis ou complicadas durante a entrevista, questões éticas em pesquisa. Simulações e dramatizações de situações de entrevista foram realizadas. Todos os entrevistadores foram avaliados pela equipe do estudo para verificação de aptidão para realizar as entrevistas. Entrevistadores que não demonstraram aptidão para executar entrevistas de qualidade foram excluídos da pesquisa.

\subsection{Instrumentos}

Avaliação de transtornos mentais na infância/adolescência: Development and Well Being Assesment (DAWBA; Levantamento Sobre o Desenvolvimento e Bem-Estar de Crianças e Adolescentes; www.dawba.com). O DAWBA é uma entrevista estruturada desenvolvida para diagnosticar transtornos mentais e impacto dos sintomas em crianças e adolescentes de 5-17 anos segundo os critérios do DSM-IV-TR e CID-10 (114). Este instrumento foi traduzido e validado no Brasil e apresenta propriedades psicométricas satisfatórias (100). Os seguintes transtornos mentais são averiguados pelo DAWBA: anorexia nervosa, bulimia nervosa, fobia específica, fobia social, transtorno de estresse pós-traumático, transtorno de ansiedade de separação, transtorno de ansiedade generalizada, transtorno de pânico, agorafobia, transtorno obsessivo-compulsivo, transtorno depressivo maior, transtorno bipolar, transtorno de conduta, transtorno de déficit de atenção e hiperatividade, transtorno de oposição-desafiante, transtorno de tique e tourette, transtorno global do desenvolvimento. 
$\underline{\text { Levantamento de perfil sociodemográfico, uso de serviços de saúde e outras variáveis }}$ relacionadas ao custo de transtorno mental na infância/adolescência ${ }^{3}$ : foi utilizado um amplo questionário desenvolvido especificamente para o estudo por pesquisadores experientes na área da epidemiologia dos transtornos mentais da infância. Todas as questões se referiam ao longo da vida criança (ex. "a criança já fez alguma terapia psicológica ou já passou com psicólogo?” ou “a criança já tomou medicações para esses problemas?”).

\subsection{Desfecho clínico do Estudo I}

Para classificação diagnóstica do Estudo I as DAWBA bands, uma medida categóricaordinal gerada via um algoritmo computadorizado que define a probabilidade de uma criança ter um diagnóstico positivo baseado nos dados de sintomas e/ou prejuízo funcional. Um estudo realizado pelos autores do instrumento demonstrou que as DAWBA bands possuem uma relação de dose-resposta com uso de serviços, fatores de risco e proteção (115). As seis categorias originais de probabilidade foram convertidas em três categorias para as análises do Estudo I: ausência de transtorno mental $\left(<0,1 \%{ }^{4}\right.$, 0,5\% e $3 \%$ ), presença de qualquer transtorno mental subclínico (15\%) e presença de qualquer transtorno mental clínico $(50 \%$ e $>70 \%)$.

\subsection{Desfecho clínico do Estudo II}

Todos os diagnósticos de transtorno mental seguiram rigorosamente os procedimentos padronizados do instrumento DAWBA, com exceção do TOC. Membros do comitê do

3 Anexos A e B contém a distribuição dos problemas escolares, uso de serviços sociais e uso de tratamentos de saúde por transtorno mental na infância.

${ }^{4}$ Nenhuma criança da amostra foi classificada nessa categoria. 
HRC ajustaram o algoritmo diagnóstico do DAWBA para melhor representar a realidade do quadro clínico deste transtorno mental na infância. Os seguintes itens foram inclusos: presença de qualquer obsessão ou compulsão aliada às medidas de consumo de tempo (mais de uma hora ao dia) (item F11), interferência (F12), sofrimento e aborrecimento causado pelos sintomas (F10; F13). Por fim, foram excluídos os itens: os "sintomas são excessivos ou irracionais" (por não se aplicar às crianças) (F8), "resistência aos sintomas" (F9), bem como a necessidade do preenchimento do intensificador de sintomas ("muito", "extremamente") do DAWBA nos itens F10, F12 e F13. Maiores detalhes acerca do processo relativo à definição de TOC e SOC podem ser lidos em outro trabalho (116). Ao final do processo, a taxa de prevalência de TOC encontrada foi de 3,07\% para amostra total e 2,1\% para amostra ponderada, taxa esta semelhante a dados encontrados na literatura nacional e internacional $(15,59)$.

O quadro subclínico de TOC foi definido a partir da presença de sintomas obsessivocompulsivos (SOC), sem a presença do diagnóstico de TOC. Assim, a presença de qualquer item obsessão e/ou compulsão pela seção $\mathrm{F}$ do DAWBA, que incluem a presença de rituais nas últimas quatro semanas (item F2) ou presença de obsessõespreocupações (itens F3, F4). Foi, então, criada uma variável com as seguintes categorias: (0) ausência de transtorno mental, (1) outros transtornos mentais, (2) presença de TOC subclínico e ausência de outros transtornos mentais, e (3) presença de TOC clínico e outros transtorno mentais. 


\subsection{Ponderação dos desfechos (Estudo I e II)}

No intuito de ajustar a amostra em razão da estratégia de amostragem de alto risco, as variáveis do FHS que geraram o FLI, assim como número de sintomas das crianças, foram utilizadas em uma análise de propensity score matching (117) em que as 2.371 crianças selecionados a participarem do estudo foram pareadas com as 1500 crianças selecionadas aleatoriamente. $\mathrm{O}$ percentual de melhora do balanceamento da amostra (aleatória vs. alto risco) ficou entre $61,8 \%$ e 96,8\%, exceto para a categoria de sintomas psicóticos. O propensity score, baseado na análise de pareamento, foi utilizado como peso (weight) para ponderação das prevalências obtidas por meio do DAWBA (desfecho estudo I e II). As prevalências de transtorno mental eram de 19,9\% (grupo aleatório) e 29,7\% (grupo alto risco) antes da ponderação. Posteriormente, com a ponderação, as prevalências de transtorno mental ficaram: 19,9\% (grupo aleatório) e 20\% (grupo alto risco).

\subsection{Estimação dos custos (Estudo I e II)}

A tabela 1 e 2 apresentam as unidades de custo dos setores da saúde, de serviços sociais e escolar. A seguir será descrita a metodologia de custeio de cada uma das variáveis dos estudos I e II: 
Tratamento para transtornos mentais, medicamentos psiquiátricos:

Uma lista de medicamentos específicos da área da saúde mental (anexo E) foi apresentada aos entrevistados e estes relatavam se a criança já tinha usado qualquer um destes e por quanto tempo (em meses).

O custeio dos medicamentos se baseou na lista de preços de medicamentos da "Câmara de Regulação do Mercado de Medicamentos" (CMED), disponibilizada todos os anos pela "Agência Nacional de Vigilância Sanitária" (ANVISA). Esta lista apresenta os preços tabelados de todos os medicamentos disponíveis e regulamentados no mercado brasileiro. O custeio foi realizado por meio do "preço máximo ao consumidor" (PMC) com a menor taxa do "Imposto Sobre Circulação de Mercadorias e Prestação de Serviços" (ICMS) disponível para cada medicamento para o ano de 2010. O PMC foi multiplicado pelo número de meses de uso do medicamento, considerando a menor dose terapêutica possível de cada medicamento.

O número de consultas médicas necessárias para prescrição do medicamento foi estimado em uma consulta a cada três meses. O valor da consulta médica ( $\mathrm{R} \$ 67,82)$ foi baseado na "Classificação Brasileira Hierarquizada de Procedimentos Médicos" (CBHPM), de 2010, que é válida para todo território nacional (tabela 1).

$\underline{\text { Tratamento para transtornos mentais, psicoterapia: }}$

Foi perguntado aos responsáveis se a criança já realizou psicoterapia em algum momento da vida, qual tipo de profissional realizou o tratamento (psicólogo, psiquiatra, 
terapeuta ocupacional, terapeuta de família e outro) e por quanto tempo o tratamento foi realizado (em meses).

O valor da sessão de psicoterapia realizada por psicólogos e terapeuta de família foi definido pelo valor mínimo $(\mathrm{R} \$ 81,62)$ recomendado pelo "Conselho Federal de Psicologia" (CFP), válido para todo o território nacional ${ }^{5}$. Para psicoterapia realizada por profissional médico, o valor da consulta $(\mathrm{R} \$ 67,82)$ foi baseado na tabela CBHPM, valor de 2010.

No caso de terapeuta ocupacional, foi levantado o salário médio por meio do "Cadastro Geral de Empregados e Desempregados" (CAGED) no ano de 2010, assim, foi calculado o valor da sessão/hora baseado na carga horária de 30 horas/semana para cada cidade (R\$56,43 para São Paulo e R \$72,19 Porto Alegre).

Tratamento para transtornos mentais, internação hospitalar:

Dados acerca do número de vezes que a criança foi internada por conta de transtorno mental em hospital ou clínica foram coletados. Levantou-se, por meio do DATASUS (Dados do Sistema Único de Saúde), o custo médio da internação hospitalar na faixa etária de 5-14 $\operatorname{anos}^{6}$ em razão de diagnóstico do capítulo V (transtornos mentais e comportamentais, F00-F99) da Classificação Internacional de Doenças (CID-10) na cidade de São Paulo e Porto Alegre (tabela 1).

\footnotetext{
${ }^{5} \mathrm{O}$ CFP não fornece o dado específico de cada ano. Ao ser questionado via e-mail acerca dos dados de 2010, apenas enviaram novamente os dados atuais que estão no website do CFP.

${ }^{6}$ Faixa etária disponível mais próxima da presente amostra.
} 
Uso de serviços sociais, assistência social e conselho tutelar:

O número de contatos com o conselho tutelar e assistência social foi baseado em um estudo populacional prévio conduzido na atenção primária do município de São Paulo, já que o protocolo de pesquisa não contemplou o número de vezes e duração do atendimento nestes serviços. Na amostra do estudo mencionado crianças (6-11 anos) tiveram uma média de três contatos com o conselho tutelar e cinco com a assistência social (estudo referente ao anexo D). As unidades de custo foram estimadas a partir dos valores dos salários médios do assistente social e do conselheiro tutelar, levantados via CAGED (ano de 2010) para ambas as cidades da pesquisa. O valor da sessão/hora foi baseado na carga horária de 40 horas/semana para cada cidade.

Uso de serviços sociais, medidas socioeducativas:

O custo do interno (média mensal) no ano de 2010 na "Fundação Centro de Atendimento Socioeducativo ao Adolescente" (CASA) foi obtido via portal de transparência do Governo do Estado de São Paulo. O custo do interno na "Fundação de Atendimento Socioeducativo" (FASE), órgão do Rio Grande do Sul, não foi encontrado disponível na internet e, por e-mail, responsáveis da instituição se negaram a revelar o valor. Assim, o custo da Fundação CASA $(\mathrm{R} \$ 6.478,06)$ foi usado como estimativa para custo do interno na FASE. A estimativa de tempo de internação para aplicação de medidas socioeducativas, segundo dado repassado pela Fundação CASA, é de 10-12 meses, então, utilizou-se o tempo de 10 meses para fins de custeio da variável. 
Problemas escolares, suspensão, repetência e abandono escolar:

Os problemas escolares foram escolhidos para representar a dimensão de custos indiretos dos transtornos mentais da infância. Como base do custeio das variáveis escolares foram utilizados dados do ano de 2010 do "Fundo de Manutenção e Desenvolvimento da Educação Básica e de Valorização dos Profissionais da Educação" (FUNDEB) do "Fundo Nacional de Desenvolvimento da Educação" (FNDE). O FNDE divulga anualmente dados do custo por aluno no sistema de ensino público para todos os Estados brasileiros (tabela 2). No caso de repetência e abandono escolar (definida como mais de um mês sem ir à aula, sem retornar), utilizou-se o valor anual por aluno multiplicado pelo o número de vezes que a criança repetiu de série ou tempo de abandono escolar. No caso da suspensão escolar, estimou-se um tempo de suspensão de cinco dias, calculado a partir do valor do dia letivo (valor anual dividido por 200 dias letivos, R \$11,59 para São Paulo e R \$10,02 para Porto Alegre).

Perda de produtividades dos pais:

O custo da perda de produtividade dos pais foi estimado como custo indireto baseado na teoria do capital humano (118). Assim, para cada consulta médica, psicológica e atendimento em serviços sociais (assistência social, conselho tutelar) da criança foi descontado o equivalente a duas horas da remuneração do cuidador principal. A remuneração do cuidador foi baseada no relato do entrevistado e foram considerados apenas aqueles que estavam empregados. 
Tabela 1. Unidades de custo do setor da saúde (em reais, valores de 2010).

\begin{tabular}{ccc}
\hline Profissionais e serviços da saúde & São Paulo, SP & Porto Alegre, $\mathbf{R S}$ \\
\hline Consulta médica (em consultório) $^{\mathrm{a}}$ & 67,82 & 67,82 \\
Internação psiquiátrica no SUS (média) $^{\mathrm{b}}$ & 526,13 & $1.137,47$ \\
Psicoterapia individual $^{\mathrm{c}}$ & 81,62 & 81,62 \\
Terapeuta ocupacional $^{\mathrm{d}}$ & 56,43 & 72,19 \\
\hline
\end{tabular}

Medicamentos $^{\mathrm{e}}$ (unidade/caixa)

Fluoxetina

12,28

Fluvoxamina

70,46

Paroxetina

13,58

Sertralina

14,69

Clomipramina

6,52

Imipramina

4,5

Amitriptilina

7,28

Bupropiona

59,28

Metilfenidato

17,92

Clonazepam

Diazepam

Zolpidem

16,4

Carbamazepina

3,84

Oxcarbamazepina

29,92

Valproato

6,14

Lamotrigina

17,87

Fenitoína

4,73

Clorpromazina

Levomepromazina

Haloperidol

Quetiapina

Risperidona

Lítio

${ }^{a}$ Classificação Brasileira Hierarquizada de Procedimentos Médicos (CBHPM), dados de 2010, válidos para todo território nacional (fonte: http://www.amb.org.br/Site/Home/PUBLICA\%C3\%87\%C3\%95ES\%20AMB/CBHPM/)

${ }^{\text {b }}$ Dados do Sistema Único de Saúde (DATASUS), ano de 2010, internação para faixa etária 5-14, todos os diagnósticos do CID cap. 5 (fonte: http://www2.datasus.gov.br/DATASUS/index.php)

${ }^{c}$ Conselho Federal de Psicologia (CFP), valor mínimo, válido para todo o território nacional. (fonte: http://site.cfp.org.br/servicos/tabela-de-honorarios/)

${ }^{d}$ Cadastro Geral de Empregados e Desempregados (CAGED), salário médio admissional, ano 2010 (fonte: http://portal.mte.gov.br/caged/cadastro-geral-de-empregados-e-desempregados-caged.htm)

e Câmara de Regulação do Mercado de Medicamentos (CMED), Agência Nacional de Vigilância Sanitária (ANVISA) 
Tabela 2. Unidades de custo do setor de serviços sociais e escolar (em reais, valores de 2010).

\begin{tabular}{ccc}
\hline Serviços sociais & São Paulo, SP & Porto Alegre, RS \\
\hline Assistente Social (valor por hora) ${ }^{\mathrm{a}}$ & 12,75 & 11,26 \\
Conselheiro Tutelar (valor por hora) $^{\mathrm{a}}$ & 3,72 & 3,59 \\
Internação na Fundação CASA por adolescente $^{\mathrm{b}}$ & \multicolumn{2}{c}{$6.478,06$} \\
(média mensal) $^{\mathrm{b}}$ & \multicolumn{2}{c}{} \\
\hline
\end{tabular}

\section{Escola}

\begin{tabular}{l}
$\begin{array}{l}\text { Custo anual por aluno do ensino fundamental } \\
\text { público }^{c}\end{array}$ \\
\hline
\end{tabular}

${ }^{\text {a }}$ Cadastro Geral de Empregados e Desempregados (CAGED), salário médio admissional, ano 2010 (fonte: http://portal.mte.gov.br/caged/cadastro-geral-de-empregados-e-desempregados-caged.htm)

${ }^{\mathrm{b}}$ Fundação Centro de Atendimento Socioeducativo ao Adolescente (CASA), dados de 2010, obtidos via e-mail.

${ }^{c}$ Fundo de Manutenção e Desenvolvimento da Educação Básica e de Valorização dos Profissionais da Educação (FUNDEB), dados para o ano de 2010 (fonte: http://www.fnde.gov.br/financiamento/fundeb/fundeb-apresentacao)

\subsection{Análise dos dados}

Os dados de custos foram corrigidos pela inflação (período 2010-2015) usando o índice nacional de preços ao consumidor (IPCA) (119). Posteriormente, os custos foram convertidos para paridade do poder de compra (purchasing power parity, PPP) usando o último ano em que o fator de conversão estava disponível (2014). O PPP é um método alternativo à taxa de câmbio com intuito de viabilizar comparações de dados entre países. A taxa de conversão PPP se refere ao número de unidades de uma determinada moeda (no presente trabalho, o Real) necessárias para comprar uma mesma quantidade de bens ou serviços em relação ao dólar $(120,121)$. As médias dos custos foram calculadas em Real, depois corrigidas e convertidas para PPP.

Considerando a distribuição não-normal usualmente observada em dados econômicos, assim como a incerteza estatística acerca de medidas de tendência central, intervalos de confiança de $95 \%$ e erros padrões foram gerados por meio do método bootstrapping 
com 1000 replicações de amostragem aleatória com substituição para todas as médias calculadas (122).

As estimativas dos custos nacionais, isto é, a extrapolação dos dados para todo o território brasileiro, foram baseadas no último censo populacional realizado pelo Instituto Brasileiro de Geografia e Estatística (123). Então, as médias dos custos foram multiplicadas pelo número estimado de crianças com transtorno mental. Para o Estudo I, presença de transtorno mental subclínico e clínico baseado nas DAWBA bands, e para o Estudo II, presença de TOC subclínico e clínico segundo avaliação clínica do DAWBA. Todas as prevalências utilizadas para obtenção do custo nacional foram ajustadas por ponderação (weights) conforme descrito previamente.

Todas as variáveis de custos dos transtornos mentais na infância foram computadas e calculadas por meio do programa "Statistical Package for the Social Sciences" versão 22 (SPSS 22). Foram calculadas médias, desvios-padrão (DP), intervalos de confiança e soma das variáveis de interesse. Por fim, ressalta-se que os custos se referem sempre ao longo da vida, devido ao questionário de uso de serviços e avaliação econômica se referir ao longo da vida da criança. 


\section{RESULTADOS}

\subsection{Estudo I}

A prevalência de transtorno mental subclínico e clínico foi de 19,4\% (N=409) e 12,7\% ( $\mathrm{N}=268)$, respectivamente (prevalências ponderadas). Cinquenta e três por cento da amostra era do sexo masculino. O anexo A demonstra a distribuição dos problemas escolares, uso de serviços sociais e uso de tratamentos de saúde por transtorno mental na infância para fins de verificação dos dados. A tabela 3 apresenta a média dos custos (US\$ PPP) ao longo da vida por transtorno mental infância. Transtorno mental infantil em nível subclínico e clínico teve custo médio ao longo da vida de \$1.750,86 $(\mathrm{DP}=5.844,9)$ e $\$ 3.141,21$ ( $\mathrm{DP}=6.890,9)$, respectivamente. A média do custo da saúde de ter qualquer transtorno mental clínico foi 9,2 vezes maior do que não ter transtorno mental. Já média do custo total de ter qualquer transtorno mental clínico foi 2,7 vezes maior do que não ter nenhum transtorno mental. Na categoria de custos relacionados à saúde, ter qualquer transtorno mental subclínico se mostrou 4,3 vezes maior do que não ter transtorno mental.

O custo nacional estimado, somando o custo da saúde, problemas escolares, serviço social e perda de produtividade dos pais, do transtorno mental subclínico foi de $\$ 9,92$ bilhões, enquanto do transtorno mental clínico na infância foi de $\$ 11,65$ bilhões (tabela 4). Ambas as categorias somaram $\$ 21,57$ bilhões. O setor de saúde abarcou $44 \%$ e $53 \%$ dos custos nacionais do transtorno mental subclínico e clínico, respectivamente. Os problemas escolares configuraram o segundo maior custo entre crianças com transtorno mental, nível subclínico e clínico, enquanto perda de produtividade dos pais e uso de 
serviços sociais representaram apenas $5,2 \%$ dos custos de ter transtorno mental clínico cada

uma

(figura

$1)$. 
Tabela 3. Média de custos ao longo da vida (US\$ PPP) por criança por categoria de transtorno mental (N=2512).

\begin{tabular}{|c|c|c|c|c|c|c|c|c|c|}
\hline & \multicolumn{3}{|c|}{$\begin{array}{l}\text { Ausência de transtorno mental } \\
\qquad(\mathrm{N}=1569)\end{array}$} & \multicolumn{3}{|c|}{$\begin{array}{l}\text { Qualquer transtorno mental } \\
\text { subclínico }(\mathrm{N}=\mathbf{5 1 1})\end{array}$} & \multicolumn{3}{|c|}{$\begin{array}{l}\text { Qualquer transtorno mental } \\
\text { clínico }(N=432)\end{array}$} \\
\hline & \multirow[b]{2}{*}{ Média } & \multicolumn{2}{|c|}{$95 \%$ IC } & \multicolumn{3}{|c|}{$95 \%$ IC } & \multirow[b]{2}{*}{ Média } & \multicolumn{2}{|c|}{$95 \%$ IC } \\
\hline & & Inferior & Superior & Média & Inferior & Superior & & Inferior & Superior \\
\hline Saúde & 180,50 & 111,26 & 255,91 & 777,25 & 467,67 & 1172,49 & 1676,22 & 1243,30 & 2154,05 \\
\hline Medicamento & 5,08 & 2,57 & 8,02 & 44,08 & 15,25 & 86,20 & 119,85 & 71,76 & 176,12 \\
\hline Consulta médica & 7,80 & 3,76 & 13,87 & 28,32 & 13,23 & 49,37 & 107,39 & 66,95 & 152,90 \\
\hline Psicoterapia & 166,25 & 107,39 & 241,59 & 704,84 & 416,13 & 1075,18 & 1448,98 & 1071,58 & 1859,27 \\
\hline Serviços sociais & 469,84 & 235,46 & 741,26 & 131,13 & 9,89 & 381,88 & 162,63 & 17,83 & 474,45 \\
\hline Assistente social & 1,15 & 0,85 & 1,45 & 2,32 & 1,57 & 3,08 & 4,13 & 3,08 & 5,20 \\
\hline Conselho tutelar & 0,40 & 0,28 & 0,54 & 0,80 & 0,50 & 1,13 & 1,45 & 0,99 & 1,95 \\
\hline Medida socioeducativa & 463,63 & 230,06 & 718,07 & 118,63 & 110,70 & 469,01 & 140,33 & 130,92 & 546,89 \\
\hline Problemas escolares & 468,81 & 409,25 & 534,04 & 739,10 & 598,60 & 885,07 & 1154,32 & 946,07 & 1355,47 \\
\hline Repetência & 411,34 & 362,20 & 464,37 & 608,09 & 499,74 & 727,91 & 919,77 & 777,79 & 1068,45 \\
\hline Suspensão & 16,02 & 5,33 & 29,25 & 13,04 & 2,34 & 32,48 & 32,15 & 7,15 & 69,22 \\
\hline Evasão & 41,45 & 19,02 & 67,83 & 117,97 & 49,35 & 197,14 & 202,40 & 102,35 & 323,14 \\
\hline $\begin{array}{l}\text { Perda de produtividade } \\
\text { parental }\end{array}$ & 65,02 & 55,33 & 79,79 & 112,76 & 77,36 & 174,37 & 164,76 & 120,87 & 215,26 \\
\hline TOTAL & 1179,51 & 914,88 & 1467,57 & 1750,86 & 1301,89 & 2236,95 & 3141,21 & 2592,83 & 3821,21 \\
\hline
\end{tabular}

$\mathrm{PPP}=$ power purchase parity, $\mathrm{IC}=$ intervalo de confiança 
Tabela 4. Custo nacional ao longo da vida dos transtornos mentais subclínicos e clínicos na infância.

\begin{tabular}{|c|c|c|}
\hline \multirow[b]{2}{*}{ Tipo de custo } & \multicolumn{2}{|c|}{ Estimativa do custo nacional (US\$ PPP bilhão) } \\
\hline & $\begin{array}{c}\text { Transtorno mental } \\
\text { subclínico }\end{array}$ & $\begin{array}{c}\text { Transtorno mental } \\
\text { clínico }\end{array}$ \\
\hline Saúde & 4,4 & 6,22 \\
\hline Serviços sociais & 0,74 & 0,6 \\
\hline Problemas escolares & 4,19 & 4,28 \\
\hline Perda de produtividade parental & 0,64 & 0,61 \\
\hline TOTAL & 9,92 & 11,65 \\
\hline
\end{tabular}

$\mathrm{PPP}=$ power purchase parity

Figura 2. Distribuição da estimativa do custo nacional dos transtornos mentais subclínicos e clínicos na infância.

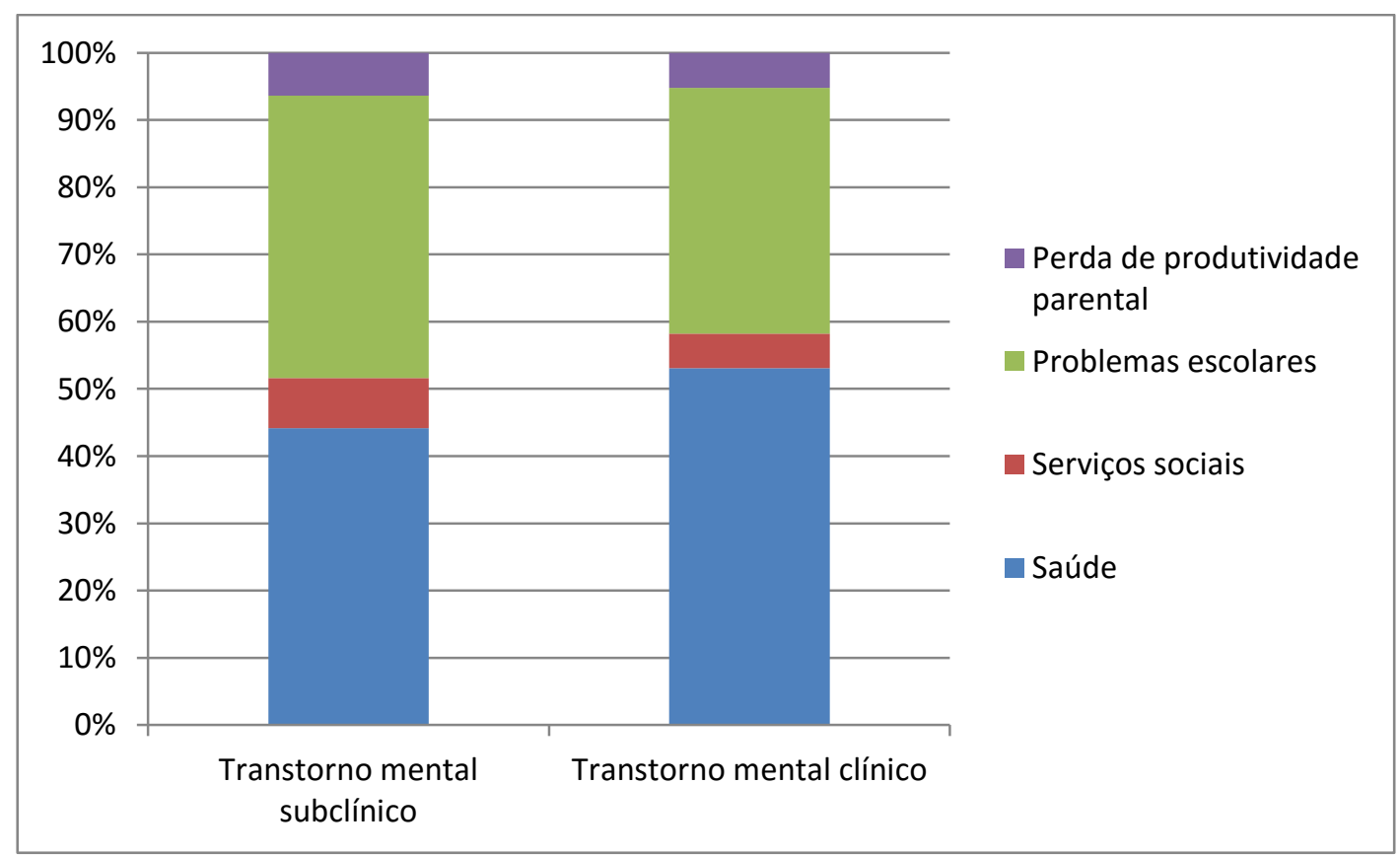




\subsection{Estudo II}

A prevalência de TOC subclínico na amostra foi de 13,9\% e 2,1\% para TOC clínico (prevalência ponderada; todos os casos de TOC clínico apresentaram comorbidade). O anexo B apresenta a distribuição dos problemas escolares, uso de serviços sociais e uso de tratamentos de saúde por transtorno mental na infância para fins de averiguação dos dados relativos às variáveis econômicas. O TOC subclínico e clínico apresentaram médias de custo total ao longo da vida de $\$ 1.651,81$ ( $\mathrm{DP}=7.583,6)$ e $\$ 3.293,38$ $(\mathrm{DP}=8.680,4)$, respectivamente. Diferenças encontradas entre as categorias diagnósticas foram substanciais. A média de custo da saúde do TOC clínico foi 7,2 vezes maior do que a média da ausência de transtorno mental. A média de custo do TOC subclínico se mostrou maior que ausência de transtorno mental nas categorias serviço social, problemas escolares e no custo total (tabela 5).

O custo nacional estimado para a população de 6-14 anos de idade do TOC subclínico foi de $\$ 6,71$ bilhões, enquanto do TOC clínico foi de $\$ 2,02$ bilhões (tabela 6). Ambas as categorias somaram $\$ 8,73$ bilhões. O setor da saúde abarcou $61 \%$ dos custos totais do TOC clínico. A categoria problemas escolares foi a segunda maior contribuidora do custo total do TOC subclínico. O uso de serviços sociais representaram $47 \%$ dos custos do TOC subclínico (figura 2). 
Tabela 5. Média de custos ao longo da vida (US\$ PPP) por criança e categoria de TOC (N=2512).

\begin{tabular}{|c|c|c|c|c|c|c|c|c|c|c|c|c|}
\hline & \multicolumn{3}{|c|}{$\begin{array}{l}\text { Ausência TM } \\
\qquad(\mathrm{N}=1518)\end{array}$} & \multicolumn{3}{|c|}{$\begin{array}{c}\text { Outros TM } \\
(\mathbf{N}=600)\end{array}$} & \multicolumn{3}{|c|}{$\begin{array}{c}\text { TOC subclínico, } \\
\text { ausência de outros TM } \\
(\mathrm{N}=\mathbf{3 1 7})\end{array}$} & \multicolumn{3}{|c|}{$\begin{array}{l}\text { TOC clínico } \\
\qquad(\mathrm{N}=77)\end{array}$} \\
\hline & \multirow{2}{*}{ Média } & \multicolumn{2}{|c|}{$95 \%$ IC } & \multirow{2}{*}{ Média } & \multicolumn{2}{|c|}{$95 \%$ IC } & \multirow{2}{*}{ Média } & \multicolumn{2}{|c|}{$95 \%$ IC } & \multirow{2}{*}{ Média } & \multicolumn{2}{|c|}{$95 \%$ IC } \\
\hline & & Inferior & Superior & & Inferior & Superior & & Inferior & Superior & & Inferior & Superior \\
\hline Saúde & 277,51 & 175,06 & 391,06 & $1.248,84$ & 938,26 & 1601,11 & 253,77 & 129,71 & 408,79 & $1.993,30$ & 644,19 & $3.800,27$ \\
\hline Medicamento & 8,02 & 4,00 & 12,84 & 97,13 & 56,21 & 146,72 & 9,39 & 2,39 & 18,67 & 114,96 & 12,36 & 283,00 \\
\hline Consulta médica & 7,86 & 4,33 & 12,22 & 83,45 & 53,13 & 119,52 & 17,02 & 3,52 & 38,75 & 74,18 & 14,21 & 177,53 \\
\hline Psicoterapia & 260,24 & 167,30 & 377,77 & $1.068,25$ & 801,91 & 1371,98 & 227,37 & 118,54 & 353,17 & $1.710,58$ & 537,11 & $3.563,71$ \\
\hline Serviço social & 326,17 & 125,47 & 554,32 & 220,60 & 16,76 & 533,32 & 772,84 & 199.69 & $1.570,04$ & 25,66 & 13,42 & 39,59 \\
\hline Assistente social & 1,24 & 0,93 & 1,56 & 3,36 & 2,55 & 4,15 & 1,54 & 0,84 & 2,37 & 5,09 & 2,56 & 8,03 \\
\hline Conselho tutelar & 0,44 & 0,31 & 0,58 & 1,27 & 0,95 & 1,63 & 0,43 & 0,16 & 0,75 & 1,33 & 0,41 & 2,40 \\
\hline Medida socioeducativa & 319,48 & 119,81 & 561,63 & 202,07 & 96,84 & 530,83 & 764,93 & 184,26 & 1607,26 & 0,00 & 0,00 & 0,00 \\
\hline Problemas escolares & 476,89 & 412,40 & 539,94 & $1.032,23$ & 871,49 & $1.200,56$ & 568,89 & 422,88 & 726,96 & $1.146,89$ & 698,96 & $1.736,21$ \\
\hline Repetência & 404,42 & 348,21 & 457,66 & 852,81 & 731,77 & 974,11 & 511,11 & 374,65 & 640,46 & 855,23 & 555,76 & $1.201,59$ \\
\hline Suspensão & 12,94 & 2,72 & 24,82 & 31,05 & 6,39 & 60,98 & 22,25 & 3,42 & 57,82 & 4,55 & 0,61 & 10,57 \\
\hline Evasão & 59,53 & 30,45 & 93,31 & 148,37 & 81,78 & 232,05 & 35,51 & 11,11 & 97,60 & 287,12 & 48,50 & 613,96 \\
\hline Perda de produtividade, pais & 73,25 & 61,39 & 89,83 & 147,64 & 105,66 & 196,69 & 62,25 & 51,02 & 75,77 & 146,78 & 37,08 & 319,11 \\
\hline TOTAL & $1.148,81$ & 904,35 & $1.440,48$ & $2.635,41$ & $2.142,16$ & $3.159,01$ & $1.651,81$ & 954,80 & $2.565,50$ & $3.293,38$ & $1.694,36$ & $5.370,71$ \\
\hline
\end{tabular}

$\mathrm{PPP}=$ power purchase parity, $\mathrm{IC}=$ intervalo de confiança, $\mathrm{TOC}=$ transtorno obsessivo-compulsivo 
Tabela 6. Custo nacional ao longo da vida do TOC subclínico e clínico.

\begin{tabular}{lcc}
\hline \multirow{2}{*}{ Tipo de custo } & \multicolumn{2}{c}{ Estimativa do custo nacional (US\$ PPP bilhão) } \\
\cline { 2 - 3 } & TOC subclínico & TOC clínico \\
\hline Saúde & 1,03 & 1,22 \\
Serviços sociais & 3,14 & 0,02 \\
Problemas escolares & 2,31 & 0,7 \\
Perda de produtividade parental & 0,25 & 0,09 \\
\hline TOTAL & 6,71 & 2,02 \\
\hline
\end{tabular}

Figura 3. Distribuição da estimativa do custo nacional do TOC.

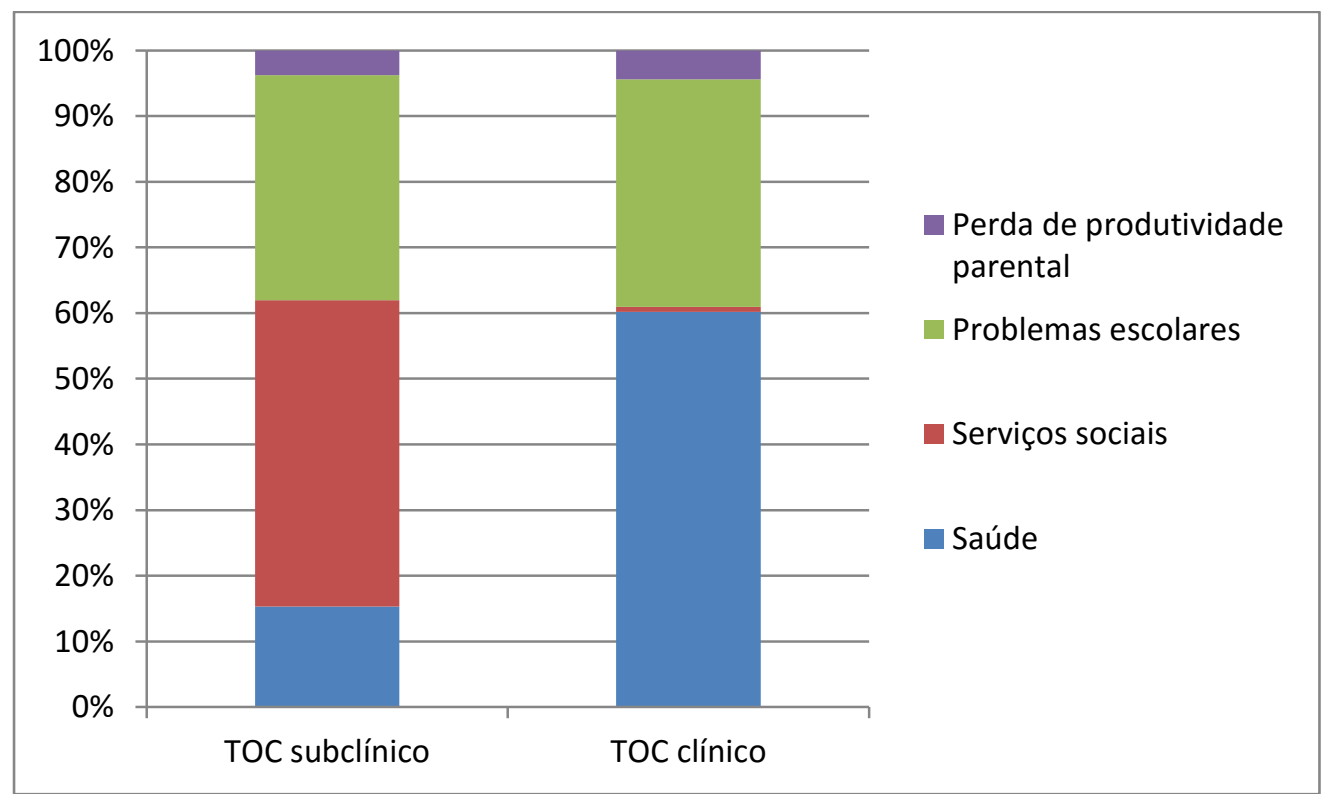




\section{DISCUSSÃO}

\subsection{Discussão: Estudo I e II}

A presente tese de doutorado procurou estimar a média do custo do transtorno mental na infância em nível subclínico e clínico e, por conseguinte, estimar o custo total destes para o Brasil. Este é o primeiro estudo que demonstrou o impacto econômico do transtorno mental subclínico na infância, dessa forma, torna-se impossível comparar com outros estudos.

Os dados demonstraram que o setor de saúde carrega a maior parte dos custos em ambas as análises de transtorno mentais clínicos (Estudo I e II). Um estudo analisou os custos dos transtornos de ansiedade em crianças (não incluiu diagnóstico de TOC) de amostra clínica também detectou que os cuidados em saúde geravam a maior parte dos custos na amostra (54). No entanto, outras pesquisas nos últimos anos têm apontado que o setor da educação carrega a maior parte dos custos dos transtornos mentais na infância. Uma revisão sistemática sobre estudos de custo da doença em transtorno de déficit de atenção de hiperatividade (TDAH) identificou custos da educação por criança na faixa de \$2.222-\$12.447, enquanto o custo para o setor de saúde foi \$621-\$2.720 (44). Uma revisão similar anterior já havia demonstrado este padrão (45), e outros estudos recentes também $(98,124)$. O mesmo padrão de dados foi encontrado em outros transtornos mentais na infância, como transtornos emocionais (98), transtornos de oposiçãodesafiante (46) e autismo (43). 
Importante ressaltar que na amostra total $10,5 \%$ se utilizaram de serviços de saúde. Somente $25,2 \%$ das crianças com transtorno mental clínico e 9,6\% das crianças com transtorno mental subclínico receberam algum tratamento em saúde mental (Estudo I). Apenas 18,2\% das crianças com TOC clínico e 8,5\% das crianças com TOC subclínico receberam algum tratamento em saúde mental (Estudo II). Ou seja, o número de crianças que precisavam de tratamento em saúde mental foi baixo em ambos os desfechos. A literatura especializada nesse tema aponta taxas de tratamento entre crianças e adolescentes com transtorno mental entre $16 \%$ e $58 \%$ (125), sendo que as taxas acima de 50\% foram apresentadas em países desenvolvidos. Um estudo epidemiológico conduzido nos Estados Unidos com amostragem representativa revelou uma taxa de tratamento especializado para crianças e adolescentes (3-17 anos) de apenas $20 \%$ (126). Um estudo epidemiológico brasileiro, conduzido em quatro regiões do país, apontou que 19,3\% das crianças e adolescentes com transtornos mentais não receberam nenhum tratamento especializado (101), corroborando com os dados da presente tese. Dessa forma, se as crianças com transtornos mentais recebessem tratamento de forma adequada, o custo da saúde seria ainda maior. Então, é importante destacar que o custo da saúde apresentado pelo presente trabalho está abaixo do que seria esperado, já que apenas uma pequena parcela das crianças brasileiras com transtornos mentais obtém tratamento de forma adequada.

É importante salientar que a maioria dos estudos que analisaram os custos educacionais se utilizou de perspectiva diferente do presente estudo. Usualmente, estes custos são relacionados a utilização de serviços de educação especial, enquanto no presente estudo o custo da educação foi derivado de problemas escolares (evasão escolar, suspensão e repetência), sendo assim, custos indiretos. Outros autores se utilizaram desse mesmo 
método em estudos sobre TDAH, transtorno de conduta $(46,127,128)$ e doenças transmissíveis (129). Considerando que os serviços de educação especial no Brasil são escassos, isto é, nem todas as escolas oferecem atendimentos desta natureza para crianças com transtornos mentais, se os custos da educação especial tivessem sido incluídos, provavelmente não alterariam os resultados de forma significativa.

Os custos do setor da educação representaram $42,2 \%$ e $36,7 \%$ do custo total de ter qualquer transtorno mental subclínico e clínico, ou $\$ 8,47$ bilhões; e $34,4 \%$ e $34,8 \%$ do custo total do TOC subclínico e clínico, ambos respectivamente. Este dado representa uma medida quantitativa dos problemas escolares para a sociedade, ou seja, o custo da repetição, suspensão e evasão escolar em crianças com transtornos mentais. Este achado pode ser explicado de algumas formas. Casos subclínicos podem ter menos chance de obter tratamento em razão de alguns fatores, como menor severidade sintomática e dificuldade parental em reconhecer os sintomas. Um estudo epidemiológico conduzido na Espanha descobriu que $60 \%$ das crianças com 8-12 anos de idade com TOC subclínico tiveram algum contato com serviços de saúde de mental, comparado com 85\% das crianças com TOC clínico (14). Assim, a falta de tratamento do transtorno mental subclínico pode gerar consequências negativas, como mostra o alto custo dos problemas escolares.

A associação entre saúde e escolaridade é bem estabelecida na literatura. Escolarização no início da vida é um fator importante para desfechos na vida adulta, como emprego e estado de saúde $(35,36,130,131)$. Há evidencia de que aspectos positivos, como características de personalidade e emoções, em escolares, podem reduzir o efeito dos transtornos mentais sobre problemas escolares (aprendizado e performance) (105). 
Dessa forma, é fundamental que a evasão e repetência escolar se tornem alvos de políticas públicas. Os cuidados em saúde mental da infância não devem se restringir ao setor de saúde. Escolas podem exercer um papel importante na detecção e prevenção dos transtornos mentais na infância, assim, a assistência comunitária em saúde mental pode trabalhar em conjunto com o sistema educacional $(95,132)$. Felizmente, atualmente há evidência de programas focados na identificação de casos $(133,134)$ e prevenção de evasão escolar (135). Programas como estes podem ser de grande utilidade se implementados em larga escala no Brasil.

Os dois estudos aqui apresentados foram os primeiros a quantificar o custo dos transtornos mentais subclínicos na infância. Os resultados demonstraram que a média do custo ao longo da vida de ter qualquer transtorno mental subclínico por criança foi de $\$ 1.750,86$, com custo nacional estimado em $\$ 9,92$ bilhões. Enquanto para o TOC subclínico a média do custo ao longo da vida foi $\$ 1.651,81$, somando um custo nacional estimado em $\$ 6,71$ bilhões. Estudos prévios já demonstraram que ter um transtorno mental subclínico está associado a prejuízo funcional de moderado a severo (136), qualidade de vida reduzida (9) e risco de suicídio (137). Consequentemente, pode-se argumentar que crianças com transtorno mental em nível subclínico poderiam se beneficiar de tratamento, especialmente em razão do alto risco do desenvolvimento de transtorno mental clínico (13).

Os altos custos do TOC subclínico demonstrados pelo presente estudo adicionam ao já crescente conjunto de evidências acerca dos efeitos deletérios dos transtornos mentais em nível subclínico. Um estudo longitudinal avaliou crianças de 11 anos de idade até a idade adulta indicou que sujeitos sem TOC ou outro transtorno mental, mas com 
sintomas obsessivo-compulsivos apresentam prejuízo funcional significativo. Crianças que reportavam obsessões e compulsões tinham quase cinco vezes mais chance de preencher critério diagnóstico para TOC na idade adulta (11). Utilizando a amostra do presente estudo, Alvarenga e colaboradores também demonstraram que o TOC subclínico tem prejuízo significativo $(31,104)$. Estas evidências se alinham a noção atual de psicopatologia dimensional e desenvolvimental.

Assim, oferecer tratamento para crianças com TOC subclínico pode ser uma estratégia importante para reduzir o prejuízo funcional e reverter a tendência de desenvolvimento do TOC clínico. Contudo, infelizmente, não existem estudos relacionados à prevenção e tratamento de TOC subclínico, entretanto a literatura apresenta uma gama de programas para prevenir outros transtornos mentais, como transtornos de ansiedade (138), depressão (139), psicoses (140), transtorno alimentar (141). Futuros estudos poderiam se embasar nas robustas evidências científicas acerca de fatores de risco e modelos teóricos, como da teoria cognitivo-comportamental, para desenvolver programas que visem prevenir o TOC clínico ou tratar o TOC subclínico.

A estimativa do custo nacional do TOC clínico foi $\$ 2,02$ bilhões. Até o presente momento, nenhum estudo verificou o custo do TOC na faixa etária da infância/adolescência. No entanto, um estudo publicado na década de 1990 estimou que o custo do TOC anual em adultos era de 8,4 bilhões de dólares (correção pela inflação, período 1990-2015: U\$15,3 bilhões) (142). Outro estudo, também analisando a faixa etária de adultos, estimou o custo do TOC na Europa em €2.27 bilhões (PPP) (143). Contudo, é difícil realizar comparações com dados derivados de amostras de adultos em razão da teoria do capital humano, isto é, a perda de produtividade no trabalho (custo 
indireto) em adultos não são diretamente comparáveis com os custos na faixa etária da infância. Além disso, seria razoável supor que os custos indiretos na idade adulta seriam maiores do que na infância/adolescência. Além disso, chama atenção o TOC clínico apresentar média de custo total acima da categoria outros transtornos mentais (\$3.293,38 vs. \$2.635,41). Isto pode ser devido ao padrão usual de início precoce, cronicidade e demora em receber diagnóstico e tratamento devido ao aspecto secreto do TOC.

Os dados apresentados revelam a sobrecarga (burden) dos transtornos mentais na infância e podem contribuir para discussões acerca de planejamento de políticas e orçamento. Avaliar os custos dos transtornos mentais pode ajudar a priorizar futuras avaliações econômicas, especialmente estudos de custo-efetividade, assim como identificar componentes e tendências dos custos (144). Além disso, pode ainda prover informações sobre a magnitude de um transtorno mental em particular gerando subsídios para maior conscientização da sociedade e gestores. Mais ainda, pode também adicionar elementos para o debate extremamente atual sobre planejamento de política e orçamento no Brasil e outros países em situações financeiras problemáticas.

Em vista disso, é importante ressaltar a alta prevalência dos transtornos mentais na infância e adolescência $(100,145)$ e que $60 \%$ das crianças não recebem nenhum tratamento especializado no Brasil $(101,146)$. Neste cenário, cortes de orçamento que afetem os setores da educação e da saúde podem se provar graves erros, levando a problemas ainda mais graves no futuro, como maior custo econômico para a sociedade, indivíduos e famílias. Gastos na área da saúde e educação devem ser entendidos como 
investimentos com o intuito de gerar desenvolvimento e evitar futuros gastos (147), principalmente quando os recursos são alocados de forma racional.

Neste sentido, um estudo recente de custo-utilidade (um tipo de avaliação de custoefetividade que tem como desfecho qualidade de vida e tempo de sobrevivência) indicou que a razão de custo-efetividade de tratar uma criança/adolescente com TDAH com metilfenidato no Brasil é superior a taxas de países de alta renda (148). Outro estudo demonstrou que a disponibilização de tratamento adequado para crianças e adolescentes com TDAH tem o potencial de economizar R $\$ 1,16$ bilhões por ano (149). Estas evidências de estudos brasileiros confirmam que é possível reduzir o impacto econômico dos transtornos mentais na infância com planejamento adequado e uso de dados científicos robustos. Além disso, vale ressaltar que nos últimos anos estudos internacionais têm apresentado dados importantes de custo-efetividade de tratamentos de transtornos mentais na infância e adolescência $(96,97,150)$, muitos dos quais poderiam embasar o planejamento dos serviços de saúde no Brasil com as devidas adequações culturais. No entanto, há uma marcada escassez de estudos de custoefetividade e custo-utilidade na literatura internacional (151).

\subsection{Limitações: Estudo I e II}

Os resultados apresentados precisam ser considerados em vista de algumas limitações. Primeiro, estudos de custo da doença não permitem determinar o uso eficiente de recursos ou o quanto se poderia economizar usando os dados apresentados $(80,90)$. Gestores necessitam de dados de custo-efetividade, custo-utilidade ou custo-benefício para definir que programas ou intervenções podem otimizar os recursos disponíveis. 
Segundo, não foram coletadas informações sobre outros potenciais custos, como educação especial e cuidado informal. Terceiro, alguns componentes de custos foram indiretamente estimados (como perda de produtividade dos pais e frequência de uso de serviços sociais), logo, alguns aspectos podem não representar a realidade de forma acurada.

Quarto, devido a distribuição não-normal dos dados de custos e tamanho da amostra, intervalos de confiança se mostraram grandes em diversas variáveis, algo que demonstra incerteza estatística. Quinto, a categoria de transtorno mental subclínico foi baseada nos algoritmos das bandas do instrumento DAWBA, ao invés do julgamento clínico, contudo, o uso do algoritmo oferece a vantagem de ser um método padronizado e reprodutível. Sexto, o critério de definição do TOC subclínico foi baseado apenas na sintomatologia, ainda que não exista um consenso sobre este tópico, alguns estudos se utilizaram de critérios diferentes, o que pode prejudicar futuras comparações e generalizações acerca dos dados. Sétimo, a estimativa do custo nacional foi baseada na generalização de dados de duas grandes cidades brasileiras que não representam o país como um todo. Diferença entre cidades e Estados podem influenciar os custos dos transtornos mentais. Por exemplo, a proporção de crianças com transtornos mentais que obtém tratamento pode ser menor em regiões em que o sistema de saúde é menos estruturado ou possui menor investimento público. Logo, há necessidade de cautela na interpretação destes dados específicos. Oitavo, os dados aqui apresentados são derivados de um estudo transversal, logo, a perspectiva se restringe a apenas um período no tempo. Uma perspectiva longitudinal poderia fornecer dados mais fidedignos, considerando a natureza crônica e dinâmica dos transtornos mentais na infância. 
Futuramente, com as novas avaliações do estudo HRC, novas análises poderão ser conduzidas com o intuito de adicionar um ponto de vista temporal aos dados.

\subsection{Considerações finais}

Em suma, os dados apresentados na presente tese de doutorado fornecem evidências de que transtornos mentais subclínicos e clínicos na infância tem um grande impacto econômico na sociedade, especialmente nos setores da saúde e educação. O conhecimento acerca do impacto econômico dos transtornos mentais na infância pode informar gestores e políticos sobre a magnitude do problema, de forma que seja possível planejar um sistema efetivo de cuidados com programas de tratamento e prevenção que sejam custo-efetivos. Em vista dos altos custos dos problemas escolares é essencial combater a evasão e repetência escolar com programas de prevenção precoce. Finalmente, recomenda-se que o governo evite cortar recursos dos setores da saúde e educação no Brasil, já que isto pode prejudicar a atual assistência às crianças e, possivelmente, aumentar ainda mais o impacto econômico dos transtornos mentais na infância no futuro próximo. 


\section{CONCLUSÕES}

- O transtorno mental infantil subclínico e clínico apresentaram médias de custo total ao longo da vida de $\$ 1.750,86$ e $\$ 3.141,21$, respectivamente (todos os valores em PPP).

- O custo nacional estimado do transtorno mental subclínico foi de \$9,92 bilhões, enquanto do transtorno mental clínico foi de \$11,65 bilhões.

- O TOC subclínico e clínico apresentaram médias de custo total ao longo da vida de $\$ 1.651,81$ e $\$ 3.293,38$, respectivamente.

- O custo nacional do TOC subclínico foi de $\$ 6,71$ bilhões, enquanto do TOC clínico foi de \$2,02 bilhões. 


\section{REFERÊNCIAS}

1. Collins PY, Patel V, Joestl SS, March D, Insel TR, Daar AS, et al. Grand challenges in global mental health. Nature. 2011 Jul 7;475(7354):27-30.

2. Prince M, Patel V, Saxena S, Maj M, Maselko J, Phillips MR, et al. No health without mental health. The Lancet. 2007 Sep;370(9590):859-77.

3. Vos T, Flaxman AD, Naghavi M, Lozano R, Michaud C, Ezzati M, et al. Years lived with disability (YLDs) for 1160 sequelae of 289 diseases and injuries 19902010: a systematic analysis for the Global Burden of Disease Study 2010. The Lancet. 2012 Dec 15;380(9859):2163-96.

4. Polanczyk GV, Salum GA, Sugaya LS, Caye A, Rohde LA. Annual research review: A meta-analysis of the worldwide prevalence of mental disorders in children and adolescents. J Child Psychol Psychiatry. 2015 Mar;56(3):345-65.

5. Kim-Cohen J, Caspi A, Moffitt TE, Harrington H, Milne BJ, Poulton R. Prior juvenile diagnoses in adults with mental disorder: developmental follow-back of a prospective-longitudinal cohort. Arch Gen Psychiatry. 2003 Jul;60(7):709-17.

6. Kessler RC, Angermeyer M, Anthony JC, DE Graaf R, Demyttenaere K, Gasquet I, et al. Lifetime prevalence and age-of-onset distributions of mental disorders in the World Health Organization's World Mental Health Survey Initiative. World Psychiatry Off J World Psychiatr Assoc WPA. 2007 Oct;6(3):168-76.

7. González-Tejera G, Canino G, Ramírez R, Chávez L, Shrout P, Bird H, et al. Examining minor and major depression in adolescents. J Child Psychol Psychiatry. 2005 Aug;46(8):888-99.

8. Scahill L, Schwab-Stone M, Merikangas KR, Leckman JF, Zhang H, Kasl S. Psychosocial and clinical correlates of ADHD in a community sample of schoolage children. J Am Acad Child Adolesc Psychiatry. 1999 Aug;38(8):976-84.

9. Bussing R, Mason DM, Bell L, Porter P, Garvan C. Adolescent outcomes of childhood attention-deficit/hyperactivity disorder in a diverse community sample. J Am Acad Child Adolesc Psychiatry. 2010 Jun;49(6):595-605.

10. Fergusson DM, Horwood LJ, Ridder EM, Beautrais AL. Subthreshold depression in adolescence and mental health outcomes in adulthood. Arch Gen Psychiatry. 2005 Jan;62(1):66-72.

11. Fullana MA, Mataix-Cols D, Caspi A, Harrington H, Grisham JR, Moffitt TE, et al. Obsessions and compulsions in the community: prevalence, interference, helpseeking, developmental stability, and co-occurring psychiatric conditions. Am J Psychiatry. 2009 Mar;166(3):329-36. 
12. Hill RM, Pettit JW, Lewinsohn PM, Seeley JR, Klein DN. Escalation to Major Depressive Disorder among adolescents with subthreshold depressive symptoms: evidence of distinct subgroups at risk. J Affect Disord. 2014 Apr;158:133-8.

13. Shankman SA, Lewinsohn PM, Klein DN, Small JW, Seeley JR, Altman SE. Subthreshold conditions as precursors for full syndrome disorders: a 15-year longitudinal study of multiple diagnostic classes. J Child Psychol Psychiatry. 2009 Dec;50(12):1485-94.

14. Canals J, Hernández-Martínez C, Cosi S, Voltas N. The epidemiology of obsessive-compulsive disorder in Spanish school children. J Anxiety Disord. 2012 Oct;26(7):746-52.

15. Vivan A de S, Rodrigues L, Wendt G, Bicca MG, Braga DT, Cordioli AV. Obsessive-compulsive symptoms and obsessive-compulsive disorder in adolescents: a population-based study. Rev Bras Psiquiatr São Paulo Braz 1999. 2014 Jun;36(2):111-8.

16. Balázs J, Keresztény A. Subthreshold attention deficit hyperactivity in children and adolescents: a systematic review. Eur Child Adolesc Psychiatry. 2014 Jun;23(6):393-408.

17. Bertha EA, Balázs J. Subthreshold depression in adolescence: a systematic review. Eur Child Adolesc Psychiatry. 2013 Oct;22(10):589-603.

18. Correll CU, Hauser M, Penzner JB, Auther AM, Kafantaris V, Saito E, et al. Type and duration of subsyndromal symptoms in youth with bipolar I disorder prior to their first manic episode. Bipolar Disord. 2014 Aug;16(5):478-92.

19. Valleni-Basile LA, Garrison CZ, Waller JL, Addy CL, McKeown RE, Jackson $\mathrm{KL}$, et al. Incidence of obsessive-compulsive disorder in a community sample of young adolescents. J Am Acad Child Adolesc Psychiatry. 1996;35(7):898-906.

20. Zammit S, Kounali D, Cannon M, David AS, Gunnell D, Heron J, et al. Psychotic experiences and psychotic disorders at age 18 in relation to psychotic experiences at age 12 in a longitudinal population-based cohort study. Am J Psychiatry. 2013 Jul;170(7):742-50.

21. American Psychiatric Association. Diagnostic And Statistical Manual Of Mental Disorders DSM-IV-TR Fourth Edition (Text Revision). 2000.

22. American Psychiatric Association. Diagnostic and Statistical Manual of Mental Disorders, 5th Edition: DSM-5. 5 edition. Washington, DC: American Psychiatric Publishing; 2013. 991 p.

23. Copeland W, Shanahan L, Costello EJ, Angold A. Cumulative prevalence of psychiatric disorders by young adulthood: a prospective cohort analysis from the Great Smoky Mountains Study. J Am Acad Child Adolesc Psychiatry. 2011 Mar;50(3):252-61. 
24. Taylor E, Rutter M. Classification. In: Rutter M, Bishop DVM, Pine D, Scott S, Stevenson J, Taylor E, et al., editors. Rutter's Child and Adolescent Psychiatry. Blackwell Publishing Ltd.; 2008.

25. Pincus HA, McQueen LE, Elinson L. Subthreshold Mental Disorders. In: Phillips KA, First HA, Pincus HA, editors. Advancing DSM Dillemas in Psychiatric Diagnosis. Washington, DC: American Psychiatric Association; 2002.

26. Coghill D, Sonuga-Barke EJS. Annual research review: categories versus dimensions in the classification and conceptualisation of child and adolescent mental disorders--implications of recent empirical study. J Child Psychol Psychiatry. 2012 May;53(5):469-89.

27. van Os J, Delespaul P, Wigman J, Myin-Germeys I, Wichers M. Psychiatry beyond labels: introducing contextual precision diagnosis across stages of psychopathology. Psychol Med. 2013 Jul;43(7):1563-7.

28. van Os J, Lataster T, Delespaul P, Wichers M, Myin-Germeys I. Evidence that a psychopathology interactome has diagnostic value, predicting clinical needs: an experience sampling study. PloS One. 2014;9(1):e86652.

29. Kessler RC, Berglund P, Demler O, Jin R, Merikangas KR, Walters EE. Lifetime prevalence and age-of-onset distributions of DSM-IV disorders in the National Comorbidity Survey Replication. Arch Gen Psychiatry. 2005 Jun;62(6):593-602.

30. van Os J. The Dynamics of Subthreshold Psychopathology: Implications for Diagnosis and Treatment. Am J Psychiatry. 2013 Jul 1;170(7):695-8.

31. Alvarenga PG, Cesar RC, Leckman JF, Moriyama TS, Torres AR, Bloch MH, et al. Obsessive-compulsive symptom dimensions in a population-based, crosssectional sample of school-aged children. J Psychiatr Res. 2015 Mar;62:108-14.

32. Sawyer MG, Whaiter L, Rey JM, Hazell PL, Graetz BW, Baghurst P. HealthRelated Quality of Life of Children and Adolescents With Mental Disorders. J Am Acad Child Adolesc Psychiatry. 2002 May;41(5):530-7.

33. Schieve LA, Blumberg SJ, Rice C, Visser SN, Boyle C. The Relationship Between Autism and Parenting Stress. PEDIATRICS. 2007 Feb 1;119(Supplement):S114-21.

34. Barry TD, Lyman RD, Klinger LG. Academic Underachievement and AttentionDeficit/Hyperactivity Disorder: The Negative Impact of Symptom Severity on School Performance. J Sch Psychol. 2002 May;40(3):259-83.

35. Barbaresi WJ, Katusic SK, Colligan RC, Weaver AL, Jacobsen SJ. Long-term school outcomes for children with attention-deficit/hyperactivity disorder: a population-based perspective. J Dev Behav Pediatr JDBP. 2007 Aug;28(4):26573. 
36. Tramontina S, Martins S, Michalowski MB, Ketzer CR, Eizirik M, Biederman J, et al. School dropout and conduct disorder in Brazilian elementary school students. Can J Psychiatry Rev Can Psychiatr. 2001 Dec;46(10):941-7.

37. Tramontina S, Martins S, Michalowski MB, Ketzer CR, Eizirik M, Biederman J, et al. Estimated mental retardation and school dropout in a sample of students from state public schools in Porto Alegre, Brazil. Rev Bras Psiquiatr. 2002 Oct;24(4):177-81.

38. Eaton WW, Martins SS, Nestadt G, Bienvenu OJ, Clarke D, Alexandre P. The burden of mental disorders. Epidemiol Rev. 2008;30:1-14.

39. Alonso J, Petukhova M, Vilagut G, Chatterji S, Heeringa S, Üstün TB, et al. Days out of role due to common physical and mental conditions: results from the WHO World Mental Health surveys. Mol Psychiatry. 2011 Dec;16(12):1234-46.

40. Levinson D, Lakoma MD, Petukhova M, Schoenbaum M, Zaslavsky AM, Angermeyer M, et al. Associations of serious mental illness with earnings: results from the WHO World Mental Health surveys. Br J Psychiatry. 2010 Aug $1 ; 197(2): 114-21$.

41. McCrone P. Mental health economics: current methodological issues. Epidemiol Psychiatr Sci. 2011 Sep;20(3):239-43.

42. Järbrink K, Knapp M. The economic impact of autism in Britain. Autism Int J Res Pract. 2001 Mar;5(1):7-22.

43. Knapp M, Romeo R, Beecham J. Economic cost of autism in the UK. Autism. 2009 May 1;13(3):317-36.

44. Doshi JA, Hodgkins P, Kahle J, Sikirica V, Cangelosi MJ, Setyawan J, et al. Economic impact of childhood and adult attention-deficit/hyperactivity disorder in the United States. J Am Acad Child Adolesc Psychiatry. 2012 Oct;51(10):990 1002.e2.

45. Pelham WE, Foster EM, Robb JA. The Economic Impact of AttentionDeficit/Hyperactivity Disorder in Children and Adolescents. J Pediatr Psychol. 2007 Jul 1;32(6):711-27.

46. Foster EM, Jones DE. The High Costs of Aggression: Public Expenditures Resulting From Conduct Disorder. Am J Public Health. 2005 Oct;95(10):176772.

47. Knapp M, Scott S, Davies J. The Cost of Antisocial Behaviour in Younger Children. Clin Child Psychol Psychiatry. 1999 Oct 1;4(4):457-73.

48. Romeo R, Knapp M, Scott S. Economic cost of severe antisocial behaviour in children--and who pays it. Br J Psychiatry J Ment Sci. 2006 Jun;188:547-53. 
49. Dusetzina SB, Farley JF, Weinberger M, Gaynes BN, Sleath B, Hansen RA. Treatment use and costs among privately insured youths with diagnoses of bipolar disorder. Psychiatr Serv Wash DC. 2012 Oct;63(10):1019-25.

50. Jin $\mathrm{H}, \mathrm{McC}$ rone P. Cost-of-illness studies for bipolar disorder: systematic review of international studies. PharmacoEconomics. 2015 Apr;33(4):341-53.

51. Domino ME, Burns BJ, Mario J, Reinecke MA, Vitiello B, Weller EB, et al. Service use and costs of care for depressed adolescents: who uses and who pays? J Clin Child Adolesc Psychol Off J Soc Clin Child Adolesc Psychol Am Psychol Assoc Div 53. 2009 Nov;38(6):826-36.

52. Knapp M, McCRONE P, Fombonne E, Beecham J, Wostear G. The Maudsley long-term follow-up of child and adolescent depression 3. Impact of comorbid conduct disorder on service use and costs in adulthood. Br J Psychiatry. 2002 Jan 1;180(1):19-23.

53. McCrone P, Knapp M, Fombonne E. The Maudsley long-term follow-up of child and adolescent depression. Eur Child Adolesc Psychiatry. 2005 Oct;14(7):40713.

54. Bodden DHM, Dirksen CD, Bögels SM. Societal Burden of Clinically Anxious Youth Referred for Treatment: A Cost-of-illness Study. J Abnorm Child Psychol. 2008 May;36(4):487-97.

55. Pro Bono Economics, BEAT. Costs of eating disorders in England: Economic impacts of anorexia nervosa, bulimia nervosa and other disorders, focussing on young people [Internet]. 2012. Available from:

http://www.probonoeconomics.com/sites/probonoeconomics.com/files/files/repor ts/PBE\%20BEAT\%20report\%20180512_0.pdf

56. UNICEF. The state of world's children 2015 [Internet]. UNICEF; 2014. Available from: www.unicef.org

57. Torres AR, Prince MJ, Bebbington PE, Bhugra D, Brugha TS, Farrell M, et al. Obsessive-compulsive disorder: prevalence, comorbidity, impact, and helpseeking in the British National Psychiatric Morbidity Survey of 2000. Am J Psychiatry. 2006 Nov;163(11):1978-85.

58. Kessler RC, Chiu WT, Demler O, Merikangas KR, Walters EE. Prevalence, severity, and comorbidity of 12-month DSM-IV disorders in the National Comorbidity Survey Replication. Arch Gen Psychiatry. 2005 Jun;62(6):617-27.

59. Rapoport JL, Inoff-Germain G, Weissman MM, Greenwald S, Narrow WE, Jensen PS, et al. Childhood obsessive-compulsive disorder in the NIMH MECA study: parent versus child identification of cases. Methods for the Epidemiology of Child and Adolescent Mental Disorders. J Anxiety Disord. 2000 Dec;14(6):535-48.

60. Geller D, Biederman J, Jones J, Park K, Schwartz S, Shapiro S, et al. Is juvenile obsessive-compulsive disorder a developmental subtype of the disorder? A 
review of the pediatric literature. J Am Acad Child Adolesc Psychiatry. 1998;37(4):420-7.

61. Swedo SE, Rapoport JL, Leonard H, Lenane M, Cheslow D. Obsessivecompulsive disorder in children and adolescents. Clinical phenomenology of 70 consecutive cases. Arch Gen Psychiatry. 1989 Apr;46(4):335-41.

62. Lack CW, Storch EA, Keeley ML, Geffken GR, Ricketts ED, Murphy TK, et al. Quality of life in children and adolescents with obsessive-compulsive disorder: base rates, parent-child agreement, and clinical correlates. Soc Psychiatry Psychiatr Epidemiol. 2009 Nov;44(11):935-42.

63. Stewart SE, Geller DA, Jenike M, Pauls D, Shaw D, Mullin B, et al. Long-term outcome of pediatric obsessive-compulsive disorder: a meta-analysis and qualitative review of the literature. Acta Psychiatr Scand. 2004 Jul;110(1):4-13.

64. Bloch MH, Craiglow BG, Landeros-Weisenberger A, Dombrowski PA, Panza KE, Peterson BS, et al. Predictors of Early Adult Outcomes in Pediatric-Onset Obsessive-Compulsive Disorder. Pediatrics. 2009 Oct 1;124(4):1085-93.

65. Do Rosario-Campos MC, Leckman JF, Mercadante MT, Shavitt RG, Da Silva Prado H, Sada P, et al. Adults with early-onset obsessive-compulsive disorder. Am J Psychiatry. 2001;158(11):1899-903.

66. Chabane N, Delorme R, Millet B, Mouren M-C, Leboyer M, Pauls D. Early-onset obsessive-compulsive disorder: a subgroup with a specific clinical and familial pattern? J Child Psychol Psychiatry. 2005 Aug;46(8):881-7.

67. de Mathis MA, Diniz JB, Shavitt RG, Torres AR, Ferrão YA, Fossaluza V, et al. Early onset obsessive-compulsive disorder with and without tics. CNS Spectr. $2009 \mathrm{Jul} ; 14(7): 362-70$.

68. Flessner CA, Allgair A, Garcia A, Freeman J, Sapyta J, Franklin ME, et al. The impact of neuropsychological functioning on treatment outcome in pediatric obsessive-compulsive disorder. Depress Anxiety. 2010 Apr;27(4):365-71.

69. Palermo SD, Bloch MH, Craiglow B, Landeros-Weisenberger A, Dombrowski PA, Panza K, et al. Predictors of early adulthood quality of life in children with obsessive-compulsive disorder. Soc Psychiatry Psychiatr Epidemiol. 2011 Apr;46(4):291-7.

70. Rapoport JL, Philip Shaw. Obsessive-Compulsive Disorder. In: Rutter's Child and Adolescent Psychiatry. 2008.

71. Freeman JB, Choate-Summers ML, Moore PS, Garcia AM, Sapyta JJ, Leonard HL, et al. Cognitive Behavioral Treatment for Young Children With ObsessiveCompulsive Disorder. Biol Psychiatry. 2007 Feb 1;61(3):337-43.

72. O'Kearney RT, Anstey KJ, von Sanden C. Behavioural and cognitive behavioural therapy for obsessive compulsive disorder in children and adolescents. Cochrane Database Syst Rev. 2006;(4):CD004856. 
73. Watson HJ, Rees CS. Meta-analysis of randomized, controlled treatment trials for pediatric obsessive-compulsive disorder. J Child Psychol Psychiatry. 2008 May;49(5):489-98.

74. Foa EB. Cognitive behavioral therapy of obsessive-compulsive disorder. Dialogues Clin Neurosci. 2010 Jun;12(2):199-207.

75. Barrett P, Healy-Farrell L, March JS. Cognitive-behavioral family treatment of childhood obsessive-compulsive disorder: a controlled trial. J Am Acad Child Adolesc Psychiatry. 2004 Jan;43(1):46-62.

76. O'Leary EMM, Barrett P, Fjermestad KW. Cognitive-behavioral family treatment for childhood obsessive-compulsive disorder: a 7-year follow-up study. J Anxiety Disord. 2009 Oct;23(7):973-8.

77. Asbahr FR, Castillo AR, Ito LM, Latorre MRD de O, Moreira MN, Lotufo-Neto F. Group cognitive-behavioral therapy versus sertraline for the treatment of children and adolescents with obsessive-compulsive disorder. J Am Acad Child Adolesc Psychiatry. 2005 Nov;44(11):1128-36.

78. Martin JL, Thienemann M. Group cognitive-behavior therapy with family involvement for middle-school-age children with obsessive-compulsive disorder: a pilot study. Child Psychiatry Hum Dev. 2005;36(1):113-27.

79. Thienemann M, Martin J, Cregger B, Thompson HB, Dyer-Friedman J. ManualDriven group cognitive-behavioral therapy for adolescents with obsessivecompulsive disorder: a pilot study. J Am Acad Child Adolesc Psychiatry. 2001 Nov;40(11):1254-60.

80. Byford S, Torgerson DJ, Raftery J. Economic note: cost of illness studies. BMJ. 2000 May 13;320(7245):1335.

81. Hsia RY, Belfer ML. A framework for the economic analysis of child and adolescent mental disorders. Int Rev Psychiatry Abingdon Engl. 2008 Jun;20(3):251-9.

82. Rice DP. Estimating the cost of illness. Am J Public Health Nations Health. 1967 Mar;57(3):424-40.

83. Cooper BS, Rice DP. The economic cost of illness revisited. Soc Secur Bull. 1976 Feb;39(2):21-36.

84. Hodgson TA, Meiners MR. Cost-of-illness methodology: a guide to current practices and procedures. Milbank Mem Fund Q Health Soc. 1982;60(3):429-62.

85. Rice DP, Kelman S, Miller LS. The economic burden of mental illness. Hosp Community Psychiatry. 1992 Dec;43(12):1227-32.

86. Rice D. Cost of illness studies: what is good about them? Inj Prev. 2000 Sep;6(3):177-9. 
87. Brouwer WBF, van Exel NJA, Koopmanschap MA, Rutten FFH. Productivity costs before and after absence from work: as important as common? Health Policy Amst Neth. 2002 Aug;61(2):173-87.

88. Rice D. Cost-of-illness studies: fact or fiction? The Lancet. 1994 Dec;344(8936):1519-20.

89. Tarricone R. Cost-of-illness analysis: What room in health economics? Health Policy. 2006 Jun;77(1):51-63.

90. Akobundu E, Ju J, Blatt L, Mullins CD. Cost-of-illness studies : a review of current methods. PharmacoEconomics. 2006;24(9):869-90.

91. Raftery J. Costing in economic evaluation. BMJ. 2000 Jun 10;320(7249):1597.

92. Cunnama L, Sinanovic E, Ramma L, Foster N, Berrie L, Stevens W, et al. Using Top-down and Bottom-up Costing Approaches in LMICs: The Case for Using Both to Assess the Incremental Costs of New Technologies at Scale. Health Econ. 2016 Feb 1;25:53-66.

93. Chapko MK, Liu C-F, Perkins M, Li Y-F, Fortney JC, Maciejewski ML. Equivalence of two healthcare costing methods: bottom-up and top-down. Health Econ. 2009 Oct;18(10):1188-201.

94. Glied S, Cuellar AE. Trends And Issues In Child And Adolescent Mental Health. Health Aff (Millwood). 2003 Sep 1;22(5):39-50.

95. P.D. D, Couto MCV. Intersetorialidade: uma exigência da clínica com crianças na Atenção Psicossocial. In: Atenção em Saúde Mental para crianças e adolescentes no SUS. Hucitec; 2010. p. 271-179.

96. Byford S, Barrett B, Roberts C, Wilkinson P, Dubicka B, Kelvin RG, et al. Costeffectiveness of selective serotonin reuptake inhibitors and routine specialist care with and without cognitive-behavioural therapy in adolescents with major depression. Br J Psychiatry. 2007 Dec 1;191(6):521-7.

97. Domino ME, Burns BJ, Silva SG, Kratochvil CJ, Vitiello B, Reinecke MA, et al. Cost-effectiveness of treatments for adolescent depression: results from TADS. Am J Psychiatry. 2008 May;165(5):588-96.

98. Snell T, Knapp M, Healey A, Guglani S, Evans-Lacko S, Fernandez J-L, et al. Economic impact of childhood psychiatric disorder on public sector services in Britain: estimates from national survey data. J Child Psychol Psychiatry. 2013 Sep;54(9):977-85.

99. Knapp M, Mangalore R, Simon J. The global costs of schizophrenia. Schizophr Bull. 2004;30(2):279-93.

100. Fleitlich-Bilyk B, Goodman R. Prevalence of child and adolescent psychiatric disorders in southeast Brazil. J Am Acad Child Adolesc Psychiatry. 2004 Jun;43(6):727-34. 
101. Paula CS, Bordin IAS, Mari JJ, Velasque L, Rohde LA, Coutinho ESF. The Mental Health Care Gap among Children and Adolescents: Data from an Epidemiological Survey from Four Brazilian Regions. PLoS ONE. 2014 Feb 18;9(2):e88241.

102. Weissman MM, Wickramaratne P, Adams P, Wolk S, Verdeli H, Olfson M. Brief screening for family psychiatric history: the family history screen. Arch Gen Psychiatry. 2000 Jul;57(7):675-82.

103. Milne BJ, Moffitt TE, Crump R, Poulton R, Rutter M, Sears MR, et al. How should we construct psychiatric family history scores? A comparison of alternative approaches from the Dunedin Family Health History Study. Psychol Med. 2008 Dec;38(12):1793-802.

104. Alvarenga PG, Rosario MC do, Cesar RC, Manfro GG, Moriyama TS, Bloch $\mathrm{MH}$, et al. Obsessive-compulsive symptoms are associated with psychiatric comorbidities, behavioral and clinical problems: a population-based study of Brazilian school children. Eur Child Adolesc Psychiatry. 2015 May 27;25(2):175-82.

105. Hoffmann MS, Leibenluft E, Stringaris A, Laporte PP, Pan PM, Gadelha A, et al. Positive Attributes Buffer the Negative Associations Between Low Intelligence and High Psychopathology With Educational Outcomes. J Am Acad Child Adolesc Psychiatry. 2016 Jan 1;55(1):47-53.

106. Krieger FV, Polanczyk GV, Goodman R, Rohde LA, Graeff-Martins AS, Salum $\mathrm{G}$, et al. Dimensions of Oppositionality in a Brazilian Community Sample: Testing the DSM-5 Proposal and Etiological Links. J Am Acad Child Adolesc Psychiatry [Internet]. 2013 Apr [cited 2014 Oct 19];52(4). Available from: http://www.ncbi.nlm.nih.gov/pmc/articles/PMC3834546/

107. Mogg K, Salum GA, Bradley BP, Gadelha A, Pan P, Alvarenga P, et al. Attention network functioning in children with anxiety disorders, attentiondeficit/hyperactivity disorder and non-clinical anxiety. Psychol Med. 2015 Sep;45(12):2633-46.

108. Pan PM, Salum GA, Gadelha A, Moriyama T, Cogo-Moreira H, Graeff-Martins AS, et al. Manic symptoms in youth: dimensions, latent classes, and associations with parental psychopathology. J Am Acad Child Adolesc Psychiatry. 2014 Jun;53(6):625-34.e2.

109. Salum GA, Mogg K, Bradley BP, Gadelha A, Pan P, Tamanaha AC, et al. Threat bias in attention orienting: evidence of specificity in a large community-based study. Psychol Med. 2013 Apr;43(4):733-45.

110. Salum GA, Sergeant J, Sonuga-Barke E, Vandekerckhove J, Gadelha A, Pan PM, et al. Specificity of basic information processing and inhibitory control in attention deficit hyperactivity disorder. Psychol Med. 2014 Feb;44(3):617-31.

111. Sato JR, Salum GA, Gadelha A, Vieira G, Zugman A, Picon FA, et al. Decreased centrality of subcortical regions during the transition to adolescence: A functional 
connectivity study. NeuroImage [Internet]. 2014 [cited 2014 Oct 14]; Available from: http://www.sciencedirect.com/science/article/pii/S1053811914008052

112. Sato JR, Biazoli CE, Salum GA, Gadelha A, Crossley N, Satterthwaite TD, et al. Temporal stability of network centrality in control and default mode networks: Specific associations with externalizing psychopathology in children and adolescents. Hum Brain Mapp. 2015 Dec;36(12):4926-37.

113. Wagner F, Martel MM, Cogo-Moreira H, Maia CRM, Pan PM, Rohde LA, et al. Attention-deficit/hyperactivity disorder dimensionality: the reliable " $\mathrm{g}$ " and the elusive "s" dimensions. Eur Child Adolesc Psychiatry. 2016 Jan;25(1):83-90.

114. Goodman R, Ford T, Richards H, Gatward R, Meltzer H. The Development and Well-Being Assessment: description and initial validation of an integrated assessment of child and adolescent psychopathology. J Child Psychol Psychiatry. $2000 \mathrm{Jul} ; 41(5): 645-55$.

115. Goodman A, Heiervang E, Collishaw S, Goodman R. The "DAWBA bands" as an ordered-categorical measure of child mental health: description and validation in British and Norwegian samples. Soc Psychiatry Psychiatr Epidemiol. 2011 Jun;46(6):521-32.

116. Alvarenga PG. Sintomas obsessivo-compulsivos em escolares: prevalência, dimensões psicopatológicas, agregação familiar, comorbidades e fatores clínicos associados [Internet] [Tese de Doutorado]. Universidade de São Paulo; 2014 [cited 2014 Oct 30]. Available from: http://www.teses.usp.br/teses/disponiveis/5/5142/tde-04082014-084337/

117. Austin PC. An Introduction to Propensity Score Methods for Reducing the Effects of Confounding in Observational Studies. Multivar Behav Res. 2011 May 31;46(3):399-424.

118. Drummond MF, Sculpher MJ, Torrance GW, O’Brien BJ, Stoddart GL. Methods for the Economic Evaluation of Health Care Programmes. 3 edition. Oxford; New York: Oxford University Press; 2005. 396 p.

119. IBGE. Índice Nacional de Preços ao Consumidor Amplo (IPCA) [Internet]. 2015. Available from:

http://www.ibge.gov.br/home/estatistica/indicadores/precos/inpc_ipca/ipcainpc_201509_1.shtm

120. Gray AM, Clarke PM, Wolstenholme JL, Wordsworth S. Defining, measuring, and valuing costs. In: Applied methods of cost-effectiveness analysis in health care. Oxford University Press; 2011. (Handbooks in health economic evaluation).

121. The World Bank. World Development Indicators, Price level ratio of PPP conversion factor (GDP) to market exchange rate [Internet]. 2015 [cited 2015 Oct 28]. Available from: http://data.worldbank.org/indicator/PA.NUS.PPPC.RF 
122. Gray AM, Clarke PM, Wolstenholme JL, Wordsworth S. Chapter 7 Analysing cost. In: Applied Methods of Cost-effectiveness Analysis in Healthcare. 1 edition. Oxford; New York: Oxford University Press; 2010.

123. IBGE. Censo Demográfico 2010, Caracteristicas da Populacao e dos Domicílios [Internet]. 2010. Available from:

ftp://ftp.ibge.gov.br/Censos/Censo_Demografico_2010/Resultados_do_Universo/ ods/Brasil/tab1_1_1.zip

124. Telford C, Green C, Logan S, Langley K, Thapar A, Ford T. Estimating the costs of ongoing care for adolescents with attention-deficit hyperactivity disorder. Soc Psychiatry Psychiatr Epidemiol. 2013 Feb;48(2):337-44.

125. Ford T. Practitioner review: How can epidemiology help us plan and deliver effective child and adolescent mental health services? J Child Psychol Psychiatry. 2008 Sep;49(9):900-14.

126. Kataoka SH, Zhang L, Wells KB. Unmet need for mental health care among U.S. children: variation by ethnicity and insurance status. Am J Psychiatry. 2002 Sep;159(9):1548-55.

127. Jones DE, Foster EM, Conduct Problems Prevention Research Group. Service use patterns for adolescents with ADHD and comorbid conduct disorder. J Behav Health Serv Res. 2009 Oct;36(4):436-49.

128. Robb JA, Sibley MH, Pelham WE, Foster EM, Molina BSG, Gnagy EM, et al. The Estimated Annual Cost of ADHD to the U.S. Education System. School Ment Health. 2011 Sep 1;3(3):169-77.

129. Suaya JA, Shepard DS, Siqueira JB, Martelli CT, Lum LCS, Tan LH, et al. Cost of Dengue Cases in Eight Countries in the Americas and Asia: A Prospective Study. Am J Trop Med Hyg. 2009 May 1;80(5):846-55.

130. Conti G, Heckman J, Urzua S. THE EDUCATION-HEALTH GRADIENT. Am Econ Rev. 2010 May;100(2):234-8.

131. Kessler RC, Foster CL, Saunders WB, Stang PE. Social consequences of psychiatric disorders, I: Educational attainment. Am J Psychiatry. 1995 Jul;152(7):1026-32.

132. Couto MCV, Duarte CS, Delgado PGG. Child mental health and Public Health in Brazil: current situation and challenges. Rev Bras Psiquiatr. 2008 Dec;30(4):3849.

133. Celina Andrade Pereira CLW. A randomised controlled trial of a web-based educational program in child mental health for schoolteachers. Eur Child Amp Adolesc Psychiatry. 2014;

134. Vieira MA, Gadelha AA, Moriyama TS, Bressan RA, Bordin IA. Evaluating the effectiveness of a training program that builds teachers' capability to identify and 
appropriately refer middle and high school students with mental health problems in Brazil: an exploratory study. BMC Public Health. 2014 Feb 28;14(1):210.

135. Graeff-Martins AS, Oswald S, Comassetto JO, Kieling C, Gonçalves RR, Rohde LA, et al. A package of interventions to reduce school dropout in public schools in a developing country. A feasibility study. Eur Child Adolesc Psychiatry. 2006 Dec;15(8):442-9.

136. Roberts RE, Fisher PW, Turner JB, Tang M. Estimating the burden of psychiatric disorders in adolescence: the impact of subthreshold disorders. Soc Psychiatry Psychiatr Epidemiol. 2015 Mar;50(3):397-406.

137. Balázs J, Miklósi M, Keresztény Á, Hoven CW, Carli V, Wasserman C, et al. Adolescent subthreshold-depression and anxiety: psychopathology, functional impairment and increased suicide risk. J Child Psychol Psychiatry. 2013 Jun 1;54(6):670-7.

138. Jr BJF, Richard D, Mann A. The Prevention of Child and Adolescent Anxiety: A Meta-analytic Review. Prev Sci. 2011 Mar 25;12(3):255-68.

139. Horowitz JL, Garber J. The prevention of depressive symptoms in children and adolescents: A meta-analytic review. J Consult Clin Psychol. 2006 Jun;74(3):401-15.

140. Stafford MR, Jackson H, Mayo-Wilson E, Morrison AP, Kendall T. Early interventions to prevent psychosis: systematic review and meta-analysis. BMJ. 2013 Jan 18;346(jan18 1):f185-f185.

141. Stice E, Shaw H, Marti CN. A meta-analytic review of eating disorder prevention programs: encouraging findings. Annu Rev Clin Psychol. 2007;3:207-31.

142. DuPont RL, Rice DP, Shiraki S, Rowland CR. Economic costs of obsessivecompulsive disorder. Med Interface. 1995 Apr;8(4):102-9.

143. Olesen J, Gustavsson A, Svensson M, Wittchen H-U, Jönsson B, CDBE2010 study group, et al. The economic cost of brain disorders in Europe. Eur J Neurol. 2012 Jan;19(1):155-62.

144. Koopmanschap MA. Cost-of-illness studies. Useful for health policy? PharmacoEconomics. 1998 Aug;14(2):143-8.

145. Paula CS, Coutinho ES, Mari JJ, Rohde LA, Miguel EC, Bordin IA, et al. Prevalence of psychiatric disorders among children and adolescents from four Brazilian regions. Rev Bras Psiquiatr. 2015 Jun;37(2):178-9.

146. Fatori D, Evans-Lacko S, de Paula C. Child mental health care in Brazil: barriers and achievements. The Lancet. 2012;379(9812):e16-7.

147. Knapp M. Mental health in an age of austerity. Evid Based Ment Health. 2012 Aug 1;15(3):54-5. 
148. Maia CR, Stella SF, Wagner F, Pianca TG, Krieger FV, Cruz LN, et al. Costutility analysis of methylphenidate treatment for children and adolescents with ADHD in Brazil. Rev Bras Psiquiatr Sao Paulo Braz 1999. 2015 Sep 8;

149. Maia CR, Stella SF, Mattos P, Polanczyk GV, Polanczyk CA, Rohde LA. The Brazilian policy of withholding treatment for ADHD is probably increasing health and social costs. Rev Bras Psiquiatr São Paulo Braz 1999. 2015 Mar;37(1):67-70.

150. O’Neill D, McGilloway S, Donnelly M, Bywater T, Kelly P. A cost-effectiveness analysis of the Incredible Years parenting programme in reducing childhood health inequalities. Eur J Health Econ HEPAC Health Econ Prev Care. 2013 Feb;14(1):85-94.

151. Beecham J. Annual research review: Child and adolescent mental health interventions: a review of progress in economic studies across different disorders. J Child Psychol Psychiatry. 2014 Jun;55(6):714-32. 


\section{ANEXOS}

ANEXO A. Tabela da distribuição dos problemas escolares, uso de serviços sociais e uso de tratamentos de saúde por transtorno mental na infância $(\mathrm{N}=2512)$.

\begin{tabular}{|c|c|c|c|}
\hline & & nstorno Men & \\
\hline & Ausência & Subclínico & Clínico \\
\hline Problemas escolares & $\mathbf{N}(\%)$ & $\mathbf{N}(\%)$ & $\mathbf{N}(\%)$ \\
\hline Repetência escola & & & \\
\hline Sim & $241(15,4 \%)$ & $111(21,7 \%)$ & $145(33,6 \%)$ \\
\hline Não & $1326(84,5 \%)$ & $400(78,3 \%)$ & $286(66,2 \%)$ \\
\hline Não sabe & $2(0,1 \%)$ & 0 & $1(0,2 \%)$ \\
\hline Abandono escola & & & \\
\hline Sim & $17(1,1 \%)$ & $15(2,9 \%)$ & $20(4,6 \%)$ \\
\hline Não & $1549(98,7 \%)$ & $496(97,1 \%)$ & $410(94,9 \%)$ \\
\hline Não sabe & $3(0,2 \%)$ & 0 & $2(0,5 \%)$ \\
\hline Suspensão escola & & & \\
\hline Sim & $54(3,4 \%)$ & $20(3,9 \%)$ & $42(9,7 \%)$ \\
\hline Não & $1515(96,6 \%)$ & $491(96,1 \%)$ & $390(90,3 \%)$ \\
\hline Serviços sociais & & & \\
\hline Assistente social & & & \\
\hline Sim & $53(3,4 \%)$ & $36(7,0 \%)$ & $54(12,5 \%)$ \\
\hline Não & $1499(95,5 \%)$ & $466(91,2 \%)$ & $374(86,6 \%)$ \\
\hline Não sabe & $17(1,1 \%)$ & $9(1,8 \%)$ & $4(0,9 \%)$ \\
\hline Conselho tutelar & & & \\
\hline Sim & $37(2,4 \%)$ & $24(4,7 \%)$ & $37(8,6 \%)$ \\
\hline Não & $1490(95 \%)$ & $475(93 \%)$ & $385(89,1 \%)$ \\
\hline Não sabe & $42(2,7 \%)$ & $12(2,3 \%)$ & $10(2,3 \%)$ \\
\hline Medida socioeduc & & & \\
\hline Sim & $12(0,8 \%)$ & $1(0,2 \%)$ & $1(0,2 \%)$ \\
\hline Não & $1550(98,8 \%)$ & $505(98,8 \%)$ & $429(99,3 \%)$ \\
\hline Não sabe & $7(0,4 \%)$ & $5(1 \%)$ & $2(0,5 \%)$ \\
\hline Saúde & & & \\
\hline Medicamento & & & \\
\hline Sim & $42(2,7 \%$ & $26(5,1 \%)$ & $59(13,7 \%)$ \\
\hline Não & $1526(97,3 \%)$ & $485(94,9 \%)$ & $373(86,3 \%)$ \\
\hline Não sabe & $1(0,1 \%)$ & 0 & 0 \\
\hline Psicoterapia & & & \\
\hline $\operatorname{Sim}$ & $64(4,1 \%)$ & $50(9,8 \%)$ & $96(22,2 \%)$ \\
\hline Não & $1504(95,9 \%)$ & $461(90,2 \%)$ & $336(77,8 \%)$ \\
\hline Não sabe & $1(0,1 \%)$ & 0 & 0 \\
\hline Hospitalização & & & \\
\hline Sim & $1(0,1 \%)$ & 0 & 0 \\
\hline Não & $1568(99,9 \%)$ & $511(100 \%)$ & $432(100 \%$ \\
\hline
\end{tabular}


ANEXO B. Tabela da distribuição dos problemas escolares, uso de serviços sociais e uso de tratamentos de saúde por TOC subclínico e clínico, e outros transtornos mentais na infância $(\mathrm{N}=2512)$.

\begin{tabular}{|c|c|c|c|c|}
\hline & $\begin{array}{c}\text { Ausência } \\
\text { de TM }\end{array}$ & Outros TMs & $\begin{array}{c}\text { TOC } \\
\text { subclínico, } \\
\text { ausência de } \\
\text { outros TMs }\end{array}$ & $\begin{array}{c}\text { TOC } \\
\text { clínico }\end{array}$ \\
\hline Problemas escolares & $\mathbf{N}(\%)$ & $\mathbf{N}(\%)$ & $\mathbf{N}(\%)$ & $\mathbf{N}(\%)$ \\
\hline \multicolumn{5}{|l|}{ Repetência escolar } \\
\hline Sim & $235(15,5 \%)$ & $179(29,8 \%)$ & $59(18,6 \%)$ & $24(31,2 \%)$ \\
\hline Não & $1281(84,4 \%)$ & $420(70 \%)$ & $258(81,4 \%)$ & $53(68,8 \%)$ \\
\hline Não sabe & $2(0,1 \%)$ & $1(0,2 \%)$ & 0 & 0 \\
\hline \multicolumn{5}{|l|}{ Abandono escolar } \\
\hline Sim & $21(1,4 \%)$ & $24(4 \%)$ & $2(0,6 \%)$ & $5(6,5 \%)$ \\
\hline Não & $1494(98,4 \%)$ & $575(95,8 \%)$ & $315(99,4 \%)$ & $71(92,2 \%)$ \\
\hline Não sabe & $3(0,2 \%)$ & $1(0,2 \%)$ & 0 & $1(1,3 \%)$ \\
\hline \multicolumn{5}{|l|}{ Suspensão escolar } \\
\hline Sim & $52(3,4 \%)$ & $39(6,5 \%)$ & $22(6,9 \%)$ & $3(3,9 \%)$ \\
\hline Não & $1466(96,6 \%)$ & $561(93,5 \%)$ & $295(93,1 \%)$ & $74(96,1 \%)$ \\
\hline \multicolumn{5}{|l|}{ Serviços sociais } \\
\hline \multicolumn{5}{|l|}{ Assistente social } \\
\hline Sim & $55(3,6 \%)$ & $61(10,2 \%)$ & $15(4,7 \%)$ & $12(15,6 \%)$ \\
\hline Não & $1442(95 \%)$ & $536(89,3 \%)$ & $297(93,7 \%)$ & $64(83,1 \%)$ \\
\hline Não sabe & $21(1,4 \%)$ & $3(0,5 \%)$ & $5(1,6 \%)$ & $1(1,3 \%)$ \\
\hline \multicolumn{5}{|l|}{ Conselho tutelar } \\
\hline Sim & $39(2,6 \%)$ & $45(7,5 \%)$ & $8(2,5 \%)$ & $6(7,8 \%)$ \\
\hline Não & $1435(94,5 \%)$ & $546(91 \%)$ & $300(94,6 \%)$ & $69(89,6 \%)$ \\
\hline Não sabe & $44(2,9 \%)$ & $9(1,5 \%)$ & $9(2,8 \%)$ & $2(2,6 \%)$ \\
\hline \multicolumn{5}{|c|}{ Medida socioeducativa } \\
\hline Sim & $8(0,5 \%)$ & $2(0,3 \%)$ & $4(1,3 \%)$ & 0 \\
\hline Não & $1502(98,9 \%)$ & $594(99 \%)$ & $312(98,4 \%)$ & $76(98,7 \%)$ \\
\hline Não sabe & $8(0,5 \%)$ & $4(0,7 \%)$ & $1(0,3 \%)$ & $1(1,3 \%)$ \\
\hline \multicolumn{5}{|l|}{ Saúde } \\
\hline \multicolumn{5}{|l|}{ Medicamento } \\
\hline Sim & $42(2,8 \%)$ & $67(11,2 \%)$ & $9(2,8 \%)$ & $9(11,7 \%)$ \\
\hline Não & $1475(97,2 \%)$ & $533(88,8 \%)$ & $308(97,2 \%)$ & $68(88,3 \%)$ \\
\hline Não sabe & $1(0,1 \%)$ & 0 & 0 & 0 \\
\hline \multicolumn{5}{|l|}{ Psicoterapia } \\
\hline Sim & $68(4,5 \%)$ & $108(18 \%)$ & $21(6,6 \%)$ & $13(16,9 \%)$ \\
\hline Não & $1450(95, \%)$ & $491(81,8 \%)$ & $491(81,8 \%)$ & $64(83,1 \%)$ \\
\hline Não sabe & 0 & $1(0,2 \%)$ & 0 & 0 \\
\hline \multicolumn{5}{|l|}{ Hospitalização } \\
\hline Sim & $1(0,1 \%)$ & 0 & 0 & 0 \\
\hline Não & $1517(99,9 \%)$ & $600(100 \%)$ & $317(100 \%)$ & $77(100 \%)$ \\
\hline
\end{tabular}

TM: transtorno mental, TOC: transtorno obsessivo-compulsivo 
ANEXO C. Artigo I submetido durante o período de doutoramento.

Title: The Economic Impact of Subthreshold and Clinical Childhood Mental Disorders in Brazil

Keywords: cost-of-illness, child, mental disorders, subthreshold mental disorders

Daniel Fatori, Giovanni Salum, Alex Itria, Pedro Pan, Pedro Alvarenga, Luis Augusto Rohde, Rodrigo Bressan, Ary Gadelha, Jair de Jesus Mari, Maria Conceição do Rosário, Gisele Manfro, Guilherme Polanczyk, Euripedes Constantino Miguel, Ana Soledade Graeff-Martins

\section{INTRODUCTION}

Mental disorders are common health problems, affecting $13.4 \%$ of children and adolescents in the world (1), causing serious impairment and deleterious effects in quality of life (2) and are frequently chronic (3). In 2013, mental disorders accounted for $10.1 \%$ of disability-adjusted life years in 5-14 years old children (4). Evidences suggest that only around half of children with mental disorders receive treatment $(5,6)$ and that the majority of countries are not prepared to tackle this demand (7). In addition to this, recently many countries have been facing low economic growth and even economic recession (8), demanding austerity measures like spending cuts in several sectors, such as education and health.

Also, some children present psychiatric symptoms in a level below the threshold for categorical diagnosis, however, they suffer impairment (9-11) and are at greater risk for developing a clinical mental disorder later in life $(12,13)$. Moreover, there is evidence that subthreshold mental disorder is associated with negative outcomes, such as grade retention (14). Thus, it may lead to immediate and long-term economic impact. Therefore, it is essential to understand the economic impact of childhood mental disorders to provide data that can show potential budget priorities. 
The objective of the present study is to estimate the costs of clinical and subthreshold mental disorders in a sample of 6-14 years old children living in Brazil.

\section{METHODS}

\section{Data source}

The High Risk Cohort Study (HRC) is a large community study conducted in two major Brazilian cities, Sao Paulo and Porto Alegre. Subjects were 6-14 years old children (at the screening interview) being registered at school by a biological parent primary caretaker. In the first stage, parents of all eligible subjects were invited to participate in the study and then assessed with the Family History Screening (FHS) (15). A total of 8,012 families were interviewed, providing information about 9,937 children. From this pool, two subgroups were further investigated using a random-selection $(n=958)$ and high-risk group selection procedure $(n=1,514)$, resulting in a total sample of 2,512 subjects. Detailed information about the HRC study can be found elsewhere (16). The ethics committee of the University of São Paulo approved the study. Written consent from parents of all participants, and verbal assent from all children were obtained.

\section{Assessment of mental disorders}

The Development and Well-Being Assessment (DAWBA) (17) is a well-known and widely used structured interview administered by trained lay interviewers developed to assess DSM mental disorders in children and adolescents. The validated Brazilian Portuguese version of the DAWBA (18) was administered to parents. For the present study, mental disorders were assigned using the DAWBA bands, an ordered-categorical measure generated via a computer-algorithm. The DAWBA bands inform the probability of a child presenting a positive diagnosis based on ratings of symptoms and/or impairment. A previous study showed a dose-response association between DAWBA bands and service use and risk/protective factors (19). We converted the 
original five probability categories into three categories: no mental disorders $(\sim 0.5 \%$ and $\sim 3 \%$ ), any subthreshold mental disorder $(\sim 15 \%)$ and any clinical mental disorder $(\sim 50 \%$ and $>70 \%)$.

\section{Service use and school problems}

A structured questionnaire was designed by HRC study researchers to measure health and social services use and school problems. Parents were asked about children's lifetime mental health service use (medication, psychotherapy and hospitalization), contact with social services (social worker, child protection and juvenile justice) and school problems (retention, suspension and dropout). Lifetime frequency and/or duration were asked for all variables, except for hospitalization, contact with social services and parental absenteeism. Estimates of frequency and/or duration were assumed for these variables.

\section{Cost estimation}

The present study considered the societal perspective in order to estimate costs, namely, the economic impact of mental disorders on society as a whole, including direct and indirect costs (20). All costs estimations were based on 2010 data (the year of data collection) in Brazilian Reais.

Costs were categorized into four groups:

1) Mental health costs, treatment: use of psychiatric medication, psychotherapy and hospitalization (frequency and duration). The cost of psychiatric medication was extracted from the Brazilian Health Surveillance Agency (ANVISA) and for every medication the lowest cost value and a minimum therapeutic dosage was applied. Number of contacts with medical doctors and psychologists were based on the duration of treatment (one contact every three months) and costs of contact with health professionals was estimated using standardized values from the Brazilian Medical Association (AMB) for medical doctors and the Federal Council of Psychology (CFP) for psychologists. The cost of hospitalization for mental disorders was valued using data 
from the Information Technology Department of the Public Health Care System (DATASUS). Duration and cost of hospitalization were derived from the average registered in DATASUS.

2) Social services costs: contact with child protection service, social workers and youth detention center (juvenile justice). Number of contacts with child protection service and social worker was based on a previous population-based study of children in Brazil that showed the average was 3 and 5, respectively (Fatori et al., unpublished data). Then, we applied costs using the average salary (social worker and child protection counselor) from the Ministry of Labor and Employment registry of employment data. To estimate duration and costs of incarceration in youth detention centers we requested information from the Sao Paulo State Government and they provided the average duration of incarceration (10 months) in youth detention centers and its annual cost.

3) School problems: school retention, dropout and suspension (number of times each of these events happened). We calculated the economic loss attributed to school absence (dropout and suspension) and retention (losing a year of school) as a product of the number of school days lost times the economic value of a public school day based on data from the National Fund of Education Development (FUNDEB). All children in the sample were enrolled in public schools.

4) Parental loss of productivity: we estimated the loss of productivity as an indirect cost of childhood mental disorders based on the human capital method (21), which means that the loss of productivity was valued as earnings lost. Loss of productivity was based on primary caregiver reported salary. Two hours of work absenteeism were attributed for each contact with health professionals and social services.

\section{Weighting procedure}

Due to the oversampling strategy in the sampling process, prevalence data was weighted. Variables derived from the FHS interview were used in a propensity score matching analysis, in which 2,371 subjects selected for participation in the study were matched to the 1,500 subjects randomly selected from the screened sample. The 
matching procedure significantly reduced difference between both samples. Propensity scores were used as weights to calculate prevalence data.

\section{Cost analysis}

First, costs were corrected for inflation (2010-2015 period) using the IPCA index (22). Then, costs were converted to purchasing power parity (PPP) considering the last available conversion factor (2014) in order to allow comparison of data across different countries. The PPP conversion factor is the number of units of a currency required to buy the same amount of goods and services in relation with the United States dollar (23).

We estimated the mean cost for all variables in US\$ PPP. Considering the non-normal distribution of cost data, as well as statistical uncertainty, 95\% confidence intervals and standard errors were generated using the bootstrapping method, that is, 1,000 replications of random sampling with replacement (24).

We generated the national estimate cost of childhood mental disorders (subthreshold and clinical) based on data from the last Brazilian population census conducted by the Brazilian Institute of Geography and Statistics (IBGE). Then, mean costs were multiplied by the estimated number of children with subthreshold and clinical mental disorder based on weighted prevalence data from the DAWBA bands.

\section{RESULTS}

The prevalence of child clinical mental disorders in the sample according to the DAWBA bands was $12 \cdot 7 \%(\mathrm{n}=268)$ and subthreshold mental disorders were present in $19.4 \%(n=409)$ of the sample $(53 \cdot 1 \%$ were males $)$. Table 1 presents lifetime mean costs (all costs in US\$ PPP) per child by mental disorder categories. Subthreshold and clinical mental disorder showed lifetime mean total cost of $\$ 1,750 \cdot 86$ and $\$ 3,141 \cdot 21$, respectively. Mean costs of having any subthreshold mental disorder were greater than not having any mental disorder. We found substantial differences between cost categories, for instance, mean health cost of having any clinical mental disorder is $9 \cdot 2$ 
times higher than having no mental disorder and the mean total cost of having clinical mental disorder was 2.7 times higher than having no mental disorder. Subthreshold mental disorder mean health cost was $4 \cdot 3$ times higher than having no mental disorder. Hospitalization costs were omitted due to lack of subjects (only one child was hospitalized).

The national lifetime cost estimate for the population of 6-14 years old children with clinical mental disorder in Brazil was $\$ 12.79$ billion, whereas for subthreshold mental disorder it was $\$ 10.82$ billion (table 2). Both categories amounted $\$ 23.61$ billion. The health sector accounted for $44 \%$ and $53 \%$ of the national costs of subthreshold and clinical mental disorder, respectively. School problems were the second largest contributor to subthreshold and clinical mental disorder total costs (figure 1).

\section{DISCUSSION}

This study sought to estimate the economic impact of childhood clinical and subthreshold mental disorders using data from a large Brazilian community school age sample. To our knowledge, this is the first study to quantify the cost of subthreshold mental disorders in children. Our results indicated that clinical and subthreshold mental disorders in children have a great economic impact on society, especially in the health and education sectors.

We found that the health sector bears most of the economic burden. A study analyzing the cost of anxiety disorders in referred children (25) also found direct health care to be the main contributor of total costs. However, most studies in recent years have shown that the education sector bears most of the economic burden of mental disorders in children. A systematic review of attention deficit and hyperactivity disorder (ADHD) cost-of-illness studies identified education costs in the range of $\$ 2,222-\$ 12,447$ per children, while healthcare was \$621-\$2,720 (US dollars) (26). Recent ADHD studies confirmed this same pattern $(27,28)$. Moreover, similar patterns have been described for emotional disorders (27), oppositional-defiant disorder (29) and autism (30). 
It is important to highlight that most studies analyzed education costs with a different perspective as compared to the present study. Usually, education costs are related to utilization of special education services, whereas in our study education costs were evaluated according to school absenteeism (grade retention, school suspension and evasion), considering that special education services are scarce in Brazil. Other authors used a similar approach in studies about ADHD, conduct disorder $(29,31)$, and communicable diseases (32). Considering that most public schools in Brazil either don't offer special education for children with mental disorders or they do not offer specialized treatment from mental health professionals, we would probably not find different results, if special education were included.

Education costs represented $42 \cdot 2 \%$ and $36.7 \%$ of total lifetime costs of clinical and subthreshold mental disorders, respectively, or $\$ 8.47$ billion. The association between health and schooling is well-known in the literature. Educational attainment in early life is an important factor for later life outcomes, such as employment and health status $(33,34)$. So, it is important to address the issue of grade retention and school evasion. Child mental health care should not be restricted to the healthcare sector. Schools can have an important role in the identification and prevention of mental disorders in children, hence, community mental health care should work in tandem with the educational system (35). Currently, there is evidence for programs focused on improving case identification (36) and school dropout prevention (37). Programs like these could prove useful if implemented in a large scale.

Our results showed that the lifetime mean cost of subthreshold mental disorder per child was $\$ 1,750 \cdot 86$, amounting to a national estimate of $\$ 9.92$ billion (versus $\$ 11.65$ billion for clinical disorders). Previous studies showed that having a subthreshold mental disorder is associated with moderate to severe impairment (38) and increased risk for suicide (39). Consequently, it could be argued that children with subthreshold mental disorder can benefit from early intervention programs, especially considering the known risk to develop full-blown mental disorder later in life (12). Despite the growing body of evidence showing programs to prevent depression (40), anxiety disorders (41), eating disorders (42) and psychosis (43) in children and adolescents there is still a lack of evidence for other mental disorders, such as ADHD and obsessive-compulsive disorder. 
More research is still needed to circumvent the problem of small to moderate effect sizes and methodological problems. Therefore, future studies should address the economic impact of early intervention for mental disorders. More importantly, our data is consistent with the concept of mental disorders as a continuum from mental health and resilience to mental disorders.

The present study can contribute to a discussion about policy and budget planning. Our data presented the societal burden of childhood mental disorders in Brazil. First and foremost, it is important to point out the high prevalence of mental disorders in children and adolescents and that more than $60 \%$ of children with mental disorders do not receive any specialized mental health treatment in Brazil $(44,45)$. In this scenario, cutting health and education budget resources could be a mistake, leading to worse problems in the future, like higher costs to society, individuals and families. Spending on health and education must be seen as an investment to generate future economic savings, especially when resources are allocated rationally and are based on sound scientific evidence.

Assessing the economic impact of any disease has important limitations. First, it is difficult to determine cost savings of preventing or eradicating a disease. Cost-of-illness studies, such as the present study, can help prioritize diseases for future economic evaluations, clarifying cost components and show trends in costs (47). Also, this type of data cannot inform decision makers about the efficiency of resources being used, nor how to reallocate resources (20). To better inform policy makers' cost-effectiveness studies must be considered. Child mental health cost-effectiveness studies are still scarce worldwide (48), especially in low and middle income countries, but there is evidence at the present moment to advise policy makers on how to spend resources optimally. A recent cost-utility study indicated that the cost-effectiveness ratio of treating children and adolescents with ADHD with methylphenidate in Brazil was better than in high-income countries (49). Another study showed that with adequate treatment for children and adolescents with ADHD, Brazil would save an estimated R\$ 1.16 billion per year (50). Hence, it is possible to reduce the costs of childhood mental disorders with careful planning and good scientific evidence. 
Our results, in particular, should be considered in light of some limitations. First, we did not gather information about other relevant potential costs, such as especial education and informal care. Second, some cost components were estimated based on a priori data or estimation based on a conservative approach (i.e. parental loss of productivity), not individual-level collected data, and thus it is possible that some aspects are not representative of reality. Third, due to non-normally distributed nature of cost data and sample size, confidence intervals were large across most variables showing statistical uncertainty. Fourth, the subthreshold mental disorder category was based on the DAWBA bands algorithm, instead of a pragmatic clinical approach based on evidence from the literature, although it has the advantage of being a standardized and reproducible method. Fifth, we estimated national costs via generalization of data from a sample of children in two major cities in Brazil, thus it is not representative of the country as a whole.

In summary, this study provides evidence that child subthreshold and clinical mental disorders have a great economic impact on society, especially in the health and education sector. Knowing the economic burden of mental disorders in children in Brazil can inform policy makers about the magnitude of the problem, so that it is possible to plan an effective system of care with cost-effective programs of treatment and prevention. In view of the high cost of grade retention and school evasion, it is essential to tackle this issue with early interventions to prevent school absenteeism. Finally, we recommend the government to avoid health and education budgets cuts in Brazil, since this could harm the assistance of children and, possibly, increase the already huge economic burden of childhood mental disorders. To face the huge economic burden of childhood mental disorders, we believe that low and middle income countries should increase its investments to make the education and health systems work together to implement cost-effective strategies focused in the early stages of mental disorders.

\section{Acknowledgments}

This work is supported by the National Institute of Developmental Psychiatry for Children and Adolescents, a science and technology institute funded by Conselho 
Nacional de Desenvolvimento Científico e Tecnológico (CNPq; National Council for Scientific and Technological Development; grant number 573974/2008-0) and Fundação de Amparo à Pesquisa do Estado de São Paulo (FAPESP; Research Support Foundation of the State of São Paulo; grant number 2008/57896-8).

\section{REFERENCES}

1. Polanczyk GV, Salum GA, Sugaya LS, Caye A, Rohde LA. Annual research review: A meta-analysis of the worldwide prevalence of mental disorders in children and adolescents. J Child Psychol Psychiatry. 2015 Mar;56(3):345-65.

2. Sawyer MG, Whaiter L, Rey JM, Hazell PL, Graetz BW, Baghurst P. Health-Related Quality of Life of Children and Adolescents With Mental Disorders. Journal of the American Academy of Child \& Adolescent Psychiatry. 2002 May;41(5):530-7.

3. Kim-Cohen J, Caspi A, Moffitt TE, Harrington H, Milne BJ, Poulton R. Prior juvenile diagnoses in adults with mental disorder: developmental follow-back of a prospectivelongitudinal cohort. Arch Gen Psychiatry. 2003 Jul;60(7):709-17.

4. Institute for Health Metrics and Evaluation (IHME). GBD Compare [Internet]. IHME. 2015 [cited 2015 Oct 29]. Available from: http://www.healthdata.org/datavisualization/gbd-compare

5. Kataoka SH, Zhang L, Wells KB. Unmet need for mental health care among U.S. children: variation by ethnicity and insurance status. Am J Psychiatry. 2002 Sep;159(9):1548-55.

6. Sturm R, Ringel JS, Andreyeva T. Geographic disparities in children's mental health care. Pediatrics. 2003 Oct;112(4):e308.

7. Shatkin JP, Belfer ML. The Global Absence of Child and Adolescent Mental Health Policy. Child and Adolescent Mental Health. 2004 Sep 1;9(3):104-8.

8. The World Bank. World Development Indicators, GDP growth (annual \%) [Internet]. 2015 [cited 2015 Oct 28]. Available from: http://data.worldbank.org/indicator/NY.GDP.MKTP.KD.ZG

9. Alvarenga PG, Cesar RC, Leckman JF, Moriyama TS, Torres AR, Bloch MH, et al. Obsessive-compulsive symptom dimensions in a population-based, cross-sectional sample of school-aged children. J Psychiatr Res. 2015 Mar;62:108-14.

10. González-Tejera G, Canino G, Ramírez R, Chávez L, Shrout P, Bird H, et al. Examining minor and major depression in adolescents. J Child Psychol Psychiatry. 2005 Aug;46(8):888-99.

11. Scahill L, Schwab-Stone M, Merikangas KR, Leckman JF, Zhang H, Kasl S. Psychosocial and clinical correlates of ADHD in a community sample of school-age children. J Am Acad Child Adolesc Psychiatry. 1999 Aug;38(8):976-84. 
12. Shankman SA, Lewinsohn PM, Klein DN, Small JW, Seeley JR, Altman SE.

Subthreshold conditions as precursors for full syndrome disorders: a 15-year longitudinal study of multiple diagnostic classes. J Child Psychol Psychiatry. 2009 Dec;50(12):148594.

13. Hill RM, Pettit JW, Lewinsohn PM, Seeley JR, Klein DN. Escalation to Major Depressive Disorder among adolescents with subthreshold depressive symptoms: evidence of distinct subgroups at risk. J Affect Disord. 2014 Apr;158:133-8.

14. Bussing R, Mason DM, Bell L, Porter P, Garvan C. Adolescent outcomes of childhood attention-deficit/hyperactivity disorder in a diverse community sample. J Am Acad Child Adolesc Psychiatry. 2010 Jun;49(6):595-605.

15. Weissman MM, Wickramaratne P, Adams P, Wolk S, Verdeli H, Olfson M. Brief screening for family psychiatric history: the family history screen. Arch Gen Psychiatry. $2000 \mathrm{Jul} ; 57(7): 675-82$.

16. Salum GA, Gadelha A, Pan PM, Moriyama TS, Graeff-Martins AS, Tamanaha AC, et al. High risk cohort study for psychiatric disorders in childhood: rationale, design, methods and preliminary results. Int J Methods Psychiatr Res. 2015 Mar 1;24(1):58-73.

17. Goodman R, Ford T, Richards H, Gatward R, Meltzer H. The Development and WellBeing Assessment: description and initial validation of an integrated assessment of child and adolescent psychopathology. J Child Psychol Psychiatry. 2000 Jul;41(5):645-55.

18. Fleitlich-Bilyk B, Goodman R. Prevalence of child and adolescent psychiatric disorders in southeast Brazil. J Am Acad Child Adolesc Psychiatry. 2004 Jun;43(6):727-34.

19. Goodman A, Heiervang E, Collishaw S, Goodman R. The "DAWBA bands" as an ordered-categorical measure of child mental health: description and validation in British and Norwegian samples. Soc Psychiatry Psychiatr Epidemiol. 2011 Jun;46(6):521-32.

20. Akobundu E, Ju J, Blatt L, Mullins CD. Cost-of-illness studies : a review of current methods. Pharmacoeconomics. 2006;24(9):869-90.

21. Drummond MF, Sculpher MJ, Torrance GW, O'Brien BJ, Stoddart GL. Cost-benefit analysis. In: Methods for the Economic Evaluation of Health Care Programmes. 3 edition. Oxford; New York: Oxford University Press; 2005.

22. IBGE. Índice Nacional de Preços ao Consumidor Amplo (IPCA) [Internet]. 2015. Available from:

http://www.ibge.gov.br/home/estatistica/indicadores/precos/inpc_ipca/ipcainpc_201509_1.shtm

23. The World Bank. World Development Indicators, Price level ratio of PPP conversion factor (GDP) to market exchange rate [Internet]. 2015 [cited 2015 Oct 28]. Available from: http://data.worldbank.org/indicator/PA.NUS.PPPC.RF

24. Gray AM, Clarke PM, Wolstenholme JL, Wordsworth S. Chapter 7 Analysing cost. In: Applied Methods of Cost-effectiveness Analysis in Healthcare. 1 edition. Oxford; New York: Oxford University Press; 2010. 
25. Bodden DHM, Dirksen CD, Bögels SM. Societal Burden of Clinically Anxious Youth Referred for Treatment: A Cost-of-illness Study. J Abnorm Child Psychol. 2008 May;36(4):487-97.

26. Doshi JA, Hodgkins P, Kahle J, Sikirica V, Cangelosi MJ, Setyawan J, et al. Economic impact of childhood and adult attention-deficit/hyperactivity disorder in the United States. J Am Acad Child Adolesc Psychiatry. 2012 Oct;51(10):990-1002.e2.

27. Snell T, Knapp M, Healey A, Guglani S, Evans-Lacko S, Fernandez J-L, et al. Economic impact of childhood psychiatric disorder on public sector services in Britain: estimates from national survey data. J Child Psychol Psychiatry. 2013 Sep;54(9):977-85.

28. Telford C, Green C, Logan S, Langley K, Thapar A, Ford T. Estimating the costs of ongoing care for adolescents with attention-deficit hyperactivity disorder. Soc Psychiatry Psychiatr Epidemiol. 2013 Feb;48(2):337-44.

29. Foster EM, Jones DE. The High Costs of Aggression: Public Expenditures Resulting From Conduct Disorder. Am J Public Health. 2005 Oct;95(10):1767-72.

30. Knapp M, Romeo R, Beecham J. Economic cost of autism in the UK. Autism. 2009 May 1;13(3):317-36.

31. Jones DE, Foster EM, Conduct Problems Prevention Research Group. Service use patterns for adolescents with ADHD and comorbid conduct disorder. J Behav Health Serv Res. 2009 Oct;36(4):436-49.

32. Suaya JA, Shepard DS, Siqueira JB, Martelli CT, Lum LCS, Tan LH, et al. Cost of Dengue Cases in Eight Countries in the Americas and Asia: A Prospective Study. Am J Trop Med Hyg. 2009 May 1;80(5):846-55.

33. Barbaresi WJ, Katusic SK, Colligan RC, Weaver AL, Jacobsen SJ. Long-term school outcomes for children with attention-deficit/hyperactivity disorder: a population-based perspective. J Dev Behav Pediatr. 2007 Aug;28(4):265-73.

34. Conti G, Heckman J, Urzua S. THE EDUCATION-HEALTH GRADIENT. Am Econ Rev. 2010 May;100(2):234-8.

35. Couto MCV, Duarte CS, Delgado PGG. Child mental health and Public Health in Brazil: current situation and challenges. Revista Brasileira de Psiquiatria. 2008 Dec;30(4):3849.

36. Vieira MA, Gadelha AA, Moriyama TS, Bressan RA, Bordin IA. Evaluating the effectiveness of a training program that builds teachers' capability to identify and appropriately refer middle and high school students with mental health problems in Brazil: an exploratory study. BMC Public Health. 2014 Feb 28;14(1):210.

37. Graeff-Martins AS, Oswald S, Comassetto JO, Kieling C, Gonçalves RR, Rohde LA, et al. A package of interventions to reduce school dropout in public schools in a developing country. A feasibility study. Eur Child Adolesc Psychiatry. 2006 Dec;15(8):442-9.

38. Roberts RE, Fisher PW, Turner JB, Tang M. Estimating the burden of psychiatric disorders in adolescence: the impact of subthreshold disorders. Soc Psychiatry Psychiatr Epidemiol. 2015 Mar;50(3):397-406. 
39. Balázs J, Miklósi M, Keresztény Á, Hoven CW, Carli V, Wasserman C, et al. Adolescent subthreshold-depression and anxiety: psychopathology, functional impairment and increased suicide risk. Journal of Child Psychology and Psychiatry. 2013 Jun 1;54(6):670-7.

40. Horowitz JL, Garber J. The prevention of depressive symptoms in children and adolescents: A meta-analytic review. J Consult Clin Psychol. 2006 Jun;74(3):401-15.

41. Jr BJF, Richard D, Mann A. The Prevention of Child and Adolescent Anxiety: A Metaanalytic Review. Prev Sci. 2011 Mar 25;12(3):255-68.

42. Stice E, Shaw H, Marti CN. A meta-analytic review of eating disorder prevention programs: encouraging findings. Annu Rev Clin Psychol. 2007;3:207-31.

43. Stafford MR, Jackson H, Mayo-Wilson E, Morrison AP, Kendall T. Early interventions to prevent psychosis: systematic review and meta-analysis. BMJ. 2013 Jan 18;346(jan18 1):f185-f185.

44. Fatori D, Evans-Lacko S, de Paula C. Child mental health care in Brazil: barriers and achievements. The Lancet. 2012;379(9812):e16-7.

45. Paula CS, Bordin IAS, Mari JJ, Velasque L, Rohde LA, Coutinho ESF. The Mental Health Care Gap among Children and Adolescents: Data from an Epidemiological Survey from Four Brazilian Regions. PLoS ONE. 2014 Feb 18;9(2):e88241.

46. Knapp M. Mental health in an age of austerity. Evid Based Mental Health. 2012 Aug $1 ; 15(3): 54-5$.

47. Koopmanschap MA. Cost-of-illness studies. Useful for health policy? Pharmacoeconomics. 1998 Aug;14(2):143-8.

48. Beecham J. Annual research review: Child and adolescent mental health interventions: a review of progress in economic studies across different disorders. J Child Psychol Psychiatry. 2014 Jun;55(6):714-32.

49. Maia CR, Stella SF, Wagner F, Pianca TG, Krieger FV, Cruz LN, et al. Cost-utility analysis of methylphenidate treatment for children and adolescents with ADHD in Brazil. Rev Bras Psiquiatr. 2015 Sep 8;

50. Maia CR, Stella SF, Mattos P, Polanczyk GV, Polanczyk CA, Rohde LA. The Brazilian policy of withholding treatment for ADHD is probably increasing health and social costs. Rev Bras Psiquiatr. 2015 Mar;37(1):67-70. 
Table 1. Lifetime mean costs (US\$ PPP) per child by mental disorders categories $(\mathrm{N}=\mathbf{2 5 1 2}$ ).

\begin{tabular}{|c|c|c|c|c|c|c|c|c|c|}
\hline & \multicolumn{3}{|c|}{ No mental disorder $(\mathrm{N}=1569)$} & \multicolumn{3}{|c|}{$\begin{array}{c}\text { Any subthreshold } \\
\text { mental disorder }(\mathrm{N}=\mathbf{5 1 1})\end{array}$} & \multicolumn{3}{|c|}{$\begin{array}{l}\text { Any clinical mental } \\
\text { disorder }(N=432)\end{array}$} \\
\hline & \multirow[b]{2}{*}{$\begin{array}{l}\text { Mean cost } \\
\text { per child }\end{array}$} & \multicolumn{2}{|c|}{$95 \% \mathrm{CI}$} & \multicolumn{3}{|c|}{$95 \% \mathrm{CI}$} & \multirow[b]{2}{*}{$\begin{array}{l}\text { Mean cost } \\
\text { per child }\end{array}$} & \multicolumn{2}{|c|}{$95 \% \mathrm{CI}$} \\
\hline & & Lower & Upper & $\begin{array}{l}\text { Mean cost } \\
\text { per child }\end{array}$ & Lower & Upper & & Lower & Upper \\
\hline Health & $180 \cdot 50$ & $111 \cdot 26$ & $255 \cdot 91$ & $777 \cdot 25$ & $467 \cdot 67$ & $1172 \cdot 49$ & $1676 \cdot 22$ & $1243 \cdot 30$ & $2154 \cdot 05$ \\
\hline Medication & $5 \cdot 08$ & $2 \cdot 57$ & $8 \cdot 02$ & $44 \cdot 08$ & $15 \cdot 25$ & $86 \cdot 20$ & $119 \cdot 85$ & $71 \cdot 76$ & $176 \cdot 12$ \\
\hline Consultation & $7 \cdot 80$ & $3 \cdot 76$ & $13 \cdot 87$ & $28 \cdot 32$ & $13 \cdot 23$ & $49 \cdot 37$ & $107 \cdot 39$ & $66 \cdot 95$ & $152 \cdot 90$ \\
\hline Psychotherapy & $166 \cdot 25$ & $107 \cdot 39$ & $241 \cdot 59$ & $704 \cdot 84$ & $416 \cdot 13$ & $1075 \cdot 18$ & 1448.98 & $1071 \cdot 58$ & $1859 \cdot 27$ \\
\hline Social Services & $469 \cdot 84$ & $235 \cdot 46$ & $741 \cdot 26$ & $131 \cdot 13$ & $9 \cdot 89$ & $381 \cdot 88$ & $162 \cdot 63$ & $17 \cdot 83$ & $474 \cdot 45$ \\
\hline Social Worker & $1 \cdot 15$ & $0 \cdot 85$ & $1 \cdot 45$ & $2 \cdot 32$ & $1 \cdot 57$ & $3 \cdot 08$ & $4 \cdot 13$ & $3 \cdot 08$ & $5 \cdot 20$ \\
\hline Child Protection & $0 \cdot 40$ & $0 \cdot 28$ & $0 \cdot 54$ & $0 \cdot 80$ & $0 \cdot 50$ & $1 \cdot 13$ & 1.45 & $0 \cdot 99$ & 1.95 \\
\hline Youth Detention & 463.63 & $230 \cdot 06$ & $718 \cdot 07$ & $118 \cdot 63$ & $110 \cdot 70$ & $469 \cdot 01$ & $140 \cdot 33$ & $130 \cdot 92$ & $546 \cdot 89$ \\
\hline School Problems & $468 \cdot 81$ & $409 \cdot 25$ & $534 \cdot 04$ & $739 \cdot 10$ & $598 \cdot 60$ & $885 \cdot 07$ & $1154 \cdot 32$ & $946 \cdot 07$ & $1355 \cdot 47$ \\
\hline Repetition & $411 \cdot 34$ & $362 \cdot 20$ & $464 \cdot 37$ & $608 \cdot 09$ & $499 \cdot 74$ & $727 \cdot 91$ & $919 \cdot 77$ & $777 \cdot 79$ & $1068 \cdot 45$ \\
\hline Suspension & $16 \cdot 02$ & $5 \cdot 33$ & $29 \cdot 25$ & $13 \cdot 04$ & $2 \cdot 34$ & $32 \cdot 48$ & $32 \cdot 15$ & $7 \cdot 15$ & $69 \cdot 22$ \\
\hline Dropout & $41 \cdot 45$ & $19 \cdot 02$ & $67 \cdot 83$ & $117 \cdot 97$ & $49 \cdot 35$ & $197 \cdot 14$ & $202 \cdot 40$ & $102 \cdot 35$ & $323 \cdot 14$ \\
\hline Parental Loss of Productivity & $65 \cdot 02$ & $55 \cdot 33$ & 79.79 & $112 \cdot 76$ & $77 \cdot 36$ & $174 \cdot 37$ & $164 \cdot 76$ & $120 \cdot 87$ & $215 \cdot 26$ \\
\hline TOTAL & $1179 \cdot 51$ & $914 \cdot 88$ & $1467 \cdot 57$ & $1750 \cdot 86$ & $1301 \cdot 89$ & $2236 \cdot 95$ & $3141 \cdot 21$ & $2592 \cdot 83$ & $3821 \cdot 21$ \\
\hline
\end{tabular}

$\mathrm{PPP}=$ power purchase parity, $\mathrm{CI}=$ confidence interval 
Table 2. Lifetime national cost estimate of childhood subthreshold and clinical mental disorders.

\begin{tabular}{|c|c|c|}
\hline \multirow[b]{2}{*}{ Cost type } & \multicolumn{2}{|c|}{ National cost estimate (US\$ PPP billion) } \\
\hline & $\begin{array}{c}\text { Subthreshold mental } \\
\text { disorder }\end{array}$ & $\begin{array}{l}\text { Clinical mental } \\
\text { disorder }\end{array}$ \\
\hline Health Costs & 4.4 & 6.22 \\
\hline Social Services Costs & 0.74 & 0.6 \\
\hline School Problems & 4.19 & 4.28 \\
\hline Parental Loss of Productivity & 0.64 & 0.61 \\
\hline TOTAL COSTS & 9.92 & 11.65 \\
\hline
\end{tabular}

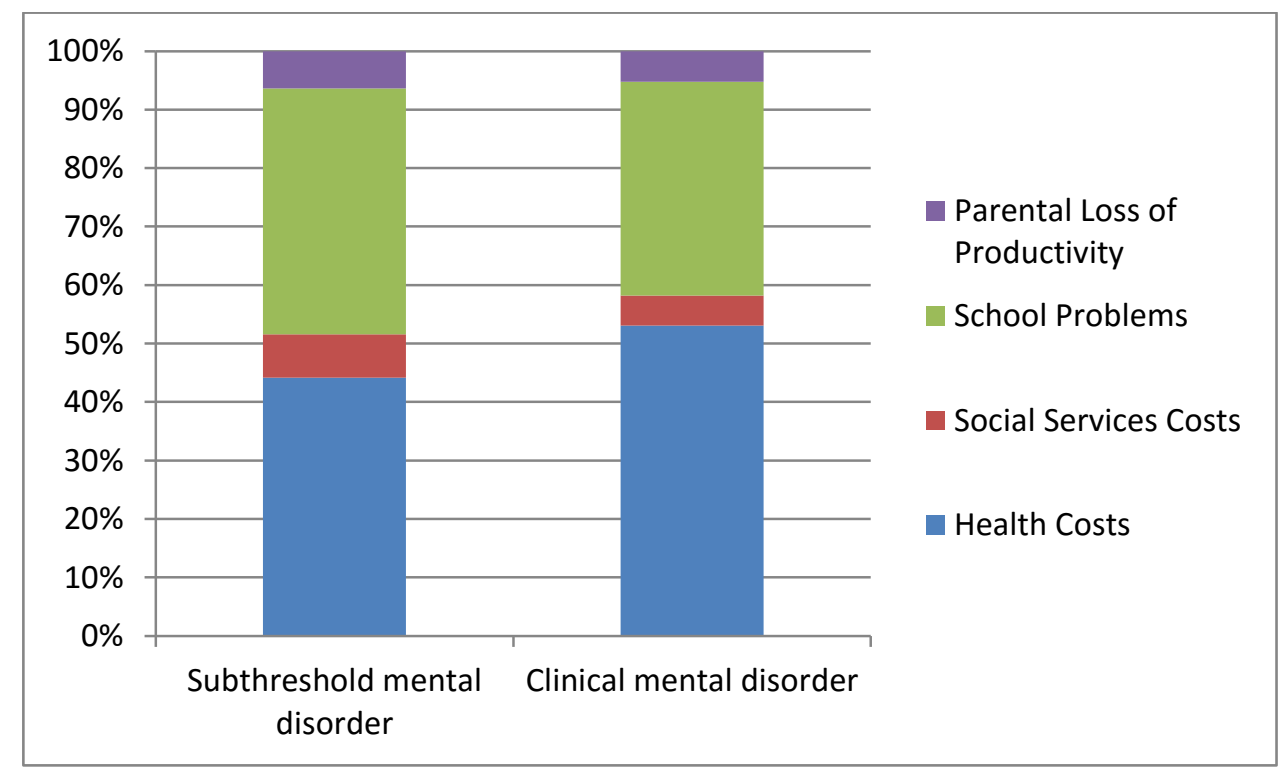

Figure 4. Distribution of the national cost estimate of childhood subthreshold and clinical mental disorders. 
ANEXO D. Artigo II submetido durante o período de doutoramento.

TÍTULO: Prevalência de Problemas de Saúde Mental na Infância na Atenção Primária

AUTORES: Daniel Fatori ${ }^{1}$, Alexandra Brentani ${ }^{2}$, Sandra Josefina Ferraz Ellero Grisi ${ }^{2}$, Euripedes Constantino Miguel ${ }^{1}$, Ana Soledade Graeff-Martins ${ }^{1}$,

${ }^{1}$ Instituto e Departamento de Psiquiatria do Hospital das Clínicas da Faculdade de Medicina da Universidade de São Paulo

${ }^{2}$ Instituto e Departamento de Pediatria do Hospital das Clínicas da Faculdade de Medicina da Universidade de São Paulo

Descritores: Atenção Primária à Saúde, Saúde Pública, Criança, Epidemiologia, Estudos Transversais, Brasil.

\section{INTRODUÇÃO}

O paradigma contemporâneo de saúde tem como peça fundamental e indissociável a saúde mental (1). O alto grau de limitação funcional e sofrimento causado pelos problemas de saúde mental resultam em grave impacto na qualidade de vida e produtividade. Estudo recente aponta que $22,7 \%$ dos anos vividos com incapacidade pela população mundial atualmente (years of life lost to disability; YLD) são decorrentes de problemas de saúde mental (PSM). A epidemiologia demonstra que os PSM frequentemente se iniciam na infância e adolescência (2), seguindo trajetória crônica (2).

No Brasil, estima-se que cerca de $13 \%$ da população de crianças e adolescentes tem algum diagnóstico de PSM $(3,4)$, com tendência dos sintomas persistirem de forma crônica. Dados brasileiros preliminares sugerem que entre casos graves e crônicos, apenas $37,5 \%$ tenham recebido algum tratamento num período de cinco anos (5). Essa alta taxa de prevalência, baixa taxa de tratamento, somada à trajetória crônica, torna a Atenção Primária (AP) do Sistema Único de Saúde (SUS) o âmbito ideal para 
prevenção, avaliação e tratamento dos PSM na infância e adolescência (6), já que é nesse contexto que a maioria da população brasileira busca atendimento em saúde (7).

A AP é um conjunto de iniciativas voltadas para a promoção da saúde, prevenção de agravos, tratamento e reabilitação. No Brasil é estruturada por meio da Estratégia da Saúde da Família (ESF), que tem como instrumento principal as equipes de saúde da família atuando nas Unidades Básicas de Saúde (UBS), configurando a porta de entrada do SUS. As equipes são compostas por um médico generalista, uma enfermeira, dois auxiliares de enfermagem e até seis agentes comunitários de saúde. Os profissionais das equipes de saúde da família têm como função avaliar, intervir e encaminhar para serviços especializados, quando necessário. Atualmente, a ESF cobre 62,5\% da população brasileira (8).

Apesar da relevância da ESF, há uma escassez de dados epidemiológicos e quantitativos sobre o perfil psicopatológico e o padrão de uso de serviços por crianças e adolescentes usuários dos serviços da AP do SUS. Dados dessas naturezas são essenciais para o planejamento adequado do sistema de saúde. Assim, o presente estudo tem como objetivo: (1) verificar a prevalência de PSM em uma amostra de crianças atendidas em UBSs da rede de AP de uma região do município de São Paulo; (2) descrever o uso de serviços de saúde nessa mesma amostra.

\section{MÉTODO}

Estudo populacional de corte transversal realizado em cinco UBSs da região oeste do município de São Paulo. O processo de amostragem se constituiu de um levantamento de todos os pacientes na faixa etária de 6-11 anos de idade que realizaram consultas (com atendimento registrado no Sistema Integrado de Gestão de Atendimento, SIGA) nessas UBSs nos meses de dezembro de 2011 a março de $2012(\mathrm{~N}=1423)$. As cinco UBSs mencionadas fizeram parte do projeto "Promoção da saúde mental na estratégia saúde da família: intervenção precoce visando à prevenção dos transtornos mentais e seu impacto socioeconômico", financiado pela Fundação de Amparo à Pesquisa do Estado de São Paulo (FAPESP), que capacitou profissionais de saúde no que tange a identificação e manejo de problemas de saúde mental na infância e adolescência (9). 
A coleta de dados foi realizada nos primeiros quatro meses de 2014. Os entrevistadores eram profissionais com ensino médio que receberam treinamento por pesquisador com experiência em estudos epidemiológicos. Os entrevistadores foram instruídos a entrarem em contato com as famílias por meio de visitas domiciliares. Nos casos em que as famílias não eram encontradas na primeira tentativa, os entrevistadores fizeram uma busca ativa no bairro/região. Todas as entrevistas aconteceram nos domicílios das famílias. Ao final deste processo foi possível localizar e entrevistar 825 pais/responsáveis das crianças (perda amostral: 42\%).

Os seguintes instrumentos de avaliação foram utilizados:

Versão adaptada do Client Service Receipt Inventory Children's version - questionário desenvolvido por pesquisadores do King's College London para levantar dados acerca do uso de serviços de saúde, tratamentos (incluindo medicamentoso e psicoterápico), serviços sociais e educacionais, entre outros (10), na infância e adolescência.

Strength and Difficulties Questionnaire (SDQ, Questionário de Capacidades e Dificuldades) - questionário que avalia crianças e adolescente na faixa etária de quatro a 16 anos e possui 25 itens distribuídos em cinco escalas: problemas emocionais, problemas de conduta, hiperatividade/déficit de atenção, problemas de relacionamento com colegas e comportamento social positivo, além da soma das primeiras quatro escalas, que representa o total de dificuldades (www.sdqinfo.com). A versão brasileira do SDQ se mostrou adequada para uso em estudo de validação (11) e tem sido utilizada em diversos estudos brasileiros (12). Os sujeitos de pesquisa foram categorizados por meio das escalas de problemas internalizantes (itens de problemas emocionais e problemas de relacionamento com colegas) e externalizantes (problemas de conduta e hiperatividade/déficit de atenção), cada uma com escore entre 0-20. O ponto de corte para categoria de nível clínico foi de $\geq 8$ pontos para internalizantes e $\geq 11$ pontos para externalizantes. Essa forma de categorização e respectivos pontos de corte se demonstraram adequados em estudo populacional prévio (13). 
O estudo teve aprovação da Comissão de Ética em Pesquisa da Faculdade de Medicina da Universidade de São Paulo. As entrevistas foram realizadas após o consentimento por escrito das mães/responsáveis, mantendo-se o sigilo das informações.

Foi realizada análise estatística descritiva das variáveis de interesse do estudo. Verificaram-se possíveis associações entre as variáveis por meio do teste de quiquadrado (teste exato de Fisher) e as odds ratio (OR; razão de chance) foram reportadas para todos os cruzamentos entre variáveis.

\section{RESULTADOS}

A amostra total foi de 825 crianças de 6-11 anos de idade (média: 8,23, desvio-padrão: 1,52), sendo que 49,9\% eram do sexo masculino. A distribuição de crianças da amostra quanto à cor de pele foi: branca $(57,6 \%)$, negra $(10,6 \%)$, entre branca e negra $(31,5 \%) \mathrm{e}$ indígena $(0,2 \%)$. A prevalência de PSM internalizantes (PSMI) e externalizantes (PSME) foi de 30,7\% e 18,3\%, respectivamente (ambos em nível clínico de acordo com o SDQ).

O pediatra foi o profissional de saúde mais consultado nos 12 meses antecedentes a entrevista com a mãe/responsável (56,7\%), seguido do médico de família $(47,6 \%)$ e clínico geral (19,0\%). Dentre os profissionais de saúde mental, o psicólogo foi o mais consultado $(7,9 \%)$, sendo que apenas $1,1 \%$ das crianças passaram por consulta com psiquiatra nos últimos 12 meses. Somente três crianças estavam em atendimento no CAPS. Nenhuma das 27 internações hospitalares nos últimos 12 meses aconteceram em razão de problema de saúde mental (tabela 1).

Apenas três crianças $(0,4 \%)$ estavam em tratamento medicamentoso para problemas de saúde mental nos últimos 12 meses. Os medicamentos psiquiátricos utilizados eram: valproato de sódio (sujeito 1), carbamazepina, fenorbital e diazepam (sujeito 2), cloridrato de clomipramina (sujeito 3). 
Tabela 1. Uso de serviços de saúde por crianças de 6-11 anos de idade nos últimos 12 meses $(\mathrm{N}=825)$.

\begin{tabular}{lc}
\hline & $\mathbf{N}(\mathbf{\%})$ \\
\hline Pediatra & $455(56,7 \%)$ \\
Clínico Geral & $149(19,0 \%)$ \\
Médico de família & $383(47,6 \%)$ \\
Neurologista & $25(3,1 \%)$ \\
Psiquiatra & $9(1,1 \%)$ \\
Psicólogo & $63(7,9 \%)$ \\
Psicopedagogo & $22(2,8 \%)$ \\
Fonoaudiólogo & $59(7,4 \%)$ \\
Assistente Social & $45(5,6 \%)$ \\
Terapeuta de família & $1(0,1 \%)$ \\
Terapeuta ocupacional & $8(1,0 \%)$ \\
CAPS & $3(0,4 \%)$ \\
Outros & $71(8,6 \%)$ \\
Internação hospitalar & $27(3,3 \%)$ \\
Qualquer serviço de SM & $78(9,8 \%)$ \\
\hline
\end{tabular}

Dentre crianças com PSM, o pediatra foi o profissional de saúde mais consultado nos últimos 12 meses (61,1\% e 56,4\%, entre crianças com PSMI e PSME, respectivamente). No mesmo período de tempo 53,2\% (PSMI) e 33,8\% (PSME) destas crianças passaram por consulta com algum profissional ou em serviço especializado em saúde mental (psicólogo, terapeuta ocupacional, terapeuta familiar, psiquiatra ou CAPS) (tabela 2).

Atendimentos nas seguintes especialidades nos últimos 12 meses demonstraram associação estatística com PSM (tabela 2): médico de família (PSMI: OR 1,4, p<0,05), psicólogo (PSMI: OR 2,4, p<0,001, PSME: OR 2,7, p<0,001), psicopedagogo (PSMI: OR 4,1, p<0,05), fonoaudiólogo (PSMI: OR 1,8, p<0.05), neurologista (PSMI: OR 4,2, $\mathrm{p}<0,001$ ), terapeuta ocupacional (PSME: OR 4,5, $\mathrm{p}<0,05$ ). Ter recebido atendimento com qualquer profissional ou em serviço especializado em saúde mental também se mostrou associado estatisticamente (PSMI: OR 2,8, p<0,001, PSME: OR 2,5, p<0,001). 
Tabela 2. Uso de serviços de saúde por crianças de 6-11 anos de idade nos últimos 12 meses por tipo de problema de saúde mental segundo o SDQ (N=825).

\begin{tabular}{ccccc}
\hline & \multicolumn{2}{c}{ Problemas Internalizantes } & \multicolumn{2}{c}{ Problemas Externalizantes } \\
\cline { 2 - 5 } & Sim & OR (IC 95\%) & Sim & OR (IC 95\%) \\
\hline Pediatra & $151(61,1 \%)$ & $1,3(0,9-1,8)$ & $84(56,4 \%)$ & $1,0(0,7-1,4)$ \\
Clínico Geral & $54(22,2 \%)$ & $1,3(0,9-2,0)$ & $31(21,1 \%)$ & $1,2(0,8-1,8)$ \\
Médico Família & $131(53,3 \%)$ & $1,4(1,0-1,9)^{*}$ & $77(52,4 \%)$ & $1,3(0,9-1,8)$ \\
Psicólogo & $33(13,3 \%)$ & $2,7(1,6-4,6)^{* *}$ & $22(14,9 \%)$ & $2,7(1,5-4,6)^{* *}$ \\
Psicopedagogo & $14(5,7 \%)$ & $4,1(1,7-9,9)^{*}$ & $6(4,2 \%)$ & $1,7(0,7-4,5)$ \\
Fonoaudiólogo & $25(10,2 \%)$ & $1,8(1,0-3,1)^{*}$ & $8(5,5 \%)$ & $0,7(0,3-1,5)$ \\
Assistente Social & $20(8,2 \%)$ & $1,9(1,0-3,4)$ & $14(9,6 \%)$ & $2,1(1,1-4,1)$ \\
Terapeuta familiar & 0 & $0,7(0,7-0,7)$ & 0 & $0,9(0,8-0,8)$ \\
Neurologista & $16(6,5 \%)$ & $4,2(1,8-9,6)^{* *}$ & $8(5,4 \%)$ & $2,1(0,9-5,1)$ \\
Terapeuta ocupacional & $4(1,6 \%)$ & $2,3(0,6-9,1)$ & $4(2,7 \%)$ & $4,5(1,1-18,3)^{*}$ \\
Psiquiatra & $5(2 \%)$ & $2,9(0,8-10,7)$ & $4(2,8 \%)$ & $3,7(1,0-13,8)$ \\
CAPS & $2(0,8 \%)$ & $4,5(0,4-50,2)$ & $2(1,4 \%)$ & $9,1(0,8-100,6)$ \\
\hline * $<0,05$ & & &
\end{tabular}

\section{DISCUSSÃO}

O presente estudo investigou a prevalência de PSM em crianças atendidas em UBSs da rede de atenção primária do município de São Paulo. A prevalência de PSM internalizantes e externalizantes foi de $30,7 \%$ e $18,3 \%$, respectivamente. As altas taxas encontradas se assemelham a estudos conduzidos em amostras comunitárias brasileiras. Por exemplo, um estudo em Ribeirão Preto (N=107) avaliou crianças de 6-11 anos de idade com o SDQ e encontrou as seguintes taxas: $30,8 \%$ de problemas emocionais, $14 \%$ de problemas interpessoais, $17,7 \%$ de problemas de conduta, 16,8\% de hiperatividade (14). Em Taubaté, também com o uso do SDQ em amostra comunitária, foi encontrada a taxa de $22,7 \%$ de total de problemas (nível clínico) em crianças de 7-11 anos ( $\mathrm{N}=454)$ (15). 
Até a década de 1980 a taxa de PSM em crianças e adolescentes na atenção primária era estimada em 4-15\% (16). Em 1988, um estudo nos Estados Unidos com 789 crianças de 7-11 anos encontrou uma taxa de $24,7 \%$ usando o Child Behavior Checklist (CBCL) (17). Nas décadas seguintes inúmeros estudos sobre o tema foram conduzidos.

Um estudo realizado nos Estados Unidos avaliou 21.065 crianças e adolescente de 4-15 anos, em toda a rede nacional de AP, e encontrou 13\% de PSM segundo o Pediatric Symptom Checklist (18). Em outro estudo norte-americano, com amostra de 767 crianças de 5-16 anos, 28\% apresentaram alta sintomatologia de acordo com o SDQ (19). Os dois estudos encontraram taxas de PSM inferiores aos dados da presente amostra.

Alguns estudos avaliaram especificamente PSMI em amostras de pacientes da AP. Um estudo nos Estados Unidos avaliou 714 crianças e adolescentes de 8-17 anos e encontrou $20 \%$ de sintomas de ansiedade segundo o instrumento Screen for Anxiety Related Emotional Disorders (20). Na França, pesquisadores avaliaram 155 crianças e adolescentes (7-17 anos de idade) que receberam atendimento na AP. Entre indivíduos com menos de 13 anos foi encontrada uma prevalência de depressão de $10 \%$ segundo o instrumento Center for Epidemiological Studies Depression (21). Estas duas pesquisas encontraram taxas significativamente menores do que a taxa de $30,7 \%$ de PSMI do presente estudo, apesar de também terem utilizado instrumentos de rastreamento.

Uma revisão sistemática verificou a prevalência de transtorno de déficit de atenção e hiperatividade (TDAH) na infância/adolescência em pacientes da AP e encontrou a taxa de 11,2\% na faixa etária de 6-12 anos de idade (22). Um grande estudo epidemiológico avaliou 22.059 crianças e adolescentes com 4-15 anos de idade nos Estados Unidos e Canadá, encontrando uma prevalência de TDAH de 9,2\% segundo avaliação clínica (23). A prevalência de PSME encontrada na amostra do presente estudo foi maior do que em ambos. Contudo, estes se utilizaram de diagnósticos segundo critérios do Diagnostic and Statistical Manual of Mental Disorders (DSM), tornando a avaliação mais específica e menos inclusiva. Além disso, PSME segundo o SDQ incluem problemas de conduta e hiperatividade, tornando a categoria mais ampla. 
No Brasil apenas um único estudo avaliou PSM em amostra de serviços da rede de atenção primária. Avaliando com o SDQ crianças de 5-11 anos que passaram por atendimento pediátrico em UBS, foi encontrado 55\% de casos de PSM (24), uma taxa muito mais alta que a encontrada no presente estudo. Contudo, os autores não descreveram como classificaram os casos de PSM, sendo possível terem incluído casos limítrofes na prevalência.

Os PSMI apresentaram associações com ter realizado atendimento nos últimos 12 meses com médico de família, psicólogo, psicopedagogo, fonoaudiólogo e neurologista, enquanto os PSME apresentaram associação com ter sido atendido por terapeuta ocupacional e psicólogo. A literatura internacional mostra que PSME costumam receber mais atenção em saúde do que PSMI (25). Logo, o dado encontrado pode significar uma diferença cultural no estilo parental, no sentido que sintomas de PSME talvez não sejam notados como prejuízo funcional ou um problema que necessita de atenção em saúde, enquanto sintomas de PSMI talvez levem a uma maior identificação da necessidade de atenção em saúde. Outra possibilidade é que os PSMI apresentem-se por meio de sintomas somáticos (como ocorre nos sintomas de ansiedade e depressão), levando os pais a procurarem ajuda. Considerando que professores podem apresentar boa capacidade de identificação de PSME em crianças após capacitação (26), sugere-se uma aproximação da escola com a rede de AP para uma melhor identificação de casos que necessitam de tratamento. Neste sentido, já existem programas efetivos disponíveis para professores escolares reconhecerem transtornos mentais na infância (27).

De forma geral, o perfil de uso de serviço na amostra apresentou pouco uso de atendimento psicológico $(7,9 \%)$ e psiquiátrico $(1,1 \%)$. Entre as crianças com PSM, $53,2 \%$ com PSMI e 33,8\% com PSME receberam algum tratamento especializado em saúde mental. Levando em conta a proposta da AP de avaliar e encaminhar casos que necessitam de atendimento especializado, ressalta-se o baixo número de crianças com PSM recebendo atendimento adequado, principalmente aquelas com PSME. Isso pode se dar em razão da baixa capacidade dos profissionais da AP em avaliar adequadamente casos que precisam de atenção especializada, ou à falta de profissionais especializados na $\mathrm{AP}$, ou ainda à dificuldade dos pais em perceber os sintomas de PSM como algo que necessita de tratamento. Também é possível que isso ocorra em função da carência de 
serviços especializados em saúde mental disponíveis, uma vez que a escassez de profissionais especialistas em saúde mental de crianças e adolescentes se verifica em todo mundo. Estudos brasileiros recentes, em diversas regiões do país, já demonstraram que entre 20\%-37\% das crianças e adolescentes com PSM recebem algum atendimento $(5,28)$.

O pediatra foi o profissional mais acessado na amostra (56,7\%), seguido do médico da família (47,6\%). Assim, pode ser interessante implantar programas de capacitação para que estes profissionais possam identificar e tratar os PSM mais comuns. Estudos têm demonstrado que programas de capacitação em saúde mental da infância para pediatras e para o médico dos programas de saúde da família são eficazes e trazem benefícios como mudança de atitude e melhor capacidade de encaminhamento (9). Como, entre os profissionais de saúde mental, o psicólogo se mostrou o mais procurado (28), especial atenção deve ser dada na formação destes profissionais para que estes possam realizar diagnósticos e intervenções baseadas em evidências e adequadas a esta faixa etária.

Nos últimos anos o tema do uso de medicamentos psiquiátricos na infância tem surgido na mídia $(29,30)$ e em artigos científicos (31). Contudo, as evidências apresentadas usualmente são inadequadas ou anedóticas, além de raramente pautadas em dados epidemiológicos. Numa amostra de 825 crianças atendidas na rede de AP foram encontradas apenas três crianças que fizeram uso de medicamentos psiquiátricos no último ano (e ainda assim, em pelo menos dois dos três casos, é possível que a criança estivesse usando medicamentos para o tratamento de doença neurológica), contrariando relatos que apontam prescrição de medicamentos psiquiátricos excessiva na infância. Assim, pelo contrário, a baixa prescrição de medicamentos psiquiátricos aliada a alta prevalência de PSM relatada neste artigo, reflete, possivelmente, a desassistência e falta de cuidados adequados a esta população. Um estudo prévio demonstrou que o transtorno de déficit de atenção e hiperatividade é subtratado no Brasil (32), corroborando com esta hipótese. Isto, aliado a evidências que demonstram que mais de $60 \%$ das crianças e adolescentes com transtornos mentais não recebem atendimento especializado em saúde mental $(5,28)$, revelam um cenário geral de desassistência. 
O presente estudo tem limitações. A perda amostral de $42 \%$ caracteriza um importante viés de seleção, uma vez que é impossível saber o que ocorreu com grande parte da amostra selecionada. Além disso, não foi possível comparar os sujeitos que não foram encontrados com a amostra final, pois as listas de pacientes obtidas não apresentavam informações sociodemográficas. Como a pesquisa se delimitou a uma região específica de apenas um município, não é possível generalizar os dados para demais regiões do município, muito menos para outras cidades e regiões do país. Por último, as avaliações dos sintomas de PSM foram conduzidas por meio de um instrumento de rastreamento (SDQ), não sendo possível determinar os critérios diagnósticos (segundo DSM) específicos de cada caso avaliado, nem definir se o caso é realmente positivo.

A alta prevalência de PSM em crianças atendidas na rede de AP se comparada a estudos internacionais e o baixo número de atendimentos em serviços especializados configuram um problema importante, principalmente se levarmos em conta que as UBSs estudadas encontram-se em região central da maior cidade do Brasil. Programas de capacitação para profissionais da AP, particularmente o pediatra e o médico da família, além de especial atenção a melhor qualificação do psicólogo, são importantes para melhorar a identificação de PSM na infância e, assim, aprimorar os encaminhamentos para serviços especializados. Quanto mais cedo os PSM na infância forem identificados e adequadamente tratados, melhor será o seu curso e prognóstico. Dessa forma, todo investimento em programas baseados em evidências focados nesta faixa etária configuram investimento da mais suma importância.

\section{AGRADECIMENTOS}

A Fundação de Amparo à Pesquisa do Estado de São Paulo ( $\mathrm{n}^{\mathrm{o}}$ do processo: 2009/53111-9), e aos profissionais da estratégia de saúde da família das unidades básicas de saúde estudadas.

\section{REFERÊNCIAS}

1. Prince M, Patel V, Saxena S, Maj M, Maselko J, Phillips MR, et al. No health without mental health. The Lancet. 2007 Sep;370(9590):859-77. 
2. Kim-Cohen J, Caspi A, Moffitt TE, Harrington H, Milne BJ, Poulton R. Prior juvenile diagnoses in adults with mental disorder: developmental follow-back of a prospective-longitudinal cohort. Arch Gen Psychiatry. 2003 Jul;60(7):709-17.

3. Paula CS, Coutinho ES, Mari JJ, Rohde LA, Miguel EC, Bordin IA, et al. Prevalence of psychiatric disorders among children and adolescents from four Brazilian regions. Rev Bras Psiquiatr. 2015 Jun;37(2):178-9.

4. Fleitlich-Bilyk B, Goodman R. Prevalence of child and adolescent psychiatric disorders in southeast Brazil. J Am Acad Child Adolesc Psychiatry. 2004 Jun;43(6):727-34.

5. Fatori D, Evans-Lacko S, de Paula C. Child mental health care in Brazil: barriers and achievements. The Lancet. 2012;379(9812):e16-7.

6. Lauridsen-Ribeiro E, Tanaka O. Organização de serviços no Sistema Único de Saúde para o cuidado de crianças e adolescentes com problemas de saúde mental. In: Atenção em Saúde Mental para Crianças e Adolescentes no SUS. Hucitec;

7. Paim J, Travassos C, Almeida C, Bahia L, Macinko J. The Brazilian health system: history, advances, and challenges. The Lancet. 2011 May;377(9779):1778-97.

8. Departamento de Atenção Básica. Histórico de Cobertura da Saúde da Família [Internet]. [cited 2015 Mar 27]. Available from: http://dab.saude.gov.br/portaldab/dab.php

9. Lowenthal, R. Capacitação em saúde mental na infância e adolescência para profissionais da atenção primária [Internet]. Universidade Presbiteriana Mackenzie; 2012. Available from: http://up.mackenzie.br/stricto-sensu/disturbiosdo-desenvolvimento/teses-e-dissertacoes-detalhada/artigo/capacitacao-em-saudemental-na-infancia-e-adolescencia-para-profissionais-da-atencao-primari/

10. Beecham J, Knapp M. Costing psychiatric interventions. In: Measuring Mental Health Needs [Internet]. Gaskell In Measuring Mental Health Needs. Edited by Thornicroft G. London; 2001 [cited 2014 Sep 1]. Available from: http://www.pssru.ac.uk/pdf/dp1536.pdf

11. Woerner W, Fleitlich-Bilyk B, Martinussen R, Fletcher J, Cucchiaro G, Dalgalarrondo P, et al. The Strengths and Difficulties Questionnaire overseas: evaluations and applications of the SDQ beyond Europe. Eur Child Adolesc Psychiatry. 2004;13 Suppl 2:II47-54.

12. Fleitlich B, Goodman R. Social factors associated with child mental health problems in Brazil: cross sectional survey. BMJ. 2001 Sep 15;323(7313):599600 .

13. Goodman A, Lamping DL, Ploubidis GB. When to Use Broader Internalising and Externalising Subscales Instead of the Hypothesised Five Subscales on the Strengths and Difficulties Questionnaire (SDQ): Data from British Parents, Teachers and Children. J Abnorm Child Psychol. 2010 Jul 10;38(8):1179-91. 
14. Cury CR, Golfeto JH. Strengths and difficulties questionnaire (SDQ): a study of school children in Ribeirão Preto. Rev Bras Psiquiatr São Paulo Braz 1999. 2003 Sep;25(3):139-45.

15. Vitolo YLC, Fleitlich-Bilyk B, Goodman R, Bordin IAS. Parental beliefs and child-rearing attitudes and mental health problems among schoolchildren. Rev Saúde Pública. 2005 Oct;39(5):716-24.

16. Costello EJ. Primary Care Pediatrics and Child Psychopathology: A Review of Diagnostic, Treatment, and Referral Practices. Pediatrics. 1986 Dec $1 ; 78(6): 1044-51$.

17. EJ C, AJ C, C E. Psychiatric disorders in pediatric primary care: Prevalence and risk factors. Arch Gen Psychiatry. 1988;45(12):1107-16.

18. Jellinek MS, Murphy JM, Little M, Pagano ME, Comer DM, Kelleher KJ. Use of the Pediatric Symptom Checklist to screen for psychosocial problems in pediatric primary care: a national feasibility study. Arch Pediatr Adolesc Med. 1999 Mar;153(3):254-60.

19. Brown JD, Wissow LS. Screening to identify mental health problems in pediatric primary care: Considerations for practice. Int J Psychiatry Med. 2010;40(1):1-19.

20. Chavira DA, Stein MB, Bailey K, Stein MT. Child anxiety in primary care: Prevalent but untreated. Depress Anxiety. 2004 Jan 1;20(4):155-64.

21. Mathet F, Martin-Guehl C, Maurice-Tison S, Bouvard M-P. [Prevalence of depressive disorders in children and adolescents attending primary care. A survey with the Aquitaine Sentinelle Network]. L'Encéphale. 2003 Oct;29(5):391-400.

22. Brown RT, Freeman WS, Perrin JM, Stein MT, Amler RW, Feldman HM, et al. Prevalence and assessment of attention-deficit/hyperactivity disorder in primary care settings. Pediatrics. 2001 Mar;107(3):E43.

23. Wasserman RC, Kelleher KJ, Bocian A, Baker A, Childs GE, Indacochea F, et al. Identification of attentional and hyperactivity problems in primary care: a report from pediatric research in office settings and the ambulatory sentinel practice network. Pediatrics. 1999 Mar;103(3):E38.

24. Tanaka OY, Lauridsen-Ribeiro E. A challenge for primary health care: mental health care implementation. Cad Saúde Pública. 2006 Sep;22(9):1845-53.

25. Costello EJ, Copeland W, Cowell A, Keeler G. Service costs of caring for adolescents with mental illness in a rural community, 1993-2000. Am J Psychiatry. 2007 Jan;164(1):36-42.

26. Vieira MA, Gadelha AA, Moriyama TS, Bressan RA, Bordin IA. Evaluating the effectiveness of a training program that builds teachers' capability to identify and appropriately refer middle and high school students with mental health problems in Brazil: an exploratory study. BMC Public Health. 2014 Feb 28;14(1):210. 
27. Pereira CA, Wen CL, Miguel EC, Polanczyk GV. A randomised controlled trial of a web-based educational program in child mental health for schoolteachers. Eur Child Adolesc Psychiatry. 2015 Aug;24(8):931-40.

28. Paula CS, Bordin IAS, Mari JJ, Velasque L, Rohde LA, Coutinho ESF. The Mental Health Care Gap among Children and Adolescents: Data from an Epidemiological Survey from Four Brazilian Regions. PLoS ONE. 2014 Feb 18;9(2):e88241.

29. Arraes J. Medicalização da infância: o TDAH e suas controvérsias [Internet]. Revista Fórum Semanal. [cited 2015 Apr 16]. Available from:

http://revistaforum.com.br/digital/171/medicalizacao-da-infancia-o-tdah-e-suascontroversias/

30. Brum E. O Doping das Crianças. Revista Época [Internet]. 2013 Feb 25 [cited 2015 Apr 3]; Available from: http://revistaepoca.globo.com/Sociedade/elianebrum/noticia/2013/02/o-doping-das-criancas.html

31. Decotelli KM, Bohre LCT, Bicalho PPG de. The drugs of obedience: medicalization, childhood and biopower: notes on clinic and bolitics. Psicol Ciênc E Prof. 2013 Jan;33(2):446-59.

32. Mattos P, Rohde LA, Polanczyk GV. ADHD is undertreated in Brazil. Rev Bras Psiquiatr. 2012 Dec;34(4):513-4. 
ANEXO E. Questões do protocolo de avaliação do estudo HRC relacionadas aos custos dos transtornos mentais na infância. 